University of South Florida

DIGITAL COMMONS

Digital Commons @ University of

@ UNIVERSITY OF SOUTH FLORIDA

South Florida

USF Tampa Graduate Theses and Dissertations

USF Graduate Theses and Dissertations

January 2015

\title{
Self-Assembly Kinetics of Microscale Components: A Parametric Evaluation
}

Jose Miguel Carballo

University of South Florida, jcarball@mail.usf.edu

Follow this and additional works at: https://digitalcommons.usf.edu/etd

Part of the Materials Science and Engineering Commons, and the Mechanical Engineering Commons

\section{Scholar Commons Citation}

Carballo, Jose Miguel, "Self-Assembly Kinetics of Microscale Components: A Parametric Evaluation" (2015). USF Tampa Graduate Theses and Dissertations.

https://digitalcommons.usf.edu/etd/5653

This Dissertation is brought to you for free and open access by the USF Graduate Theses and Dissertations at Digital Commons @ University of South Florida. It has been accepted for inclusion in USF Tampa Graduate Theses and Dissertations by an authorized administrator of Digital Commons @ University of South Florida. For more information, please contact digitalcommons@usf.edu. 
Self-Assembly Kinetics of Microscale Components:

A Parametric Evaluation

by

Jose M. Carballo

A dissertation submitted in partial fulfillment

of the requirements for the degree of

Doctor of Philosophy in Mechanical Engineering

Department of Mechanical Engineering

College of Engineering

University of South Florida

Major Professor: Nathan B. Crane, Ph.D.

Nathan Gallant, Ph.D.

George Nolas, Ph.D.

Vinay Gupta, Ph.D.

Rasim Guldiken, Ph.D.

Date of Approval:

June 30, 2015

Keywords: Electronics packaging, Microassembly, Performance evaluation, Modeling, Measurement, Surface tension

Copyright $@$ 2015, Jose M. Carballo 


\section{DEDICATION}

I would like to express my love and deepest appreciation for my parents, my brother, and future wife Alina. You have given me the courage, strength and unconditional support anybody could ask for. My diploma should have all your names. Now let's use it to keep our family moving forward. 


\section{ACKNOWLEDGMENTS}

While this work belongs to one author, definitely more than one person made it possible. I have many to thank for their contributions. First, I am forever grateful to Dr. Crane, my Ph.D. advisor who has been an outstanding and patient mentor since day 1 . His valuable lessons and undisputed expertise have provided guidance that not only pushed this project forward, but also

all my professional endeavors. Most importantly, Dr. Crane's high standards of quality provided me constant reason for improving myself.

I am also grateful to my graduate peers Ni Qi and Matt Trapuzzano for their immense support and valuable contributions to my project, and my learning. Also to my lab group peers at the Micro-Integration Laboratory: Dan, Justin and Clayton, who significantly helped expanding my knowledge beyond my project (I like to think so, at least). All my peers provided numerous intellectual conversations (and some rather cheerful) that made the work more enjoyable, and gratifying. I am also thankful to my undergraduate peers, Yohana, Jose Vasquez and Morgan for going the extra mile to help move the projects forward.

My committee members played an important role in my dissertation project evaluations. Their excellence and distinguished standing set a tough bar to reach, which I will always appreciate. Also, Dr. Jose Porteiro shared me with some of the most valuable guidance and wisdom that any graduate student can receive at the beginning of their Ph.D. program. Furthermore, Mr. Bernard Batson was constant support for keeping an eye out on countless opportunities for the near and long term future. 
Finally, many thanks to the staff and engineers at the USF Nanotechnology Research and Education Center. They have helped me jump major hurdles during my project tasks. Their jobs are to make mine easier, but they all have also gone above and beyond to provide infinite lessons that helped us become proficient, and competitive in micro/nano technology research. 


\section{TABLE OF CONTENTS}

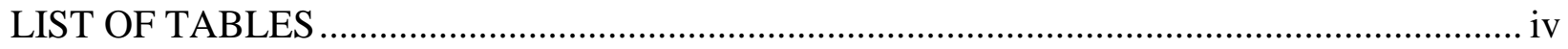

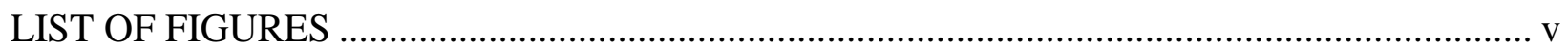

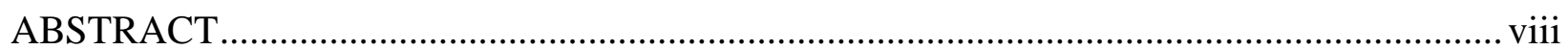

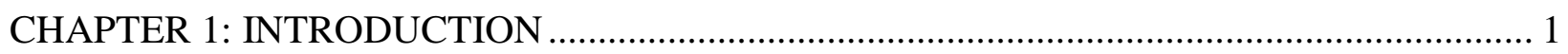

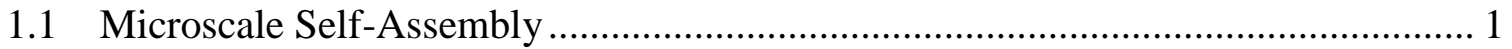

1.1.1 Limitations of Current Micro-Assembly Methods .................................... 2

1.1.2 Basics of a Stochastic SA Process .......................................................... 3

1.1.3 Advantages of SA at the Microscale......................................................... 5

1.1.4 Current Needs of SA: Easier Implementation for Microscale

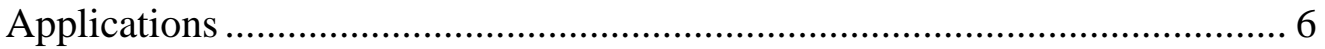

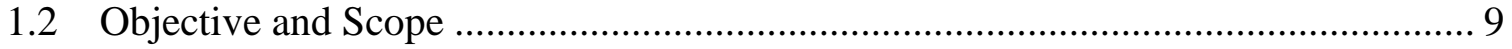

1.3 Dissertation Outline ................................................................................... 10

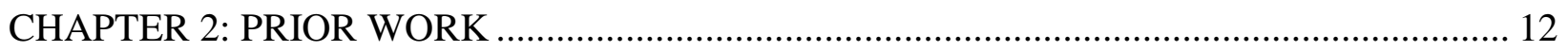

2.1 Successful Implementations of Microscale SA …………....................................... 12

2.1.1 Component-to-Substrate SA …………….......................................... 13

2.1.2 Component-to-Component SA ………………..................................... 16

2.1.3 Enhancing Capabilities of Microscale SA and its Applications ............... 17

2.2 Microscale SA Models: Motivation and Previous Work .......................................... 18

2.2.1 Bond Models …………………...................................................... 20

2.2.2 Process Models ....................................................................................... 23

2.3 Current Contribution to SA Process Models ……………………….................... 25

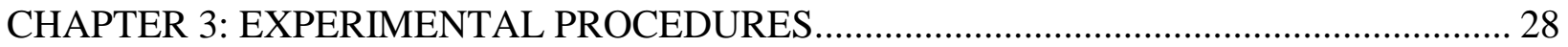

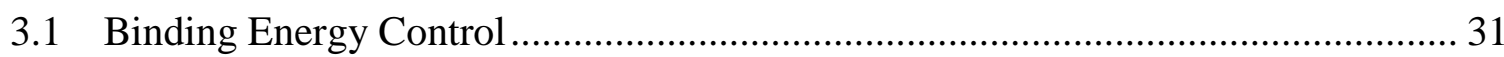

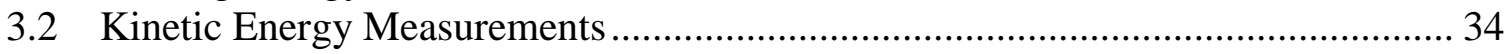

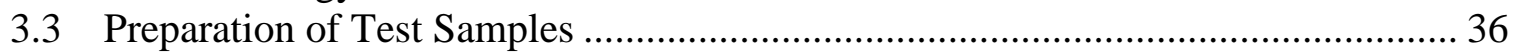

3.3.1 Water-Medium Samples .................................................................... 37

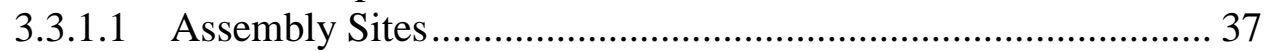

3.3.1.2 Assembly Parts........................................................................ 39

3.3.1.3 Surface Preparations ……………………………………...... 39

3.3.2 Air-Medium Samples........................................................................ 41

3.3.2.1 Assembly Sites................................................................... 41

3.3.2.2 Assembly Parts.................................................................... 43

3.3.2.3 Surface Preparations ............................................................... 43

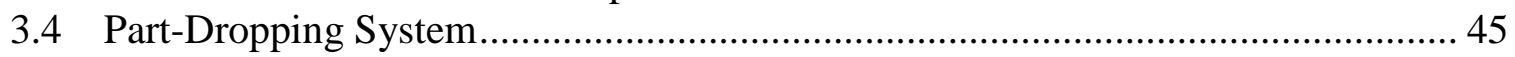


3.4.1 CNC Program for Pick-and-Drop Sequence ………................................. 45

3.4.2 Gripper Designs ............................................................................. 46

3.4.2.1 Capillary Gripper ................................................................. 47

3.4.2.2 Vacuum Gripper....................................................................... 48

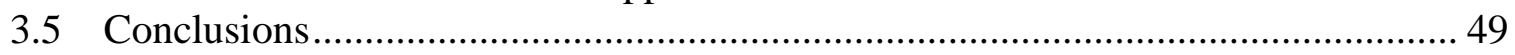

CHAPTER 4: SELF-ASSEMBLY KINETICS OF MICROSCALE COMPONENTS: A

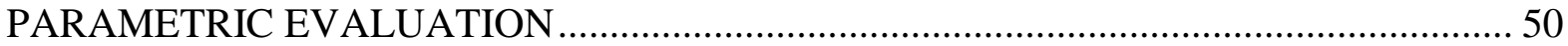

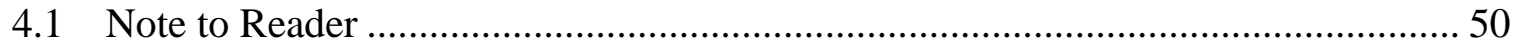

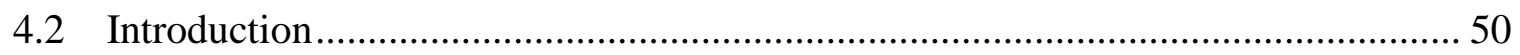

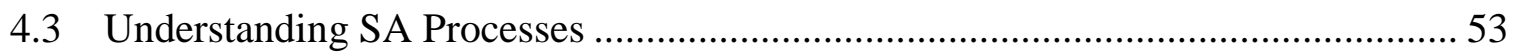

4.3.1 Experimental SA System .................................................................... 57

4.3.1.1 Experimental Procedure.......................................................... 57

4.3.1.2 Controlling SA Parameters ........................................................ 60

4.3.1.2.1 Kinetic Energy ........................................................... 60

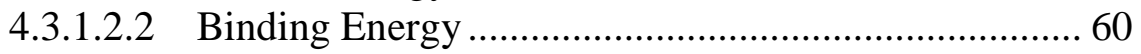

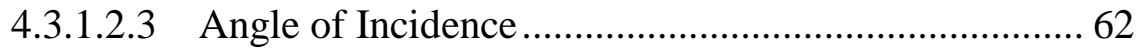

4.3.1.2.4 Orientation Probability ................................................. 62

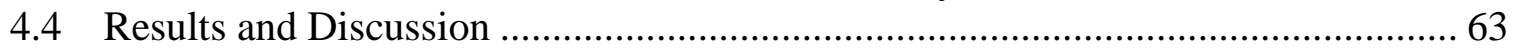

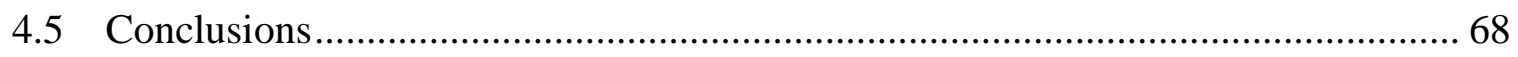

CHAPTER 5: MONTE CARLO STUDY OF SELF-ASSEMBLY DATA VARIATION........... 71

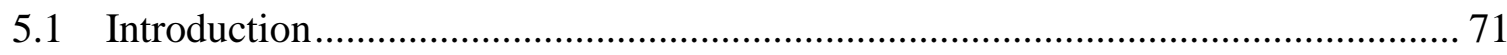

5.2 Monte Carlo Simulation Procedure ……………….............................................. 72

5.2.1 Method for Simulating Landing Locations............................................. 73

5.2.2 Method for Measuring Inaccuracy of Pick-and-Drop System................... 74

5.2.3 General Procedure for Monte Carlo Simulations........................................ 76

5.3 Monte Carlo Simulations of Part-Site Interactions ................................................. 79

5.4 Monte Carlo Simulations of Assembly Rate …………........................................ 80

5.5 Analysis of Optimum Strategy for SA Tests …………………………………..... 85

5.5.1 Linear Approximation of $1^{\text {st }}$ Order Kinetics Reaction............................... 85

5.5.2 Revised Strategy for Assembly Rate Measurements ................................ 88

5.5.3 Analysis of Results .............................................................................. 89

5.5.3.1 Implementing New Strategy to Future SA Experiments ........... 98

5.6 Conclusions .................................................................................................. 99

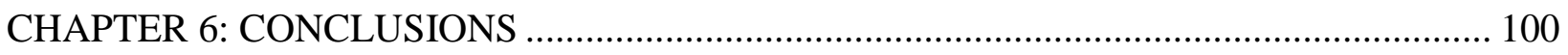

6.1 Key Conclusions ............................................................................................ 102

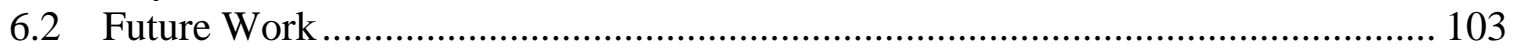

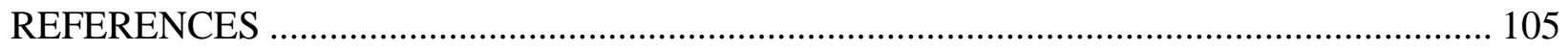

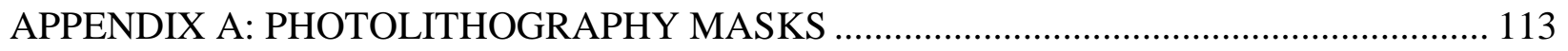

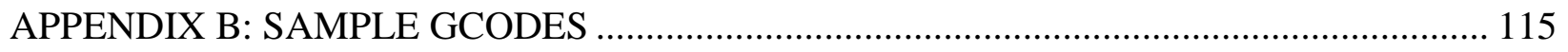

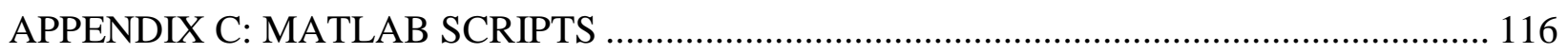


C.1 Matlab Script for Image Analysis and Part-Location Recognition.

C.2 Matlab Script for Generating GCode Programs that Run the Pick-and-Drop

System During SA Tests

APPENDIX D: COPYRIGHT PERMISSION 


\section{LIST OF TABLES}

Table 1: Contact angle measurements for determination of binding energy $\left(E_{b}\right)$ for possible contact types. ........................................................................... 34

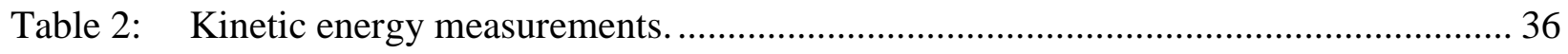

Table 3: Assembly site height measurements for air-medium tests................................... 44

Table 4: Summary of drop accuracy measurements. .................................................. 76

Table 5: Comparison of assembly rate variation between water-medium experiments

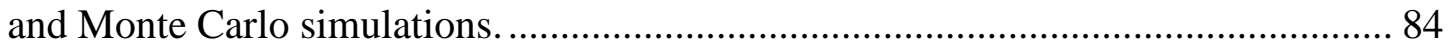

Table 6: Parameters for simulations considering water-medium experiment conditions, and dropping over 1 strip at a time. ........................................................... 90

Table 7: Results of Monte Carlo simulation for different drop areas. .................................. 95

Table 8: Results of Monte Carlo simulation for different misalignment tolerances................ 98 


\section{LIST OF FIGURES}

Figure 1: Plot of assembly rate vs. component size for traditional assembly methods.............. 5

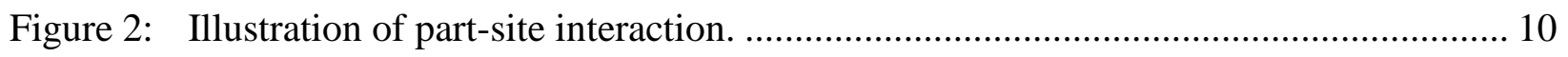

Figure 3: Hydrophobic bonding between a part and an assembly site ................................ 14

Figure 4: Effects of bond geometry on minimum energy states. ..................................... 21

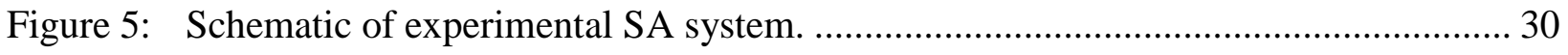

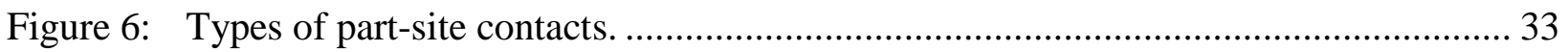

Figure 7: System for measuring part-velocity ........................................................... 35

Figure 8: Geometry of substrates with assembly sites used for studying $\varphi$ and $\rho_{o}$

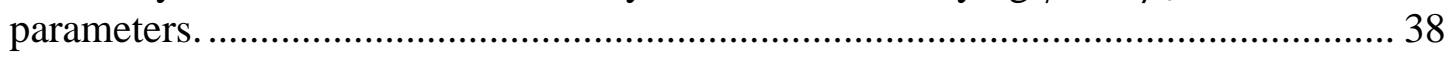

Figure 9: Steps for fabricating water medium assembly sites........................................... 39

Figure 10: Illustration of pass-through interface procedure for selective oil-coating of

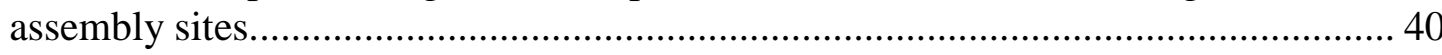

Figure 11: Experimental system for air-medium tests. ................................................... 41

Figure 12: Procedure for fabricating air-medium assembly sites.................................... 42

Figure 13: Geometry and mounting fixture of air-medium assembly sites........................... 43

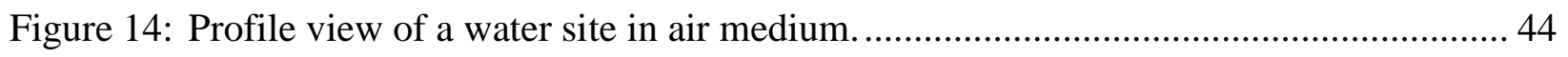

Figure 15: (a) Capillary gripper (a) and vacuum gripper (b) ........................................... 47

Figure 16: Flow chart of vacuum gripper actuation process (a) and vacuum gripper

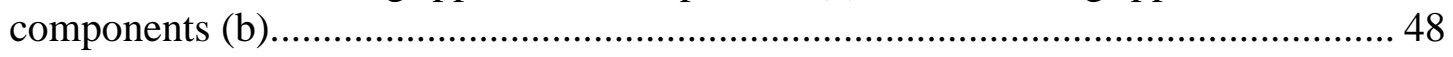

Figure 17: Unit cell of a self-assembly system consists of a single site................................ 55

Figure 18: (a) Process for preparing assembly sites ........................................................ 58

Figure 19: Sample chart showing assembly increments during one SA test...........................59 
Figure 20: Surface compositions of binding and non-binding surfaces in assembly parts and sites.

Figure 21: Illustration of each part geometry used for SA tests, and the measured orientation probability $\left(\rho_{o}\right)$ values.

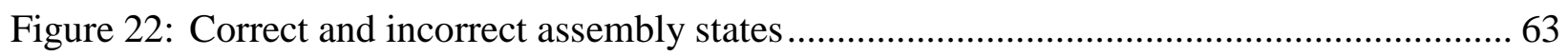

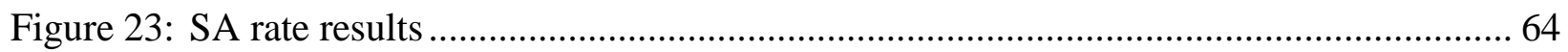

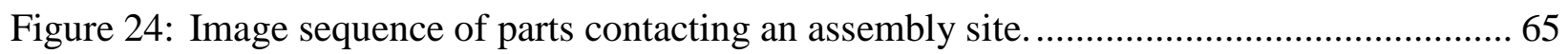

Figure 25: (a) Different levels of hypothesized misalignments are illustrated, arranged in order from minimum to maximum possible misalignment (from left to right).

Figure 26: Assembly sites SA rate vs. $\rho_{o}$ were measured for assembly sites with varied overlaying space.

Figure 27: Sample image captured for measuring drop accuracy of the pick-drop system. 75

Figure 28: Illustration of boundaries for drop area and assembly sites. 77

Figure 29: Configurations of assembly site geometries, as implemented in Monte Carlo simulations of water-medium experiments. 78

Figure 30: Progress of on-site landings for incremented number of parts dropped. 80

Figure 31: Results from 3 simulation trials, displaying the change in assembled sites $\left(B / A_{o}\right)$ with the increasing number of parts dropped. 82

Figure 32: Results from Monte Carlo simulations considering water-medium test conditions. 83

Figure 33: Histogram plots of assembly rate data from Monte Carlo simulations replicating water-medium testing conditions.

Figure 34: General curve of the integrated law of a $1^{\text {st }}$ order reaction. 86

Figure 35: Comparison between regression fitting, and linear approximation for acquiring assembly rate data from Monte Carlo simulations.

Figure 36: (a) Results from Monte Carlo simulations considering water-medium test conditions $(\sigma=6 \mathrm{~mm})$, and revised strategy (single strip instead of 12 strips).

Figure 37: Geometry for drop area and assembly site boundaries, implemented on $2^{\text {nd }}$ set (a) and $3^{\text {rd }}$ set (b) of Monte Carlo simulations for the study of assembly rate variation. 
Figure 38: Progress of assembly rate as the number of parts dropped are incremented, for different drop area simulations................................................................. 94

Figure 39: Distribution of part landing locations for drop areas of different sizes.................. 97

Figure A.1: Photolithography mask for water-medium test sites...................................... 113

Figure A.2: Photolithography mask for air-medium test sites ....................................... 114

Figure B.1: Sample GCode program for running CNC-driven pick-and-drop system ............ 115 


\begin{abstract}
The goal of the present work is to develop, and evaluate a parametric model of a basic microscale Self-Assembly (SA) interaction that provides scaling predictions of process rates as a function of key process variables. At the microscale, assembly by "grasp and release" is generally challenging. Recent research efforts have proposed adapting nanoscale self-assembly (SA) processes to the microscale. SA offers the potential for reduced equipment cost and increased throughput by harnessing attractive forces (most commonly, capillary) to spontaneously assemble components. However, there are challenges for implementing microscale SA as a commercial process. The existing lack of design tools prevents simple process optimization. Previous efforts have characterized a specific aspect of the SA process. However, the existing microscale SA models do not characterize the inter-component interactions. All existing models have simplified the outcome of SA interactions as an experimentally-derived value specific to a particular configuration, instead of evaluating it outcome as a function of component level parameters (such as speed, geometry, bonding energy and direction). The present study parameterizes the outcome of interactions, and evaluates the effect of key parameters. The present work closes the gap between existing microscale SA models to add a key piece towards a complete design tool for general microscale SA process modeling.
\end{abstract}

First, this work proposes a simple model for defining the probability of assembly of basic SA interactions. A basic SA interaction is defined as the event where a single part arrives on an assembly site. The model describes the probability of assembly as a function of kinetic energy, 
binding energy, orientation and incidence angle for the component and the assembly site. Secondly, an experimental SA system was designed, and implemented to create individual SA interactions while controlling process parameters independently. SA experiments measured the outcome of SA interactions, while studying the independent effects of each parameter.

As a first step towards a complete scaling model, experiments were performed to evaluate the effects of part geometry and part travel direction under low kinetic energy conditions. Experimental results show minimal dependence of assembly yield on the incidence angle of the parts, and significant effects induced by changes in part geometry. The results from this work indicate that SA could be modeled as an energy-based process due to the small path dependence effects. Assembly probability is linearly related to the orientation probability. The proportionality constant is based on the area fraction of the sites with an amplification factor. This amplification factor accounts for the ability of capillary forces to align parts with only very small areas of contact when they have a low kinetic energy. Results provide unprecedented insight about SA interactions. The present study is a key step towards completing a basic model of a general SA process. Moreover, the outcome from this work can complement existing SA process models, in order to create a complete design tool for microscale SA systems.

In addition to SA experiments, Monte Carlo simulations of experimental part-site interactions were conducted. This study confirmed that a major contributor to experimental variation is the stochastic nature of experimental SA interactions and the limited sample size of the experiments. Furthermore, the simulations serve as a tool for defining an optimum sampling strategy to minimize the uncertainty in future SA experiments. 


\section{CHAPTER 1: \\ INTRODUCTION}

\subsection{Microscale Self-Assembly}

Microscale devices are commonly fabricated on substrates of macroscale or mesoscale size $(<1 \mathrm{~mm})$. For such cases, standard (macro) manipulation tools transport the substrates into a final product package. However, other designs require integrating micro-scale components produced separately. Micro-integration is essential for components coming from incompatible fabrication processes and/or incompatible materials. Hybrid processing, for example, integrates micro-components (such as electronic chips, sensors and actuators) commonly fabricated with micro-electronics techniques with products coming from standard macro-fabrication processes (machining, forming, molding, etc.) (Hofmann, 2010). In other cases, micro-components may come from different microscale technologies, such as complementary metal oxide semiconductor (CMOS) and microelectromechanical systems (MEMS) processes. Each of these processes deserve their own production line in order to preserve feasibility and efficiency (Cohn et al., 1998). In all these cases, microscale components are first separated from their original substrate. Then micro-assembly is required for integrating these components another substrate, or device.

Micro-manipulation tools are expensive, and can only work in a serial fashion.

Consequently, "pick-and-place" micro-assembly methods encounter significant cost, and process rate challenges. The present work focuses on a novel approach for micro- assembly, called SelfAssembly (SA). This approach already exists (most commonly) in nature, and nanoscale manufacturing. The present chapter explains the expected advantages that SA offers over current 
micro-assembly technology. However, industrial implementation of a microscale SA process is challenging and there are very limited models available to guide process development. This chapter also provides background for understanding what these challenges are, and how can they be addressed. Subsequently, this chapter describes the goals for the proposed a process model for microscale self-assembly that is evaluated in this thesis. This model will serve as a process design tool; hence facilitate the implementation of a microscale SA process, with optimum performance.

\subsubsection{Limitations of Current Micro-Assembly Methods}

Current technologies for micro-device assembly are commonly serial, "pick and place" methods - typically utilizing either vacuum or mechanical gripping for manipulation (Carlisle, 2009). These serial-based manipulation systems have been developed to achieve assembly rates peaking at >1,000 parts/hour (C.J. Morris, Stauth, \& Parviz, 2005). However, significant challenges arise when adapting such assembly methods to microscale assembly.

Naturally, microscale components (part sizes between $10^{-8} \mathrm{~m}$ and $10^{-5} \mathrm{~m}$ ) require motion and manipulation systems with higher precision. These design requirements reach (even exceed) the precision limits of standard macroscale systems (Chen \& Sun, 2013). Furthermore, mechanical and vacuum tools are much larger than the assembly components. While it may still be possible to use such mechanisms for microscale components, closed loop control is generally required. Feedback mechanisms encounter significant challenges at the microscale. For instance, visual feedback (the most common feedback type) requires expensive optics to image microscale components with high magnification. Moreover, the size of high magnification optics imposes a limiting constraint on the design of a manipulation system. On the other hand, less expensive (i.e. lower magnification) optics provide limited position precision, and almost none information 
about component-orientation. Consequently, microscale manipulation systems involve greater system costs for achieving high assembly precision.

More importantly, at the microscale, inertia forces become relatively insignificant, while surface tension, Van der Waals and electrostatic forces dominate. While this may not impose a challenge for grasping parts, the release step becomes more difficult. For instance, after grasping a component, it may stick to the tool surfaces even when the vacuum gripper is disengaged, or the mechanical gripper is opened. Hence, a release force is also needed, such as positive pressure, or vibrations (Fearing, 1995). It is likely that such energy applications will cause alignment precision to decrease (Carlisle, 2009).

SA offers advantages that circumvent the afore-mention challenges of traditional microassembly methods. However, SA also encounters a different set of limitations, which are addressed by the present study. The following section provides first a brief explanation of an SA process, and then a description of the main advantages and limitations of SA as a microassembly method.

\subsubsection{Basics of a Stochastic SA Process}

As the name suggests, self-assembly (SA) is the process during which components spontaneously assemble. Energy minimization drives the components into an assembled state, instead of being driven by a manipulation tool. Such concept exists in nature, most commonly at the molecular scale (Whitesides \& Grzybowski, 2002). The process of molecular assembly is a stochastic self-assembly process. Molecules interact stochastically until favorable conditions are met. At this point, the components assemble. The final self-assembled structure represents a balance between the formation of bonds that reduce energy and the action of any disruptions (thermal vibrations at the nanoscale) that would break the bonds. 
At any scale, a stochastic self-assembly process involves three basic steps: transport, alignment and bonding. In the first step, assembly components are transported by a certain force field towards their assembly location. Generally, components are transported along random motion paths. Secondly, once a component encounters an assembly site (during its random motion), it "interacts" with the site through the bonding force. This force (within some range) will act to align the components into the assembly position. This step occurs because the assembly position corresponds to a state of minimum energy. Finally, the component achieves an energy minimum and remains bonded to the site. During the final phase, the component resists disruptive forces that would otherwise break the bond (Pelesko, 2007).

There are infinite examples of SA in nature. Common examples from the macroscale are in the "Cheerios effect" (floating cereal pieces in a breakfast bowl assembling into a raft) (Pelesko, 2007), and in the crystalline structure that spheres form when enclosed in a container. For the case of molecular SA, the alignment and bonding steps could be driven by Van der Waals, electrostatic or hydrogen bonds. Furthermore, SA processes have been engineered for many nanoscale applications; e.g. chemical synthesis and DNA assembly (Pelesko, 2007). These nanoscale examples are very well understood, given the amount of research dedicated to the corresponding mechanisms.

On the other hand, microscale SA is far less common in nature, and more difficult to engineer. At the microscale, stochastic interactions occur at a much slower rate. Generally, components need to travel longer distances, and do so at much slower speeds (Nathan B. Crane, Onen, Carballo, Ni, \& Guldiken, 2013; Pelesko, 2007; Whitesides \& Grzybowski, 2002). Nevertheless, microscale SA has recently been demonstrated to provide strong advantages; 
specifically for assembling microscale devices. These advantages are covered in the following section.

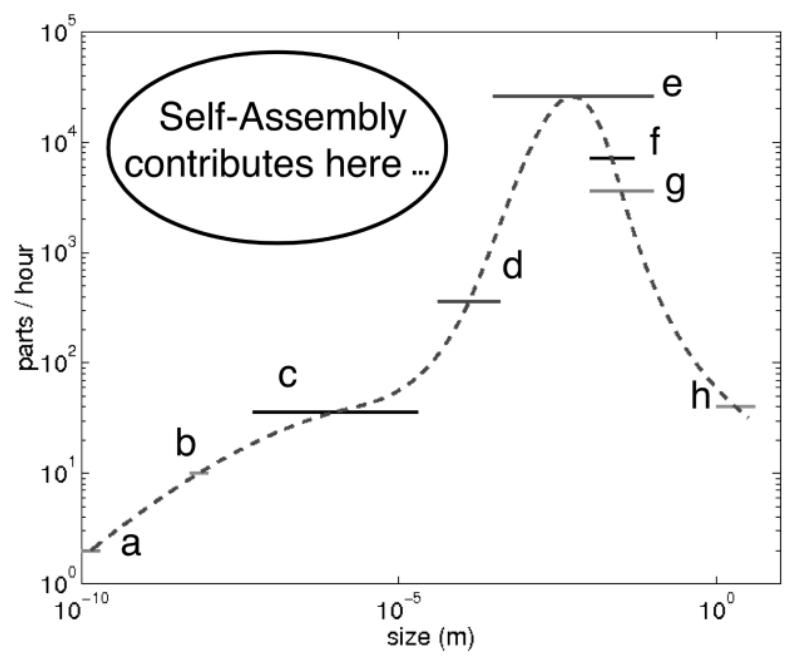

Figure 1 Plot of assembly rate vs. component size for traditional assembly methods. As size of assembly components decreases the current "pick-and-place" methods become more efficient and more effective (likely due to the reduction in mass inertia). As sizes reduce, the ability to manipulate components becomes challenged by precision requirements. Moreover, when component sizes reach the millimeter and micrometer range, surface tension, Van der Waals and electrostatic forces become increasingly dominant. These impose significant reductions in rate for any "pick and place" mechanism (C.J. Morris et al., 2005). (@ 2005 IEEE)

\subsubsection{Advantages of SA at the Microscale}

Besides pushing the limits of macroscale methods, different solutions have been implemented for achieving micro-assembly. Some very comprehensive reviews about micromanipulation systems have been published recently (Carlisle, 2009; Chen \& Sun, 2013; Cohn et al., 1998; Gauthier \& Regnier, 2010). However, grasp-release -processes do not scale as part sizes decrease further (C.J. Morris et al., 2005) . Figure 1 published by Morris et al illustrates how serial-based assembly rates change with respect to part size scale.

This figure illustrates the main advantage that microscale SA would have over current serial methods (part sizes between $10^{-8} \mathrm{~m}$ and $10^{5} \mathrm{~m}$ ). Being a parallel process, SA has the potential for offering much higher throughput. Additionally, SA does not require micro- 
manipulation tools. Instead, SA harnesses force fields that achieve minimum energy states for the desired assembly position (and orientation). Therefore, SA eliminates the need for expensive manipulation systems, thus reduces overall system cost. Morris et al also reviewed SA systems that have successfully demonstrated these advantages (C.J. Morris et al., 2005). Moreover, Chapter 2 gives a more focused review of successful SA implementations.

SA also has potential to complement existing additive manufacturing techniques for the purpose of integrating components within the product. On one hand, microscale SA could be considered an additive manufacturing process on its own when self-assembling (microscale) building blocks together, or on a substrate. For this scenario, SA would offer the unique capability of creating functional 3D structures (Breen, 1999; Terfort, Bowden, \& Whitesides, 1997). On the other hand, embedding microscale components during an additive manufacturing process could be approached with SA. Embedded components enhance functionality of an additive manufacturing product (Kataria \& Rosen, 2001). Crane et al discuss the implications on product performance, from embedding components through SA (N.B. Crane, Tuckerman, \& Nielson, 2011). While this is a promising approach for embedding microscale components, there are challenges that currently prevent its implementation.

\subsubsection{Current Needs of SA: Easier Implementation for Microscale Applications}

While SA has extensively demonstrated its potential advantages as a micro-assembly process, there are yet important challenges that hinder its implementation in mass-production. One main obstacle is that process requirements of microscale SA are dramatically more constraining than those of grasp and release methods. In other words, component geometry and material composition need to be defined around the requirements for designing SA bonds (Crane et al. 2013). On the other hand, some grasp and release methods (such as vacuum and 
mechanical grippers) do not (generally) impose as much constraint on the component design (Gauthier and Regnier 2010).

Additionally, previous demonstrations of SA systems have been successful for specific applications and conditions. Much experimental work has been published in the area of microscale SA. Nevertheless, these works have not provided guidance for general design of SA systems. In order to facilitate implementation of SA, design tools are needed.

As a general design objective of a SA process, process yield and process rate shall be maximized. With SA being a stochastic process, the objective of maximum process rate can be broken down further into two strategies: 1) maximizing the probability of assembly for each interaction; and 2) maximizing the rate at which stochastic interactions occur. On the other hand, maximizing process yield involves -among other requirements -optimization of assembly accuracy, and minimization of the probability for incorrect assemblies (bond at undesired locations or orientations, causing a defect in the final product). Incorrect assemblies compromise performance of the assembled device. Moreover, SA configurations possess a certain misalignment tolerance, above which device performance is unacceptable.

Modeling efforts have focused on different aspects of an SA process, while targeting one of the two design objectives (maximize assembly probability and maximize rate of interactions). Most SA models have focused on the design of the assembly bonds, for parameters such as bonding force (K. F. Bohringer, Srinivasan, \& Howe, 2001; Greiner et al., 2002; Lu, Xia, Liu, \& Zhang, 2006; Ramadoss \& Crane, 2008; Xiaorong Xiong, Liang, \& Bohringer, 2004; Zhang et al., 2005) and bond selectivity (Onoe, Matsumoto, \& Shimoyama, 2004; Srinivasan, Liepmann, \& Howe, 2001; Xiaorong Xiong et al., 2004). These bond models focus on the force, stiffness, and/or local minima in the bonding. These bond models can be used to verify that the parts can 
assemble, but the models do not provide direct information on the assembly rates or probabilities. Typically, the bonds forces are strengthened in order to increase the assembly rate and local minima in the bonding forces are eliminated to reduce errors. However, the relationship between bonding strength and assembly rate is not understood. Hence, these models assure that the assembly probability is nonzero (objective 1). However, bond models do not offer a solution for maximizing process rate. In fact, bond models do not consider other interacting effects, such as kinetic energy, direction, orientation and amount of components.

On the other hand, other SA models focused on optimizing process parameters in order to increase the rate of interactions (objective 2). For instance, such models have studied the effects on process rate, from varying the relative amount of components and receptor sites (W. Zheng \& Jacobs, 2005), the possible intermediate assembly states (Hosokawa, Shimoyama, \& Miura, 1994), agitation energy and misalignment tolerance (Mermoud, Brugger, \& Martinoli, 2009). The latter parameter can be also represented by assembly probability. However, modeling efforts had either chosen an arbitrary value for this parameter, or previously measured it (for specific scenarios).

Besides bond models, there is a lack of guidance for defining, and maximizing assembly probability. The main hurdle for achieving this goal is in the lack of relations that define the physics of assembly interactions. In other words, the following questions remain: what are all the parameters that control such interactions? How can we tune such parameters for maximum assembly probability? More importantly, these questions shall be answered for a general SA process, rather than just for specific applications. A more detailed review of the SA modeling works is provided in Chapter 2, as well as more expanded analysis for supporting the present conclusion. 


\subsection{Objective and Scope}

The present work addresses the need for a parametric understanding of SA process rates. For this purpose, an experimental study of basic SA interactions is provided. First, this study is focused on the most basic of SA interactions: one part directly landing on one assembly site. Figure 2 illustrates such an event. This is a representative unit-cell of a component-to-substrate SA process. In an actual SA process, assemblies could also occur from more complex interactions, such as multiple parts interacting with one site, or one part bouncing off nonbinding surfaces onto a site. While, this study is not focused on characterizing such complex interactions, most complex interactions can be broken into a simpler interaction in terms of the process variables studied here. For example, a site assembling after bouncing off another object is the same as a direct assembly except with potential change in different arrival angle, orientation, and velocity. The goal of this project is to provide initial understanding of the basic assembly event, which could then be expanded towards more complex studies.

Hendrick proposed a hypothetical parametric model (Hendrick, 2010) was developed to capture the outcomes of this basic SA interaction. Furthermore, key assumptions were defined to allow independent evaluation of each parameter. The present study focuses on the empirical evaluation of these parameters. For this purpose, an experimental system was designed. Such system allowed independent control of process parameters for every SA interaction. SA experiments consisted of creating a large number of individual part-site interactions, and measuring statistics of their outcome

The experimental relationships allowed us to test the proposed parametric model of SA interactions. This resultant model is expected to become an important contribution towards 
building a complete tool for SA process design; hence, towards facilitating the implementation of SA in micro-assembly applications.

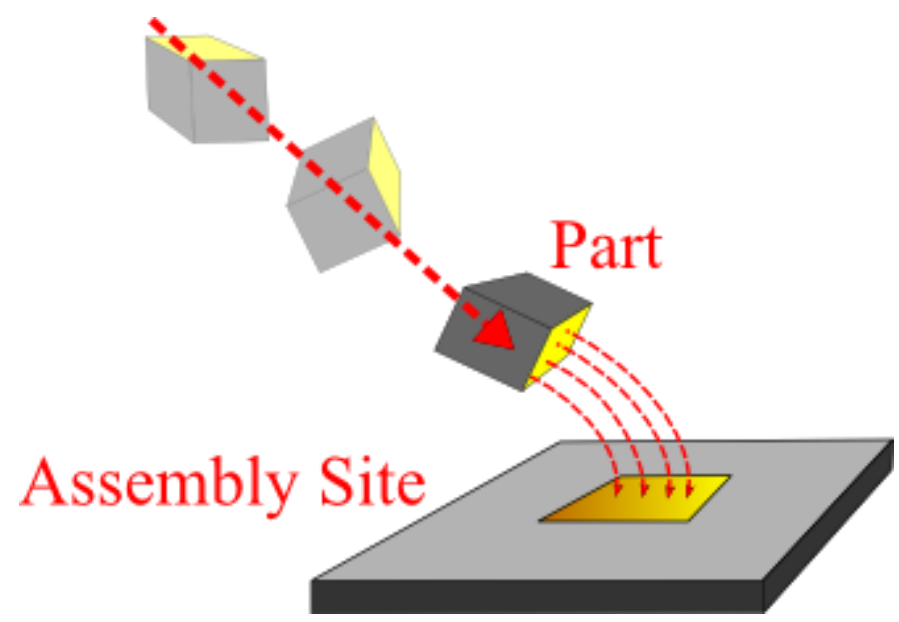

Figure 2 Illustration of part-site interaction. A basic SA interaction involves one part landing directly on one assembly site. This is a representative unit cell of a component-to-substrate SA process. More complex interactions fall outside of scope for this study.

\subsection{Dissertation Outline}

The following chapters start by providing the context of where the present work fits in the overall picture of microscale SA. Chapter 2 reviews the successful demonstrations that implemented SA for micro-assembly. Subsequently, a focused review of the modeling works helps the reader understand the motivation behind the present study.

Next, Chapter 3 provides details of the experimental SA system that was developed for evaluating SA interactions. In order to run SA experiments, environmental conditions and experimental variables were controlled. This chapter depicts the procedure for measurements that demonstrate successful control of such variables and conditions. Chapter 4 contains the first set of SA tests, which evaluate whether the direction of part-motion relative to the site affects the assembly probability.

The experimental results in Chapter 4 possessed significant amount of noise. Monte Carlo simulations confirmed that experimental variation was mostly due to low sampling. 
Moreover, conclusions from simulations provide a new sampling strategy with lower inherent noise. Chapter 5 provides details of this Monte Carlo study.

Lastly, Chapter 6 compiles the overall conclusions of the present work. Additionally, conclusions are related to the contributions that this work provides. Lastly, recommendations for future work are provided, with the interest of achieving the goals above stated. 


\section{CHAPTER 2:}

\section{PRIOR WORK}

This chapter addresses a general summary of successful micro-SA implementations, and what the challenges are for successful large scale implementation of SA. This summary covers the most significant works that have implemented the concept of self-assembly (SA) for micrometer and millimeter scale components. Such works are clear evidence that microscale SA offers unique advantages over current micro-assembly methods. Section 2.2 provides a background of SA models. SA models have focused in different aspects of a SA system; with the purpose of optimizing its performance. There is an extensive amount of literature on microscale SA. Therefore, the scope of this summary is limited to what is needed for understanding the motivation, and background behind the present work.

\subsection{Successful Implementations of Microscale SA}

The present summary of SA works covers component-to-substrate and component-tocomponent types of self-assembly. "Component-to-substrate" SA is where initially-separated components assemble onto the receptor sites on a substrate. On the other hand, components assemble to each other in "component-to-component" SA. Both SA types involve the same mechanisms. "Component-to-substrate" SA has a fixed frame of reference, which simplifies studying the process and quantifying interaction conditions.

The "origami" concept of self-assembly for microstructures with self-folding hinges is also referred to as SA (Bassik, Stern, \& Gracias, 2009; D. H. Gracias, Boncheva, Omoregie, \& Whitesides, 2002; Harsh \& Lee, 1998). However, this type of SA is of different nature than the 
component-to-component and component-to-substrate SA types. While all SA processes are based on energy minimization to acquire a desired final state, origami-SA involves dramatically reduced degrees of freedom. Generally, origami-hinges can only move in one direction; and it does not involve stochastic interactions between free-standing components. Therefore, this origami-SA lies outside of the scope of this dissertation and the present summary.

Microscale SA systems have most commonly implemented capillary forces as bonding mechanism (C.J. Morris et al., 2005; Srinivasan et al., 2001; W. Zheng \& Jacobs, 2005), and the present work will also utilize capillary SA. Other types of SA mechanisms involve magnetic (Fonstad, 2002; Shet, Mehta, Fiory, Ravindra, \& Lepselter, 2004), electrostatic (K.-F. Bohringer, Goldberg, Cohn, Howe, \& Pisano, 1998) and fluidic (Tolley, Baisch, \& Krishnan, 2008) forces. The following section covers mostly capillary SA systems, which comprise most of the relevant SA implementations overall. The parametric self-assembly model may also have application to processes using other assembly forces. However, a complete parametric model should account the impact-absorption capabilities that a capillary liquid bond has (and solid-solid interfaces do not, such as magnetic and electrostatic bonds). The analysis of differences between different bonding mechanisms will not be assessed in this work. The "Future Works" section of this manuscript describes on how the results from this work can be utilized towards addressing this topic.

\subsubsection{Component-to-Substrate SA}

Yeh and Smith first proposed the concept of fluidic self-assembly (FSA) (Yeh \& Smith, 1994), which led the first successful implementations of component-to-substrate SA at the microscale. In FSA, components are suspended in a fluid (aqueous) medium. Agitation creates stochastic interactions between components and assembly sites. Only a fraction of these 
interactions corresponded to parts arriving with correct conditions allowing for assembly. Bonding was achieved through hydrophobic interactions within an aqueous medium. By rendering binding surfaces hydrophobic, the medium liquid (water) "repels" such surfaces. Hence, binding surfaces that contact each other (from random interactions) stay bonded in order to achieve a lower energy state. An example of hydrophobic bonding is illustrated in Figure 3.

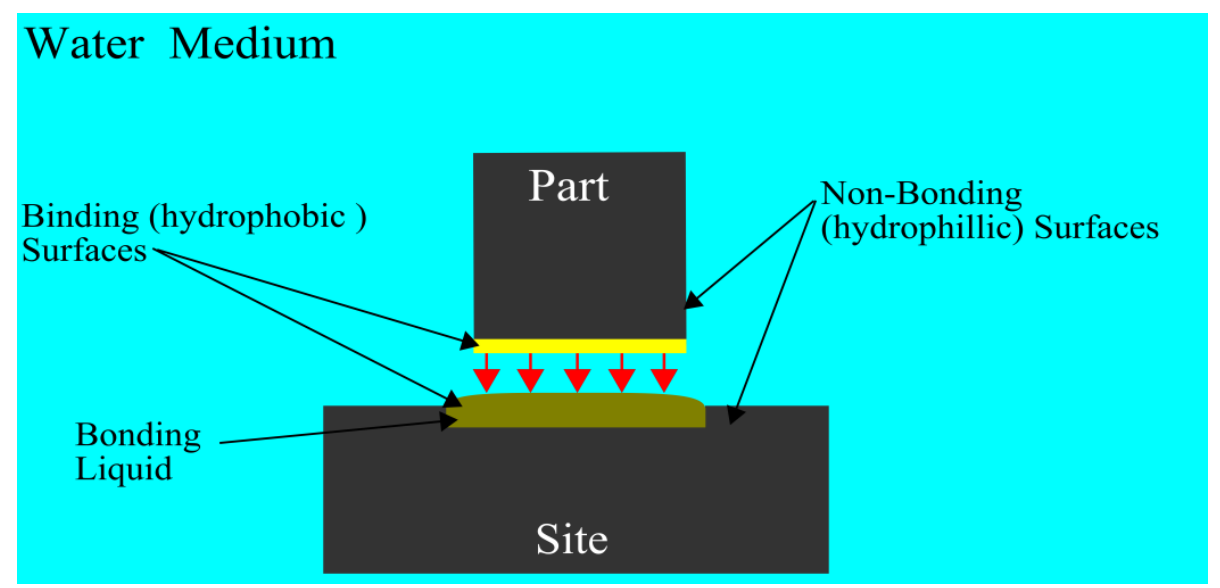

Figure 3 Hydrophobic bonding between a part and an assembly site. The binding surfaces are repelled by the water medium, so that when contacting each other, achieve a stable state. This condition is an optional configuration for capillary SA. Capillary bonds are also possible in air medium, and with different surface compositions.

In the early FSA works, many of the hydrophobic interactions were designed through liquid bonding agents (oils) that only remained stable while submerged under a liquid medium. Xiong et al demonstrated the use of a liquid polymer as a capillary bond agent, which would then be cross-linked by heat polymerization to create a permanent fixture (Xiaorong Xiong, Hanein, Wang, Schwartz, \& Bohringer, 2001). For many capillary SA works, a parallel process for easily coating many hydrophobic sites with oil, or solder has been implemented (Biebuyck \& Whitesides, 1994). Solder-based bonds allow electrical connections between substrate and assembled components; thus creating functional electronic assemblies (Jacobs, Tao, Schwartz, 
Gracias, \& Whitesides, 2002; Wei Zheng \& Jacobs, 2004) . Such solders are typically lowmelting point solders, which allow their implementation under aqueous solutions.

There were additional limitations of these early FSA systems. Some key issues were not addressed; but instead, the systems were simplified for preventing such issues from interfering. Firstly, assembly parts had 2D geometry, as they were much thinner than they were wide. Such allowed for a 50\% probability for parts to be correctly oriented upon arriving at the sites. Therefore, the assembly probability was enhanced. Moreover, parts were kept at very low kinetic energies, thus avoiding the disruptive effects when such has a magnitude comparable to the bonding energy.

Nevertheless, the early FSA works laid grounds for an extensive amount of following works that implemented microscale SA in different ways. For instance, successful microscale SA implementations have been demonstrated using other forces besides capillary, such as magnetic (Golosovsky, Saado, \& Davidov, 1999; O’Riordan, Delaney, \& Redmond, 2004), electrostatic (K.-F. Bohringer et al., 1998), fluidic (Tolley, Krishnan, Erickson, \& Lipson, 2008), and cellular forces (McNally, Pingle, \& Lee, 2003). Furthermore, while many microscale SA systems have utilized a liquid medium, others have been demonstrated in air. Bohringer et al proposed a "dry" microscale SA process; starting by laying components on the substrate with assembly sites. This approach simplified the transport step: which is to move the components towards the vicinity of sites. Then, ultrasonic agitation of the substrate forced components to stochastically align with the assembly sites, while bonding was achieved through electrostatic force fields (K.-F. Bohringer et al., 1998).

Given the stochastic nature of SA interactions, the alignment and bonding steps of any SA process are equipped with a strategy for selective bonding. That is, only "binding" surfaces 
should bond, while "non-binding" surfaces are not meant to bond even when they come into contact. For instance, Bohringer's “dry” SA system (K.-F. Bohringer et al., 1998) utilized the shape-matching concept to achieve selective bonding. This shape matching concept is a common approach to ensure that components bond only when appropriately oriented (J. Fang \& Bohringer, 2006; Ramadan, Uk, \& Vaidyanathan, 2007; Sharma, 2007; Smith, 2000; Stauth \& Parviz, 2006). Moreover, hydrophobic interactions (implemented by capillary-based SA systems such as FSA) are another common way of ensuring high bonding selectivity during stochastic interactions.

Despite all the efforts for enhancing selectivity, component-to-substrate SA requires a large number of interactions for achieving a desired amount of assemblies. Verma et al. proposed a component-recirculation system for creating more interactions without adding more components or fluid agitation (Verma, Hadley, Yeh, \& Smith, 1995). On the other hand, enhancing the probability of assembly for each individual interaction is another approach for raising process performance. Later SA works have proposed innovative solutions for such enhancements. Section 2.1.3 below reviews some noteworthy examples. Additionally, modeling efforts have also addressed solutions for enhancing assembly probability. These are reviewed in Section 2.2.

\subsubsection{Component-to-Component SA}

SA has been also implemented for assembling free-floating components to each other. Through this approach, components can be assembled into structures with more complex geometries. For instance, components can interact on more than one dimensional plane, thus creating 3D structures. Terfort et al. and Breen designed component-to-component processes for assembling millimeter and centimeter-scaled components, into 3D structures (Breen, 1999; 
Terfort et al., 1997). Similarly to FSA, these processes employed shape-matching to align components into 3D structures; and binding surfaces were rendered hydrophobic to bind through capillary (hydrophobic) interactions (Wei Zheng \& Jacobs, 2004).

SA interactions within a three-dimensional space are often challenged by the multiple degrees of freedom that reduce the probability of proper-alignment. Having motion constrained to a $2 \mathrm{D}$ plane increased inter-component interaction frequency, and reduces the number of possible orientations (thus improving the probability of assembly for each interaction). Bowden et al achieved this by floating components on a fluid-fluid interface for a component-tocomponent SA process (Bowden, Terfort, Carbeck, \& Whitesides, 1997). Component-tocomponent SA on fluid interfaces has been further demonstrated elsewhere (Grzybowski, Stone, \& Whitesides, 2000; Wu, Bowden, \& Whitesides, 1999).

\subsubsection{Enhancing Capabilities of Microscale SA and its Applications}

Later SA works that produced functional assemblies have illustrated the potential for SA to become a successful micro-assembly process. Many efforts have also succeeded at augmenting the strengths of microscale SA by adding more complex process capabilities. While this text offers a brief review on this topic, other reviews are useful for complementing the information given here (Cohn et al., 1998; R. Knuesel \& Park, 2012; M Mastrangeli, Abbasi, et al., 2009).

Jacobs et al. used capillary SA to assemble GaAs LED devices on a flexible substrate. Besides showing a functional product, this work demonstrated the integration of components and substrates that come from incompatible fabrication processes (J. Chung, Zheng, Hatch, \& Jacobs, 2006; Jacobs et al., 2002). Furthermore, the authors also demonstrated possible on-demand SA of multiple component batches into a common substrate. The ability to sequencing assembly of 
different part types has also been demonstrated through programmed electrochemical activation of bonding agent (X. Xiong et al., 2003); heat polymerization of oiled binding sites (J. Chung et al., 2006); and heat-activation of different melting-temperature solders (Liu, Lau, \& Yang, 2007).

Gracias et al succeeded at creating a 3D functional network of LED devices through component-to-component SA. Capillary forces provided inter-component bonding, as soldercovered contact pads came into contact under fluid agitation (D. H. D. Gracias, Tien, Breen, Hsu, \& Whitesides, 2000). The use of liquid solder as bonding agent and electrical contact has proven useful in several functional assemblies (Jacobs et al., 2002; Oliver, Clark, Bowden, \& Whitesides, 2001; Stauth \& Parviz, 2006; Wei Zheng \& Jacobs, 2004). Other microscale SA systems have demonstrated functional assemblies of different kinds, such as LED arrays (D. H. D. Gracias et al., 2000; X. Xiong et al., 2003; Yeh \& Smith, 1994; Wei Zheng \& Jacobs, 2004), piezoelectric actuators (J. Fang, Wang, \& Bohringer, 2006), thermo-electric coolers (Nathan B. Crane, Mishra, Murray, \& Nolas, 2009), solar cells (Robert J Knuesel \& Jacobs, 2011), live-cells (Zhang et al., 2005), and components on flexible substrates (Jacobs et al., 2002).

Lastly, interface-based SA efforts have also been developed into high-yield processes of functioning assemblies (RJ J Knuesel \& Jacobs, 2010; Robert J Knuesel \& Jacobs, 2011; Park, Xiong, Baskaran, \& Böhringer, 2011). As discussed previously, SA at the interface reduces the degrees of freedom of components, thus offering -with minimal effort- higher process rates and more precise alignments.

\subsection{Microscale SA Models: Motivation and Previous Work}

As discussed in Chapter 1, SA has been found difficult to implement at mass-production level. One approach for facilitating SA implementation is to understand further how to maximize 
SA performance. It is important to maximize SA process performance in order to make it a feasible (and competitive) micro-assembly process. Design tools, such as predictive models, would serve for this purpose, providing guidelines on how to maximize process performance.

The general goal when maximizing SA system performance is to maximize yield of successful assemblies. Given the stochastic nature of an SA process, this goal has been achieved by either maximizing the number of interactions over a given time, and/or by increasing the success rate (assembly probability) of each interaction. Such tasks have been targeted intuitively. For instance, Smith fabricated trapezoidal FSA components. Such geometry allowed for parts to orient themselves correctly as they travelled towards the sites with minimum drag force. Therefore, assembly probability was enhanced (Smith, 1994). Moreover, Fang and Bohringer introduced the DUO-PASS process, where the final bonding step was preceded by a prealignment step. Hydrophobic interactions and substrate agitation allowed for parts to spontaneously orient correctly, thus enhancing assembly probability.

General guidelines for maximum assembly probability cannot be provided through intuitive solutions. Instead, the use of predictive models enhances the understanding of specific effects. Moreover, methods for controlling process parameters do not scale well from laboratory demonstrations to mass- production. Hence, it is important to have appropriate parametric models of a general SA process. Such would guide the transition from a laboratory to a factoryscale process.

This summary demonstrates the different approaches for modeling different aspects of an SA process. SA models can be practically classified into those which focus on calculating the bonding strength and stiffness and those which focus on predicting the process rates and outcomes. 


\subsubsection{Bond Models}

SA bonds are designed for providing a minimum energy state to the desired position and alignment of a part in relation to the bonding site. They provide "pull-in" forces that correct a certain degree of rotational and translational misalignments. While based on static conditions, bond models focus on optimizing bond parameters for improving aspects such as alignment forces and bond energy. The most basic requirement of a self-assembly model is that the bonds are stronger than the disruptions. Commonly this is considered by designing bonds to be much larger than competing forces, such as gravitational forces. However, bond models do not consider the beneficial effects that competing forces have on SA performance. For example, agitation forces prevent unwanted bonds from forming, thus enhancing the yield of desired bonds. An analogy to chemistry is when temperature effects are not considered on a certain reaction. Nevertheless, bond models provide useful parametric relations for optimizing bond energy. The most commonly-studied parameters are bond-geometry and bonding surface composition.

Often, assembly bonds require unique component orientation because most components are not functionally symmetric. For the "shape-matching" approach, binding surfaces geometry is designed so that they can only come into contact at specific (or even unique) orientations. Figure 4 illustrates how different bond geometries offer one or more stable orientations (i.e. with minimum energy states).

Bohringer et al derived an energy model capable of investigating how different bonding site geometries affect the bonding energy; and furthermore, the magnitude of the alignment forces (K. F. Bohringer et al., 2001; Xiaorong Xiong et al., 2004). These models are $1^{\text {st }}$ order approximations of the surface energy involving bonding surfaces of a component and it's 
receptor site. Knowing that the total energy change is the sum of all surface energies involved, the models calculate how much energy reduction is offered by certain bonding pad geometry when the components are bonded relative to when they are separate.

(a)

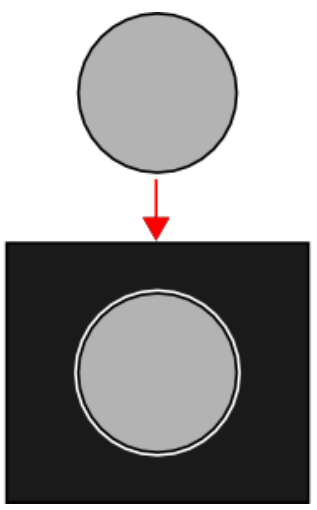

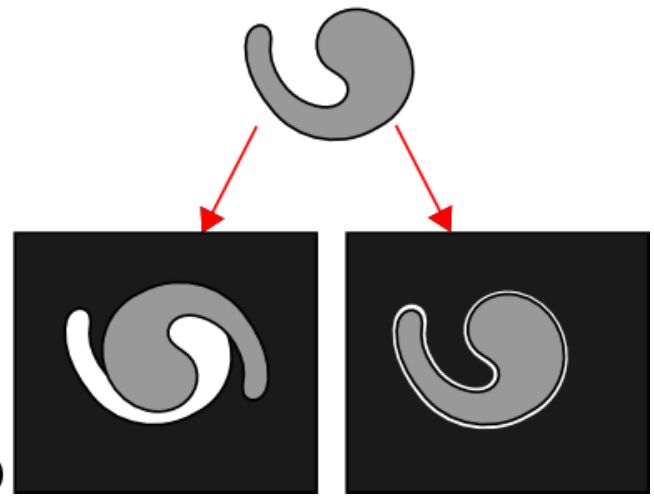

(b)

Figure 4 Effects of bond geometry on minimum energy states. Different bonding surface geometries can provide (a) one or (b) more stable configurations (K. F. Bohringer et al., 2001)

Similarly, Srinivasan et al modeled the effects of bonding site geometry using a finite element analysis program, which is a more computationally expensive approach. In this work, Srinivasan et al also experimented the effects that different bonding geometries have on assembly yield, and alignment precision (Srinivasan et al., 2001). Results from these works suggested that rectangular sites offered highest degrees of precision and assembly yield. However, rectangular sites offer 2 or possibly 4 different stable orientations.

The software used by Srinivasan et al, and many of the bond-modeling works is called Surface Evolver (Brakke, 1992). This program assumes quasi-static conditions, while generating numerical solutions for the lowest energy state of fluid interfaces. One can define boundary conditions such as solid constraints, liquid volumes and external force fields (e.g. gravity).

Several efforts have used Surface Evolver to illustrate how misalignment between a part and a binding site affects the magnitude of alignment forces (Greiner et al., 2002; Ramadoss \& Crane, 2008; Xiaorong Xiong et al., 2004). As a general conclusion from these efforts, alignment 
forces are proportional to the degree of misalignment (until the point where contact is lost). The model proposed by Lu et al looked at the dynamics of these alignment forces. The authors illustrated how the alignment forces change over time, while the part moves towards a minimumenergy state. (Lu et al., 2006). Lastly, Zhang et al measured effects of misalignment on alignment forces while considering a unique application: SA of live cells (Drosophila embryos) (Zhang et al., 2005).

Another important factor that defines bond energy is the surface composition of binding surfaces. The magnitude of binding energy offered by different surface compositions has been investigated by Onoe et al (Onoe et al., 2004). Here, the authors measured and calculated the magnitude of energy-minimization provided by different combinations of liquid and solid surfaces. The authors concluded that gold surfaces coated with an octadecanethiol self-assembled monolayer (SAM) offered the highest selectivity (i.e. lowest and highest energy states for desired and undesired contacts, respectively). Such calculations were consistent with experimental measurements of bonding yield.

Bond-models have provided significant lessons for optimizing bond energy. Higher bond energy allows for better alignment-precision, and bond selectivity. Hence, bond models play a role when binding surfaces are already in contact. On the other hand, process models play a role in optimizing component-interactions.

It is important to note that some key assembly parameters have not yet been addressed by these models. While bond models can be optimized for maximum bond energy, it is an issue when bond energy is much larger than kinetic energy. Low kinetic energy conditions compromise bonding selectivity. I.e., not only correct assemblies are allowed, but also incorrect ones. Such conditions are not ideal for achieving long-range order because all parts will stick. 
This is a particularly difficult issue when an assembly requires assembly selectivity between multiple bonding types. In nano-scale self-assembly, improved order is often achieved, when the bond energy is comparable to the thermal or agitation energy. As early as 1938, Langmuir recognized the positive effects on bonding selectivity from balancing bonding and agitation energies (Langmuir, 1938). Mermoud et al. modeled the same agitation/bonding energy balance in the context of microscale SA (Mermoud et al., 2009).

\subsubsection{Process Models}

These models focus on predicting process performance by, most commonly, calculating process rate and yield. The first microscale SA process model was a first-order chemical reaction model that considered all intermediate states from an unassembled precursor to a full assembly. It assigned assembly probabilities - based on component availability to calculate process rate (Hosokawa et al., 1994). The authors evaluated their models with subsequent experiments (Hosokawa, Shimoyama, \& Miura, 1996). These works of Hosokawa et al were the first to introduce the analogy of microscale SA to molecular kinetics.

Subsequently, Zheng and Jacobs proposed an approach to determine the rate parameter of a first-order chemical reaction model (W. Zheng \& Jacobs, 2005). Input parameters were the current number of unassembled precursor components. The authors obtained the process rate by regression of experimental data, and showed high correlation with the model equation. This was useful approach to study the effects of changing the relative quantities of assembly components. Similarly to this work is that of Napp et al (Napp, Burden, \& Klavins, 2006). Both models required prior-experimentation whenever any (process and/or component) parameter is changed. Additionally, these models did not capture the physics of inter-component interactions. Hence, they do not offer much information about how to increase the success rate of each interaction 
(they only offer information for how to increase the number of interactions). As a result, such models were not able to predict what would happen if, for instance, the agitation energy or the container volume is changed.

Mermoud et al proposed a multi-level modeling approach to predict assembly yield and capture the directionality of the assembly process (Mermoud et al., 2009). Three models simulated self-assembly at different levels. For all levels, the model inputs were agitation energy, maximum bond energy, and the tolerance for misalignment. Any collision between components formed an assembly at a certain magnitude of misalignment; and, the misalignment tolerance defined which assemblies broke-up.

1. First, a spatial agent model tracked the position orientation and velocity of every component. This model required extensive computation for tracking the collisions and breakups for a large number of components.

2. Secondly, a non-spatial model simulated the outcome of every interaction by just considering a geometric-based probability for components to collide, and randomly generating possible misalignment states. Without tracking the dynamics of every component, this non-spatial model implemented a Monte-Carlo approach to calculate which assemblies broke-up due to large misalignments. Both spatial and non-spatial models relied on a large number of iterations to acquire meaningful statistics.

3. Lastly, a macroscopic-level approach captured the state-space of all possible assemblies through a system of difference equations. This model simulated assemblies through a geometric-based assumption of the probability for single components to assemble into any of the possible (discretized) energy states 
(which range from perfectly aligned to the highest misalignment possible, defined by the misalignment tolerance value)

This modeling approach did not cover how the misalignment tolerance related to component configuration (geometry) and bonding mechanism (e.g. liquid capillary bonds tolerate a certain degree of misalignment, based on surface tension and volume properties). Instead, the authors assumed an arbitrary value, and also evaluated model outputs for a range of possible values.

Another modeling effort implemented an agent based model following the same approach as \#1 (above) proposed by Mermoud et al. However this new effort simulated interactions in three dimensions, and considered component geometry and physics of motion and collision (Massimo Mastrangeli, Van Hoof, Baskaran, Celis, \& Bohringer, 2010). Such model is a powerful tool for optimizing process rate and yield by varying component quantities and workspace volume. However, this model also relied on previous knowledge of the misalignment tolerance property. Both modeling efforts (Massimo Mastrangeli et al., 2010; Mermoud et al., 2009) offer the most relevance to the present study, which focuses on relating the misalignment tolerance value to controllable parameters, such as component geometry, and bonding mechanism. However, they do not readily provide basic scaling rules to guide part and process design.

\subsection{Current Contribution to SA Process Models}

Prediction of SA process rate is essential for simplifying the implementation process of microscale SA. As stated previously, process rate can be increased by either increasing the rate of stochastic interactions, or the probability that each interaction successfully assembles (i.e. probability of assembly). The rate of stochastic interactions is limited by the hypothesized 
requirement that the kinetic energy remain below the bonding energy in order for successful bonds. This means that the primary means of controlling the self-assembly rates is by increasing the assembly probability. Prior experimental works have used both approaches, but most processes are currently developed using only bonding models to verify the stability of the bonds. The incorporation of process models into the process development would guide selection of assembly conditions (agitation type and intensity) to achieve better assembly rates and accuracy.

The prior works reviewed above have proven powerful for understanding the effects of process-level parameters on SA yield, rate, and even directionality. Such process level parameters include agitation energy, theoretical bond energy, workspace volume, number and characteristic size of components. However, the lack of detail about the physics of interactions hindered the models' ability to evaluate component-level parameters. These parameters include specific geometry of components (size, orientation and number of bonding and non-bonding surfaces), surface tension and alignment properties of bonding mechanism. Instead, some of the afore-mentioned process models compiled the effects of such parameters onto one scalar, as a measure of the components' ability to assemble, and remain assembled (Massimo Mastrangeli et al., 2010; Mermoud et al., 2009). Moreover, the rest of process models only predicted assemblies after being calibrated through experimentation (Napp et al., 2006; W. Zheng \& Jacobs, 2005).

The present work focuses on deriving basic relations between component-level parameters and SA process performance in simple parametric models that provide critical understanding to the process designer in developing a fast, accurate, and reliable assembly system. The present experimental study provides understanding of the parameters that were overly simplified (or were not evaluated) in previous modeling efforts. Furthermore, the results from this work are meant to complement existing process models, which covered the effects of 
process-level parameter (Massimo Mastrangeli et al., 2010; Mermoud et al., 2009). Accordingly, combining evaluated effects of process-level parameters with those of component-level parameters (evaluated here) will provide a complete SA system design tool for predicting outcome of inter-component interaction. 


\section{CHAPTER 3:}

\section{EXPERIMENTAL PROCEDURES}

As discussed in Chapter 1, the present work focuses on characterizing a basic SA interaction. A basic SA interaction is defined as the event where one part lands on one site (see Figure 2). All assemblies arise from these basic interactions and so they are the unit cell of an SA process. More complex interactions can exist in an actual SA process. For instance, two parts could simultaneously land on one site; or a part could land on a partially occupied site. These types of interactions are not characterized by the present work. However, the resultant characterization of the simple interactions serves as groundwork for a more complex study that covers all possible interactions.

The present work proposes a parametric model of basic part-site interactions. Chapter 4 introduces the model in detail, how it was derived and its assumptions. This chapter focuses on the experimental methods for evaluating such model. The outcome of basic SA interactions will be defined (in Chapter 4) as a function of three key parameters that were considered as influential to the outcome of basic SA processes. These parameters are: ratio of kinetic energy $E_{k}$ over binding energy $E_{b}\left(E_{k} / E_{b}\right)$, component orientation $\rho_{o}$, and angle of incidence $\varphi$. This parameterization scheme is based on some important simplifications that would have to be accounted for in order for the parametric model (introduced in Chapter 4) to completely become a general model of SA interactions. First, the parameterization scheme considers only the landing location of the center of the part. Actual parts could potentially assemble with the center outside the binding site. Hence, a model based on these parameters alone would not completely predict 
the effects of a more realistic interaction (which has larger misalignment). Chapter 4 introduces a scalar factor that accounts for such offset in the model. Secondly, the energy parameter $\left(E_{k} / E_{b}\right)$ allows the model to consider any microscale SA process, regardless of the bond mechanism (capillary, magnetic, electrostatic, etc). However, the capillary bonds are often comprised of liquid-solid interfaces. Liquid bonds offer impact absorption, thus allowing for a greater tolerance to high kinetic energies. Moreover, the viscoelastic properties of the liquid bond will also play a role in the bonding process, especially at high $E_{k} / E_{b}$ values by dissipating some of the energy. Therefore, a complete SA interactions model shall complement the present parameterization scheme with a method for accounting for impact-absorption effects.

The present chapter explains the procedures for controlling each one of the aforementioned parameters $\left(E_{k} / E_{b}, \rho_{o}\right.$ and $\left.\varphi\right)$, and for running experiments. Chapter 4 discloses results and analysis of the measured effects that $\varphi$ and $\rho_{o}$ have on the outcome of the basic partsite interactions. Chapter 5 presents an optimized experimental procedure for evaluating the effects of energy ratio $E_{k} / E_{b}$. Chapter 4 tests were performed in a water medium, while the energy-ratio tests were performed in air medium.

The experimental system allowed basic SA interactions while controlling key conditions (experimental variables). This is accomplished by dropping the parts one-by-one, on top of an array of assembly sites. Parts had equal probability of landing on any site. The geometry of parts controlled the orientation probability $\rho_{o}$. The ambient viscosity and the height-of-dropping controlled the kinetic energy $E_{k}$. The surface area and material compositions controlled the bonding energy $E_{b}$. Lastly, assembly sites were rotated with a certain angle of incidence $\varphi$ with respect to the vertical motion of falling parts. Controlling the angle $\varphi$ allowed for evaluating whether the outcome of SA interactions is path independent. 
Experiments that studied $\varphi$ and $\rho_{o}$ effects were performed under a low $\mathrm{E}_{\mathrm{k}} / \mathrm{E}_{\mathrm{b}}$ condition $(<0.1)$. This condition allowed for reducing energy effects significantly. Hence, assembly sites were mounted inside a water-filled container. The water medium caused parts to fall at low and constant (terminal) velocity. On the other hand, experiments for studying energy effects were performed in a dry (atmospheric air) environment. This allowed for adjusting velocity (hence, $E_{k} / E_{b}$ ) within a broad range of values from just above 0 , to beyond 1 .

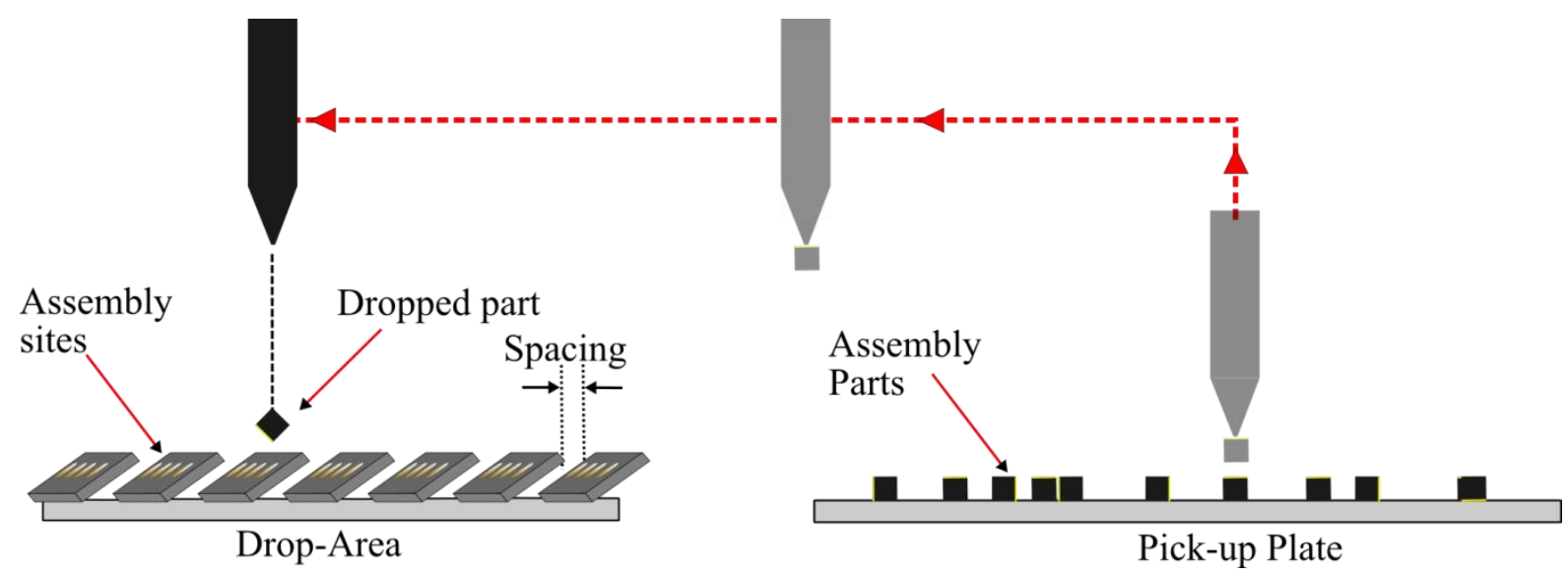

Figure 5 Schematic of experimental SA system

Figure 5 illustrates a schematic of the experimental system components. A CNC system was programmed to control a gripper which picked up, and dropped parts. One-by-one, parts were picked-up from the pickup-plate, and dropped over the area of substrates with assembly sites. The dropping locations were selected to approximate a uniform distribution of parts over the assembly site areas. The goal of the assembly site substrates and part-dropping system design was to allow for every site to have equal probability of a part landing on it. The assembly site arrangement allowed for non-assembling parts (and parts that did not land on any site) to fall through and avoid interfering with subsequently dropped parts. Additionally, any non-binding surface area directly above the site is undesired. Parts landing on such area above a site could bounce off, or slide onto an assembly site. Such type of interaction (considered a "secondary" 
interaction) could cause an unexpected assembly. Chapter 4 provides a more detailed discussion how the space above assembly sites was varied to evaluate the effects of secondary interactions.

Each SA test involved a sequence of individual interactions. The fraction of interactions that turned into assemblies was measured by observing assembly sites regularly. Similarly, observations also considered the fraction of parts assembling incorrectly (parts bonding with a non-binding surface). Fraction of correct assemblies is a measure of assembly probability, $\rho_{a}$. Chapter 4 will explain how $\rho_{a}$ is directly related to initial assembly rate of a basic SA process. Additionally Chapter 4 gives further detail about the proposed parameterization of an SA interaction, and the experimental framework for evaluating such parameters.

The present chapter explains many of the procedures implemented for running SA tests (as described above). The following two sections describe the methods for controlling binding energy, $E_{b}$ and kinetic energy, $E_{k}$. Next, section 3.3 depicts the fabrication process for test samples (assembly parts and sites). Additionally, the Pick-and-Drop system, and gripper designs are described in section 3.4.

\subsection{Binding Energy Control}

Each dropped part has certain probability of landing on a site. It also has certain probability of landing with its binding surface facing the site. Consequently, each part can experience one of several possible contacts. Figure 6 displays all possible contacts that can occur during a part-site interaction. The contacts between binding surfaces are labeled as "assembly contacts" (Figure 6-a). A negative energy change for a specific contact type signifies a more stable state than when the component was not in contact (. Furthermore, the energy change provided by the binding energy must also have larger magnitude than the kinetic energy. Otherwise a component is not expected to find stability upon contact. On the other hand, it is 
possible for the binding energy of undesired contact types (see Figure 6 b-c) to be negative (and potentially with larger magnitude than the kinetic energy). In other words, the energy state of parts bonded to other surfaces may still be more stable than no contact at all (i.e. fully surrounded by the medium). For this reason, an SA process is designed to provide a large contrast of binding energy between the desired bonding configuration (i.e. assembly contact) and every other contact type. A large contrast provides a higher selectivity for desired assemblies, thus reducing potential defects.

Parts can also land partially over an assembly site. Hence, the design of the bond mechanism shall provide features to create a desired contact type over other types. For instance, in capillary SA sites are filled with a bonding liquid. A liquid binding site is a deformable bonding surface that can respond and stretch out when an edge or a corner makes contact (which is more likely than a component landing flat on a surface). Hence, a bonding fluid extends the range of interaction that generates assembly forces, thus further reducing the likelihood for a part to contact only a nearby non-binding surface. Additionally, non-assembly contacts (Figure 6-c, d) involve interactions with only solid surfaces. The collision energy between solid surfaces is not absorbed as much as with liquid surfaces; the contact area is typically very small, and the contact time is very brief. Consequently, undesired contacts are more sensitive to kinetic energy, which often causes parts to "bounce off" the solid, non-bonding surface.

$E_{b}$ is directly proportional to the amount of contact area. For the case of a part landing partially over a site, the assembly-contact $E_{b}$ is reduced by the fraction of contact area between binding surfaces. Inherently, there is a certain misalignment threshold above which $E_{b}$ is no longer enough to produce an assembly. This threshold may also depend on other parameters such 
as the kinetic energy, fluid volume, and arrival angle. Such dependence is further discussed in Chapter 4.

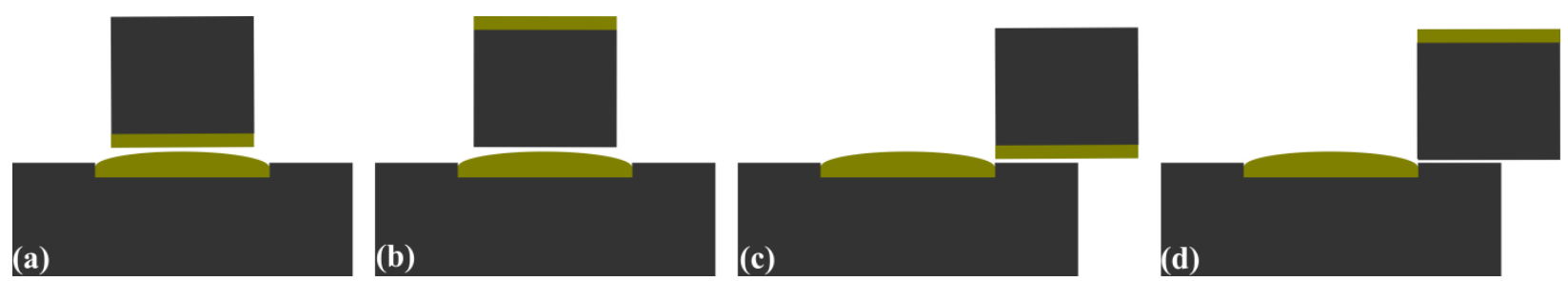

Figure 6 Types of part-site contacts. (a) Contact between binding surfaces, also labeled as an "assembly contact". (b) Contact between a site and the non-binding surface of a part. This is labeled as incorrect assembly contact. (c) and (d) are contacts when a part lands outside of a site.

The capillary binding energy $E_{b}$ can be approximated in terms of the individual interfacial tension components. If interacting surfaces are assumed to have a flat and smooth surface area $\left(a_{b}\right)$, then $E_{b}$ can be approximated by the difference between initial and final interfacial energies. Hence,

$$
E_{b}=\left(\gamma_{p s}-\gamma_{s m}-\gamma_{p m}\right) \cdot a_{b}
$$

Equation 1

The $\gamma$ terms are interfacial tension components. The subscripts $s, m$ and $p$ correspond to surface of the assembly site substrate, assembly medium and part surface, respectively. If surface curvature is significant, then a numerical model such as with Surface Evolver model may be required to improve the accuracy of the bond energy estimate.

The interfacial tension components $\gamma_{s m}$ (i.e. interface between bonding liquid and medium fluid) were measured with a Du-Noüy ring tensiometer (Sigma 701 from KSV Instruments Ltd, Finland). Subsequently, the difference $\left(\gamma_{p s}-\gamma_{p m}\right)$ was acquired by Young's Equation:

$$
\gamma_{s m} \cdot \cos (\theta)=-\left(\gamma_{p s}-\gamma_{p m}\right)
$$

Equation 2

where the angle $\theta$ was obtained through contact angle goniometry. Table 1 summarizes results of all contact angle measurements, and $E_{b}$ values. Advancing angles are formed as the contact line (i.e. interface between $s, m$, and $p$ surfaces) moved forward, and correspond to the energies 
required to form a bond (for the corresponding contact type). On the other hand, receding angles formed while the contact line moved away from the medium, and corresponds to the energy required for breaking a bond. The difference in $E_{b}$ energy between is a direct measure of the selectivity of a correct assembly over an incorrect assembly.

Table 1 Contact angle measurements for determination of binding energy $\left(E_{b}\right)$ for possible contact types.

\begin{tabular}{|c|c|c|c|c|c|}
\hline $\begin{array}{c}\text { Contact } \\
\text { type }\end{array}$ & Surfaces & $\theta$ & $\begin{array}{l}\gamma_{p s-}-\gamma_{p m} \\
\left(\mathbf{m J} / \mathbf{m}^{2}\right)\end{array}$ & $\begin{array}{c}E_{b} / a_{b} \\
\left(\mathbf{m J} / \mathbf{m}^{2}\right)\end{array}$ & $\begin{array}{c}E_{b} \\
(\mathbf{n J})\end{array}$ \\
\hline \multicolumn{6}{|c|}{ Water Medium Tests } \\
\hline \multirow{2}{*}{ Assembly } & \multirow{2}{*}{$\begin{array}{c}\text { p: SAM (thiol) } \\
\text { s: hexadecane } \\
\text { m: water }\end{array}$} & Advancing: $42.60^{\circ}$ & -37.40 & -88.20 & -22.1 \\
\hline & & Receding: $2.00^{\circ}$ & -50.77 & -101.58 & -25.4 \\
\hline \multirow{2}{*}{$\begin{array}{l}\text { Incorrect } \\
\text { Assembly }\end{array}$} & \multirow{2}{*}{$\begin{array}{c}\text { p: } \mathrm{SiO}_{2} \\
\text { s: hexadecane } \\
\text { m: water }\end{array}$} & Advancing: $137.05^{\circ}$ & 37.19 & -13.62 & -3.4 \\
\hline & & Receding: $85.46^{\circ}$ & -4.02 & -54.82 & -13.7 \\
\hline \multicolumn{6}{|c|}{ Air Medium Tests* } \\
\hline \multirow{2}{*}{ Assembly } & \multirow{2}{*}{$\begin{array}{c}\text { p: } \mathrm{SiO}_{2} \\
\text { s: water } \\
\text { m: air }\end{array}$} & Advancing: $21.82^{\circ}$ & -66.65 & -117.46 & -29.4 \\
\hline & & Receding: $2.00^{\circ}$ & -71.76 & -122.56 & -30.6 \\
\hline
\end{tabular}

* For air-medium tests, all part surfaces were binding surfaces. Hence, incorrect-assembly contacts were not possible. The subscripts $p, s$ and $m$ correspond to the part, site and medium surfaces, respectively.

\subsection{Kinetic Energy Measurements}

The water-medium parts reach terminal velocity before landing on a site. Digital videos with fixed framerate allowed for measuring terminal velocity. On the other hand, air-medium parts do not reach terminal velocity. Therefore, the height from which the part was dropped controlled the landing velocity. Digital imaging also provided velocity measurements for different drop heights. Figure 7 illustrates the measurement system, and sample images taken for measuring part velocity. The measuring system involved a CMOS digital camera (IDS UI2210SE) with a Computar M1214-MP2 lens. 


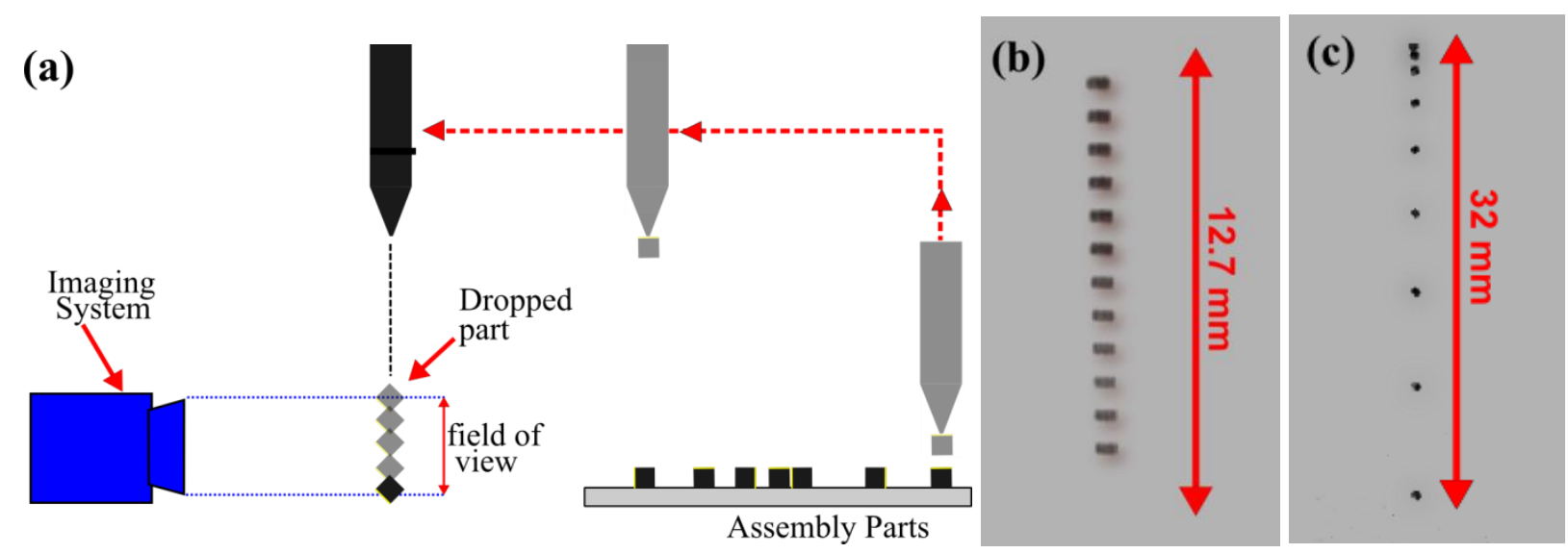

Figure 7 System for measuring part-velocity. Frames were extracted from videos that captured a moving part. Frames were overlaid, using Photoshop software to depict multiple frames of a part falling in (b) water, and (c) air media.

The water-medium parts were imaged at 75 frames per second with $224 \times 436$ pixel resolution (low resolutions allowed for increasing frame rate to desired value). For each video, velocity was calculated as the average distance between part intervals, divided by the time-period of one frame. Standard deviations of distance between part-intervals below 5\% confirmed that parts traveled at constant (terminal velocity). On the other hand, the air-medium parts were imaged at 91 frames per second with 136 x 375 pixel resolution. Parts are imaged right after released by the gripper, and while still accelerating (before reaching terminal velocity). Velocities were acquired by first measuring the distance traveled by the part at each time interval. Then the slope of the distance over-time plot provided a velocity value. Average velocities were acquired for each time interval from 10 repetitions (videos). Moreover, each time interval corresponded to a certain distance traveled (depicted in Table 2). Next, the kinetic energy $E_{k}$ was calculated by $E_{k}=0.5 \cdot m \cdot V^{2}$ where $m$ is the mass of the part, and $V$ is the measured velocity. Table 2 depicts resultant kinetic energy values for each test scenario.

Rotational energy of incident parts could affect the impact dynamics of part-site interactions, thus compromising their predictability. However, part-drop videos showed 
negligible rotational motion. (as image resolution allowed to observe). Additionally, horizontal motion was also negligible for each part-drop.

Table 2 Kinetic energy measurements.

\begin{tabular}{|c|c|c|c|}
\hline \multicolumn{4}{|c|}{ Water Medium Tests } \\
\hline Part Geometry & $\begin{array}{l}\text { Terminal Velocity } \\
(\mathrm{mm} / \mathrm{s})\end{array}$ & Kinetic Energy $(\mathrm{nJ})$ & $\mathrm{E}_{\mathrm{k}} / \mathrm{E}_{\mathrm{b}} *$ \\
\hline $500 \times 500 \times 500 \mu \mathrm{m}^{3}$ & $81.88 \pm 3.13$ & $0.976 \pm 0.001$ & 0.044 \\
\hline $500 \times 500 \times 400 \mu \mathrm{m}^{3}$ & $73.58 \pm 1.20$ & $0.630 \pm 0.00001$ & 0.029 \\
\hline \multicolumn{4}{|c|}{ Air Medium Tests } \\
\hline Drop Height (mm) & Velocity $( \pm 30 \mathrm{~mm} / \mathrm{s})$ & Kinetic Energy $( \pm 0.03 \mathrm{~nJ})$ & $\mathrm{E}_{\mathrm{k}} / \mathrm{E}_{\mathrm{b}} * *$ \\
\hline 0.91 & 62.16 & 0.562 & 0.02 \\
\hline 2.88 & 151.25 & 3.328 & 0.11 \\
\hline 6.06 & 264.18 & 10.154 & 0.33 \\
\hline 10.36 & 364.63 & 19.345 & 0.63 \\
\hline 15.80 & 471.32 & 32.322 & 1.06 \\
\hline 22.36 & 572.30 & 47.655 & 1.56 \\
\hline 30.04 & 677.13 & 66.712 & 2.18 \\
\hline
\end{tabular}

$* \mathrm{E}_{\mathrm{b}}=22.1 \mathrm{~nJ}$ corresponds to the formation energy of a correct assembly in water medium, as tabulated in Table 1.

$* * \mathrm{E}_{\mathrm{b}}=29.4 \mathrm{~nJ}$ corresponds to the formation energy of a correct assembly in air medium, as tabulated in Table 1.

\subsection{Preparation of Test Samples}

The samples used for SA tests were not functional devices. However, they were fabricated though standard micro-fabrication (silicon-based) processes. Such processes produced samples with similar size and surfaces, as actual devices would likely have. The scope of microscale SA covers sample sizes well below $100 \mu \mathrm{m}$. However, this work implements samples with dimensions of $500 \mu \mathrm{m}$, approximately. Such sizes were allowed by the available microfabrication equipment. These sample sizes were easily manipulated by the pick-and-drop system. Smaller sizes would have been unfeasible. Nevertheless, these sample sizes also fall within the microscale SA regime, and allow for achieving the goals of this present work. 
Assembly sites and parts were fabricated from $500 \mu \mathrm{m}$ silicon wafers (with mirrorpolished surfaces on both sides). The finished substrates were diced into strips. Each strip had a single array of sites (see Figure 5). Electrical properties were not relevant for this project. However, wafers used for these samples were rated for 1-10 $\Omega . \mathrm{cm}$. All sample fabrication processes were performed at the USF Nanotechnology Research and Education Center (NREC).

Following the fabrication of silicon strips, filling the assembly sites with bonding liquid provided the desired bonding energy, and self-alignment force (Greiner et al., 2002; Ramadoss \& Crane, 2008). The fabrication procedures depended on the medium where tests were performed. Accordingly, samples for Chapter 4 and Chapter 5 tests are labeled "Water-medium samples" and "Air medium samples", respectively. The following sections explain the fabrication procedure for each type of samples.

\subsubsection{Water-Medium Samples}

Water medium samples were designed for parts to travel at a low velocity. Bonding energy was achieved by rendering binding surfaces hydrophobic. As it was described in section 3.1, hexadecane oil was the bonding liquid for water medium assembly sites.

\subsubsection{Assembly Sites}

Site geometry is depicted in Figure 8, and fabrication steps are depicted in Figure 9. Photolithography was performed using $1.2 \mu \mathrm{m}$ thick positive photoresist (AZ 1812), applied by spin-coating technique. UV Exposure was performed with a Karl Seuss MA-56 mask aligner. The mask used to pattern the photoresist into assembly sites is illustrated in Figure A.1. A stepby-step summary of such procedure is listed below:

1. Spin coat photoresist (1,800 rpm for 40 seconds).

2. Soft-bake on hot plate at $90^{\circ} \mathrm{C}$ for 30 seconds. 
3. Expose G-line UV light at $20 \mathrm{~mW} / \mathrm{cm} 2$ for 4 seconds.

4. Develop in photoresist developer (AZ-300MIF) for 2 minutes.

5. Hard-bake on hot plate at $90^{\circ} \mathrm{C}$ for 30 seconds.

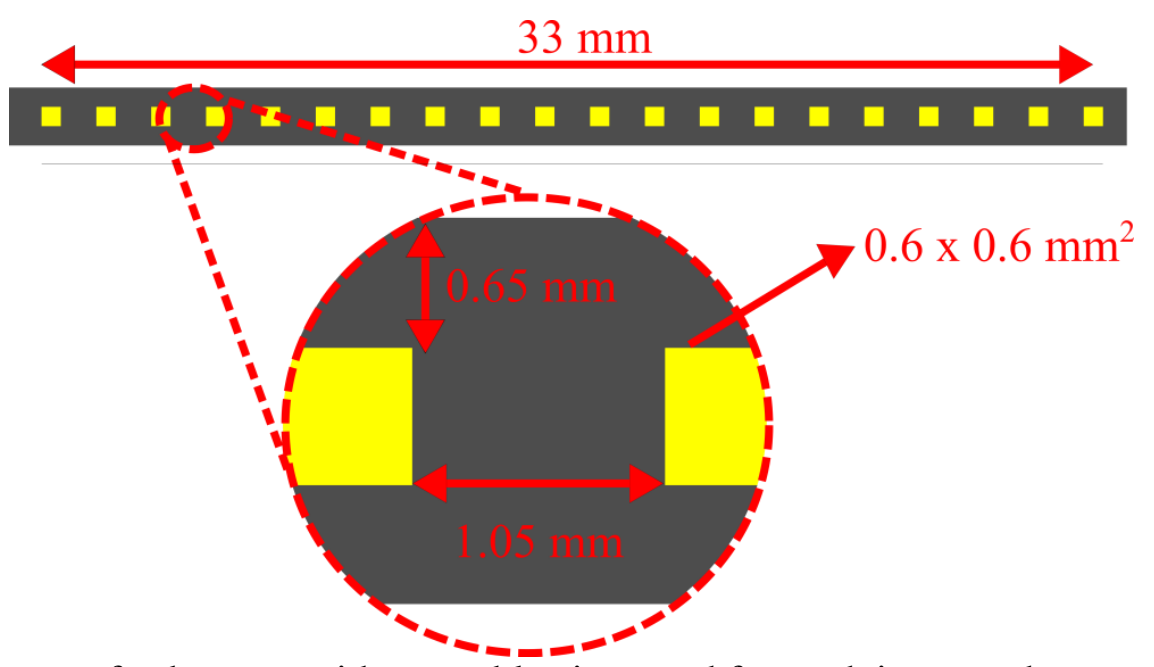

Figure 8 Geometry of substrates with assembly sites used for studying $\varphi$ and $\rho_{o}$ parameters. Image is drawn to scale.

After photolithography, recesses in assembly sites were created by etching $20 \mu \mathrm{m}$ of silicon with an Adixen AMS 100 deep reactive ion etching (DRIE) system. The process lasted 90 seconds. The etching recipe labeled "Si-HAR" (for high aspect ratio), and was supplied by NREC staff. It is important to note that the photoresist used (AZ-1512) was not designed for plasma etching. However, the low etching-time required for $20 \mu \mathrm{m}$-etch did not compromise photoresist functionality.

Subsequently, a gold (Au) film (200 nm thick) was deposited on the wafer using an Electron-beam evaporation system. Evaporation was performed at $0.1 \mathrm{~nm} / \mathrm{s}$ rate. Moreover, a 20 nm Chromium (Cr) layer was deposited before the Au layer, to ensure adhesion between Si and Au materials. Next, the photoresist was removed through a lift-off technique, consisting of a 12 hour acetone dip. Wafers were diced into strips using a micrometer-scale dicing saw. Each strip contained a linear array between 24 and 30 assembly sites. 

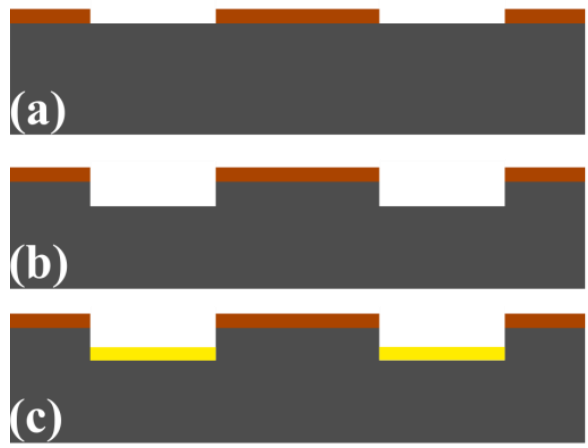

Silicon Wafer

Photoresist

Gold
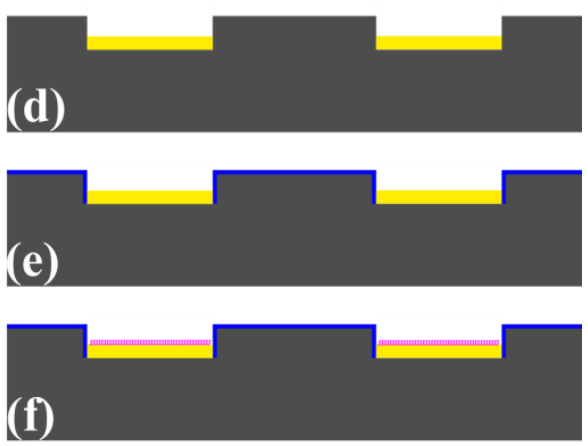

$\mathrm{H}_{2} \mathrm{O}_{2}$ (hydrophilic) 3535 SAM (hydrophobic)

Figure 9 Steps for fabricating water medium assembly sites. (a) Lithography (b) Deep reactive ion etching (DRIE) (c) E-beam physical vapor deposition (d) Photoresist removal (e, f) Surface treatments.

\subsubsection{Assembly Parts}

In summary, parts had both width dimensions equal to $500 \mu \mathrm{m}$, and height dimension corresponding to the wafer thickness. The part-orientation study (reported in Chapter 4) involved varying the height dimension, and the number of binding surfaces. Therefore, Chapter 4 describes in detail the range of part heights that were fabricated.

Similarly to sites, assembly parts were fabricated from the same type of silicon wafers. The blank wafers were coated with a $200 \mathrm{~nm}$ thick Au layer (and with an underlying $20 \mathrm{~nm}$ thick Cr layer), using the same e-beam evaporation procedure as for assembly sites. For parts having 2 binding surfaces, both sides of the wafer were coated with Au layer, (by running the deposition process once, for each side). Next, the wafer was diced using a micrometer-scale dicing saw.

\subsubsection{Surface Preparations}

After dicing strips with assembly sites, and assembly parts, both groups of samples were treated for surface rendering. Sites were treated in a Pyrex container; while parts were treated in a glass vial. Both parts and sites were dipped in $30 \% \mathrm{H}_{2} \mathrm{O}_{2}$ for 1 hour. This treatment grew a 
$\mathrm{SiO}_{2}$ layer on all exposed $\mathrm{Si}$ surfaces. Hence, this treatment rendered all non-binding surfaces hydrophilic. Next, samples were dipped in a $1 \mathrm{mM}$ solution of octadecanethiol in ethanol, for 24 hours. Such treatment formed a self-assembled monolayer (SAM) on the Au surfaces, thus rendering all binding surfaces hydrophobic. More details about the selective-coating process using self-assembled monolayer can be found elsewhere (Biebuyck \& Whitesides, 1994; Onoe et al., 2004; Sigma Aldrich, 2006).

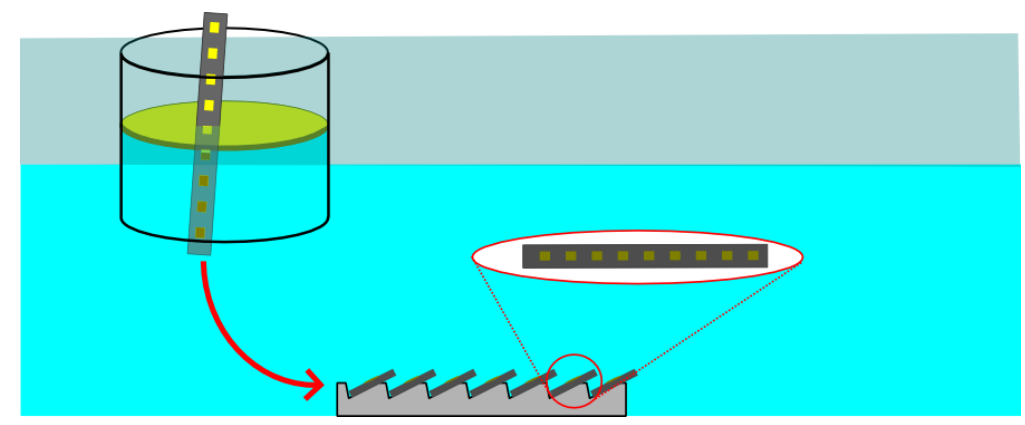

Figure 10 Illustration of pass-through interface procedure for selective oil-coating of assembly sites. The strip with hydrophobic assembly sites was passed through a confined oil layer, into the water medium. The oil selectively coated the sites, while repelled by the hydrophilic, nonbinding surfaces. This coating became the bonding liquid for SA tests under water medium.

After applying the above-described treatments, all non-binding and binding surfaces were rendered hydrophilic and hydrophobic, respectively. At this point, assembly sites were ready for bonding-liquid deposition. The strips with assembly sites were passed through a hexadecane oil layer, into the water medium. This pass-through-interface technique deposits the bonding liquid (hexadecane oil) on each assembly site, without covering the hydrophilic $\mathrm{SiO}_{2}$ of non-binding surfaces (M Mastrangeli, Ruythooren, Van Hoof, \& Celis, 2009; Srinivasan et al., 2001). The oil layer contained $24 \mathrm{ml}$ of hexadecane, and was constrained within a 4 in circular wall, as it is shown in Figure 10. Each assembly site strip was oriented horizontally while passing through the oil (the length of the strip was parallel to the interface). Lastly, after coating the assembly sites with hexadecane oil, the sites were mounted on the SA test mount. Strip orientation, oil volume, 
and constraint method were observed to be important in maintaining a consistent oil deposition pattern on all binding sites.

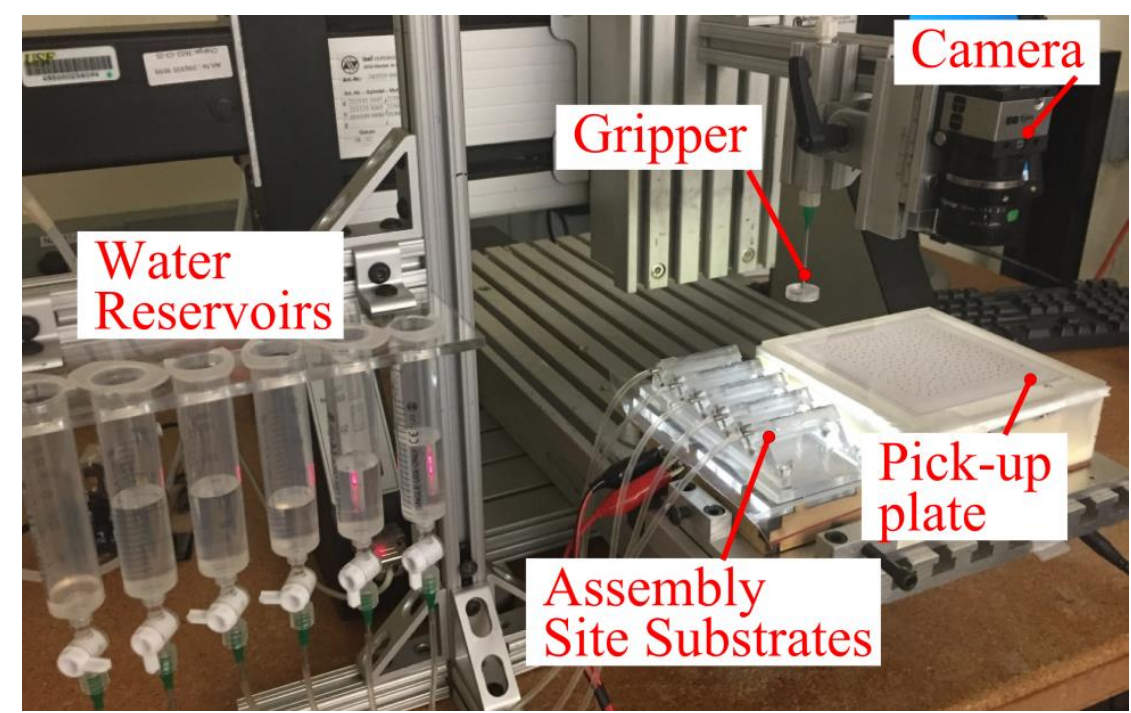

Figure 11 Experimental system for air-medium tests.

\subsubsection{Air-Medium Samples}

Air medium samples allowed for parts to travel at higher velocities. Contrary to watermedium samples, the binding surfaces of air-medium samples were designed as hydrophilic. The non-binding surfaces were designed as hydrophobic. Accordingly, the bonding liquid was water for this case. The assembly sites were fabricated as through via in the silicon substrate. This allowed for feeding water from the underside by means of hydrostatic pressure (see Figure 12). The relative height of the water reservoir defined the height of the bonding liquid (also called "site height", $h$ ). Each strip installed was connected to an independent water reservoir. A picture of the experimental set-up is shown in Figure 11.

\subsubsection{Assembly Sites}

The assembly sites for air-medium tests had the same surface area $(600 \mu \mathrm{m}$ x $600 \mu \mathrm{m})$, and a slightly smaller inter-site spacing as water-medium sites (see Figure 13). The revision in 
inter-site spacing was made to closely pack more sites in a single strip. The fabrication steps are depicted in Figure 12. All processes utilized the same equipment used for the water-medium samples. Photolithography was performed using $12 \mu \mathrm{m}$ thick positive photoresist (AZ 12xT). This photoresist was more adequate than the AZ 1512 for a longed silicon etching process. The mask used to pattern the photoresist into assembly sites is illustrated in Figure A.2.

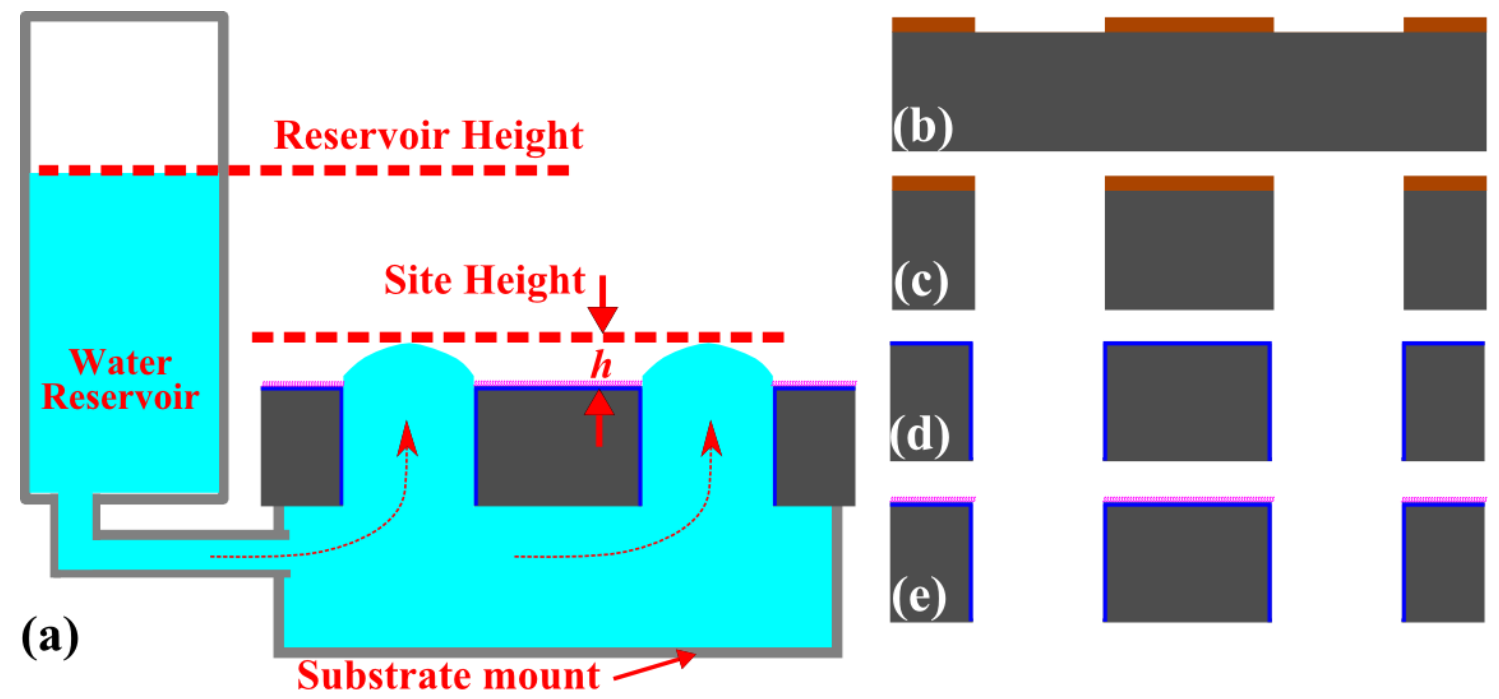

Silicon Wafer $\mathrm{H}_{2} \mathrm{O}_{2}$ (hydrophilic)

Photoresist s35s SAM (hydrophobic)

Figure 12 Procedure for fabricating air-medium assembly sites. (a) Method for filling assembly sites with bonding liquid (water). Hydrostatic pressure was created by relative height $h$ of reservoir level. (b-e) Fabrication steps of air-medium samples: lithography (a), deep reactive ion etching (DRIE) (c), hydrophilic treatment (d), Spin-coating (e).

The procedure for photo-resist application is listed below:

1. Spin coat photoresist (2,000 rpm for 40 seconds).

2. Soft-bake on hot plate at $115^{\circ} \mathrm{C}$ for 1 minute.

3. Expose G-line UV light at $20 \mathrm{~mW} / \mathrm{cm} 2$ for 8 seconds.

4. Post-exposure bake on hot plate at $90{ }^{\circ} \mathrm{C}$ for 2 minutes

5. Develop in photoresist developer (AZ-300MIF) for 2 minutes.

6. Hard-bake on hot plate at $90^{\circ} \mathrm{C}$ for 5 minutes. 

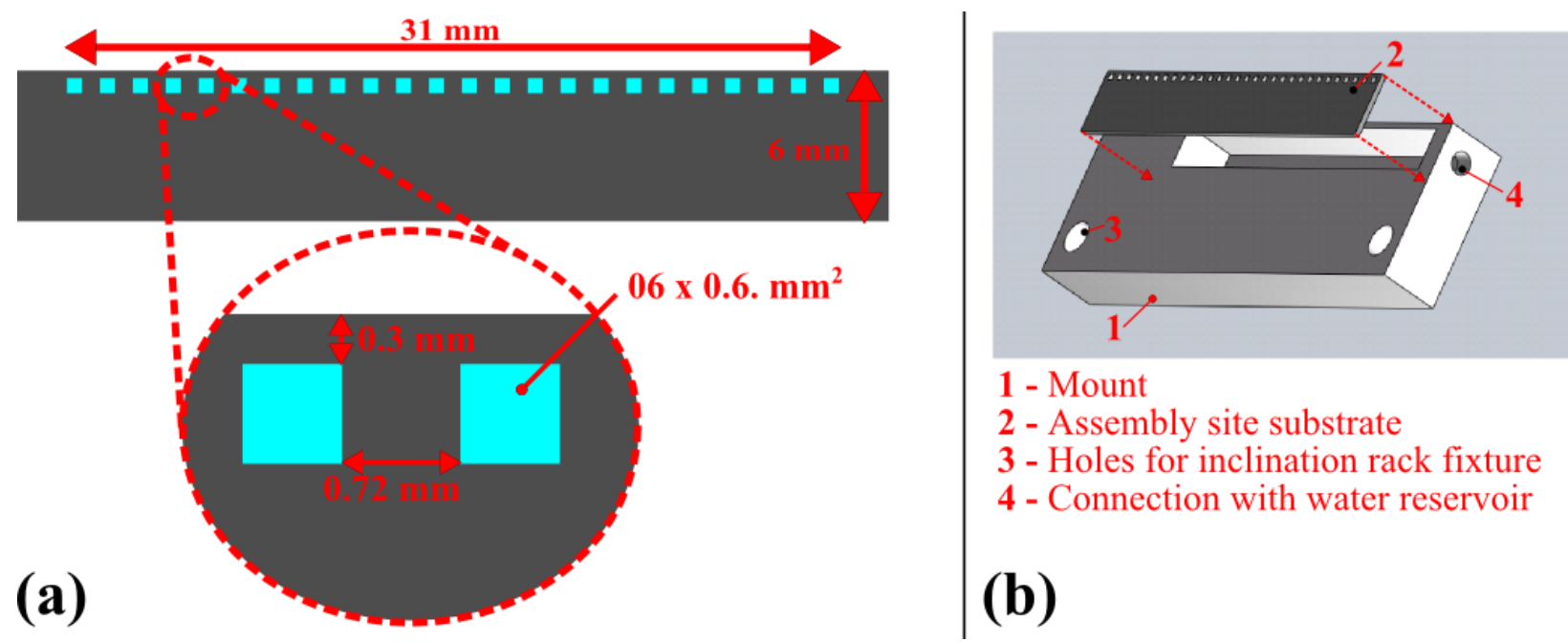

Figure 13 Geometry and mounting fixture of air-medium assembly sites. (a) Geometry of substrates with assembly sites, used for air-medium tests (drawn to scale). (b) Diagram of substrate-mount-assembly. The mounts are fixed on an inclined rack, which create the desired angle of incidence (between the substrate and the vertical motion of parts).

Next, the DRIE step lasted 40 minutes for etching all the way through the silicon wafer. Afterwards, the wafer was cleaned, diced into strips, and mounted (using glue) into an acrylic fixture. Geometry of the strips, and the acrylic mounts is shown in Figure 13b. The connection to the acrylic fixture allowed for feeding water from the elevated reservoir.

\subsubsection{Assembly Parts}

Except for the metal deposition step, fabrication of assembly parts followed the same procedure as the water-medium parts. Bare silicon wafers were diced into assembly parts, without prior metal deposition.

\subsubsection{Surface Preparations}

The parts were rendered hydrophilic using the same $\mathrm{H}_{2} \mathrm{O}_{2}$ (1-hour) treatment as that for water-medium samples. This means that $100 \%$ of the part surfaces were binding surfaces. On the other hand, the strips with assembly sites were rendered hydrophobic before mounting them to the acrylic fixture. The hydrophobic treatment was also a 24 hour solution treatment. However 
for this case, the solution was a $1 \mathrm{mM}$ octadecyltrichlorosilane (ODTS) in toluene. This SAM deposited on all $\mathrm{Si}$ and $\mathrm{SiO}_{2}$ surfaces. Hence, all surfaces of the silicon strip became hydrophobic; and provided much less bonding energy than the bonding liquid in the assembly sites.

Figure 14 Profile view of a water site in air medium. The height $\mathrm{h}$ was measured from the digital image.

After surface treatment, the strips with assembly sites were glued to the acrylic mounts (see Figure 13b). The water reservoir was then connected to the mount, so that water could fill the inside-cavity of the mount. The water level of the reservoir allowed for a positive pressure to raise the water at a desired height $h$ above the non-binding surfaces. Profile-view images allowed for measuring the site height $h$ (see Figure 14). Site-height measurements are summarized in Table 3, and the standard deviation yield a measure of how uniform the site height for several substrates.

Table 3 Assembly site height measurements for air-medium tests. For each height, 24 sites of one strip were measured.

\begin{tabular}{|c|c|c|c|}
\hline & $\begin{array}{c}\text { Average } \\
(\mu \mathrm{m})\end{array}$ & $\begin{array}{c}\text { Standard Deviation } \\
(\mu \mathrm{m})\end{array}$ & $\begin{array}{c}\text { Relative Standard } \\
\text { Deviation }(\%)\end{array}$ \\
\hline Height 1 & 60.6 & 3.4 & 5.6 \\
\hline Height 2 & 88.1 & 3.7 & 4.2 \\
\hline Height 3 & 123.0 & 5.1 & 4.2 \\
\hline Height 4 & 157.4 & 8.4 & 5.3 \\
\hline
\end{tabular}




\subsection{Part-Dropping System}

A pick-and-drop system was implemented to drop parts on top of assembly sites to create part-site interactions with controlled velocity and numbers. A gripper tool replaced the milling spindle of a $\mathrm{CNC}$ milling machine; hence converting such machine to a pick-and-drop system. This section describes how the $\mathrm{CNC}$ program was written, and how the pick-up and drop sequence was implemented for every SA test. Also, this section explains how pick-up, and drop locations were fed into the $\mathrm{CNC}$ program.

\subsubsection{CNC Program for Pick-and-Drop Sequence}

The CNC machine was programmed to run the following sequence of commands for every part:

1. Position gripper on part location, at travel height

2. Lower gripper to pick-up height (this is where part is grasped by the gripper)

3. Raise gripper back to travel height

4. Position gripper on drop location, at travel height

5. Lower gripper to drop-height.

6. Release Part
a. Turn Vacuum Off (only for vacuum gripper).
b. Turn Vacuum On (only for vacuum gripper).

7. Raise gripper back to travel height.

Steps 1 through 7 were repeated for every part that was dropped in one test run. Each test run dropped an equal fraction of the total number of parts dropped for one SA test. The travel height was set for the gripper to travel safely between part locations and drop locations. The commands were written in GCode syntax. Multiple references for learning GCode programming 
can be found elsewhere (Smid, 2008). A sample program of an actual SA test is presented in Appendix B.

Drop locations were randomly generated within a certain area over assembly sites. The size of the area was designed to ensure a uniform probability for any site to interact with a dropped part. Details about dropping-area are given in Chapters 4 and 5. The Matlab script for generating random drop locations is presented in Appendix C.

Pickup locations corresponded to where parts were located on the pick-up plate surface (see Figure 5). The following section explains how part locations where acquired. Parts were manually spread on top of the pickup plate, while ensuring that parts were at least 3-5 partlengths away from each other. The pickup plate was $5 \times 5$ in $^{2}$ made from a $1 / 4$ in thick acrylic sheet. While setting the plate on top of a light box, a top view (grayscale) image of the parts was taken using a digital imaging system (IDS UI-1460SE camera with a Computar M1214-MP2 lens, interfaced through IDS UEye Software). The image was then processed, and analyzed with Matlab (see Appendix C), so that parts were identified as objects with known XY locations (having the top-left corner of the image as coordinate system origin).

These drop locations, and part locations were fed as input to a Matlab script that outputs a file with commands (as listed above in steps 1 through 7) in GCode syntax. The CNC machine would read these files for executing SA tests. The Matlab scripts for image processing partlocation recognition, and GCode generation are all explained in Appendix C.

\subsubsection{Gripper Designs}

A capillary gripper was used for the water-medium tests, presented in Chapter 4. On the other hand, a vacuum gripper was used for air-medium tests. Figure 15 illustrates the two types of grippers. 

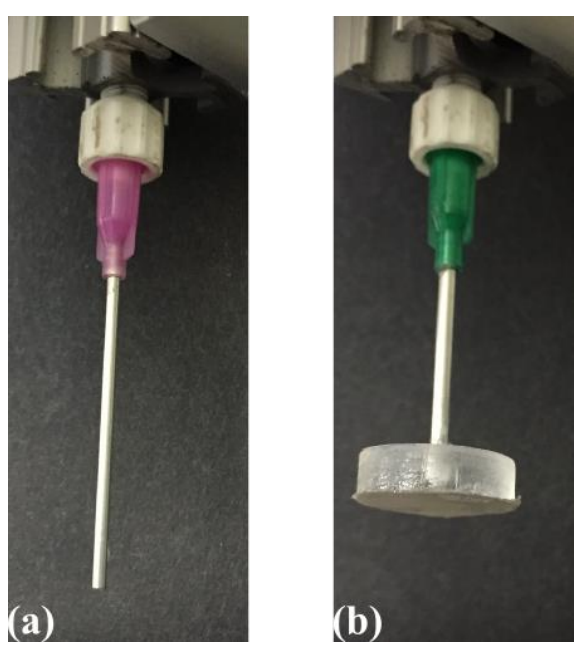

Figure 15 (a) Capillary gripper (a) and vacuum gripper (b).

\subsubsection{Capillary Gripper}

For the capillary gripper, a micrometer syringe (Gilmont GS-1100) and an 18-gauge stainless steel blunt needle were used to dispense a $1 \mu \mathrm{L}$ water droplet. More details and design considerations on capillary grippers can be found elsewhere (Gauthier \& Regnier, 2010). The hanging droplet picked up a part upon contact by the wetting forces even when wetting a hydrophobic surface. Hence, the z-axis of the $\mathrm{CNC}$ machine would move gripper downwards until the part adhered to the droplet. Precise downwards motion avoided contact between the droplet and the underlying surface. Subsequently, the part was released after plunging the needle through the water surface. The plunge-motion was performed at a high speed ( $\mathrm{Z}$-axis). Once a part was released, and the tool was lifted off the water surface, the hanging droplet self-adjusted to its original size before picking up a part. Before testing, the needle was purged to remove any air bubbles as the presence of air bubbles inside the needle or the syringe glass tubing could interfere with having a consistent liquid surface at the base of the pick-up tool. 


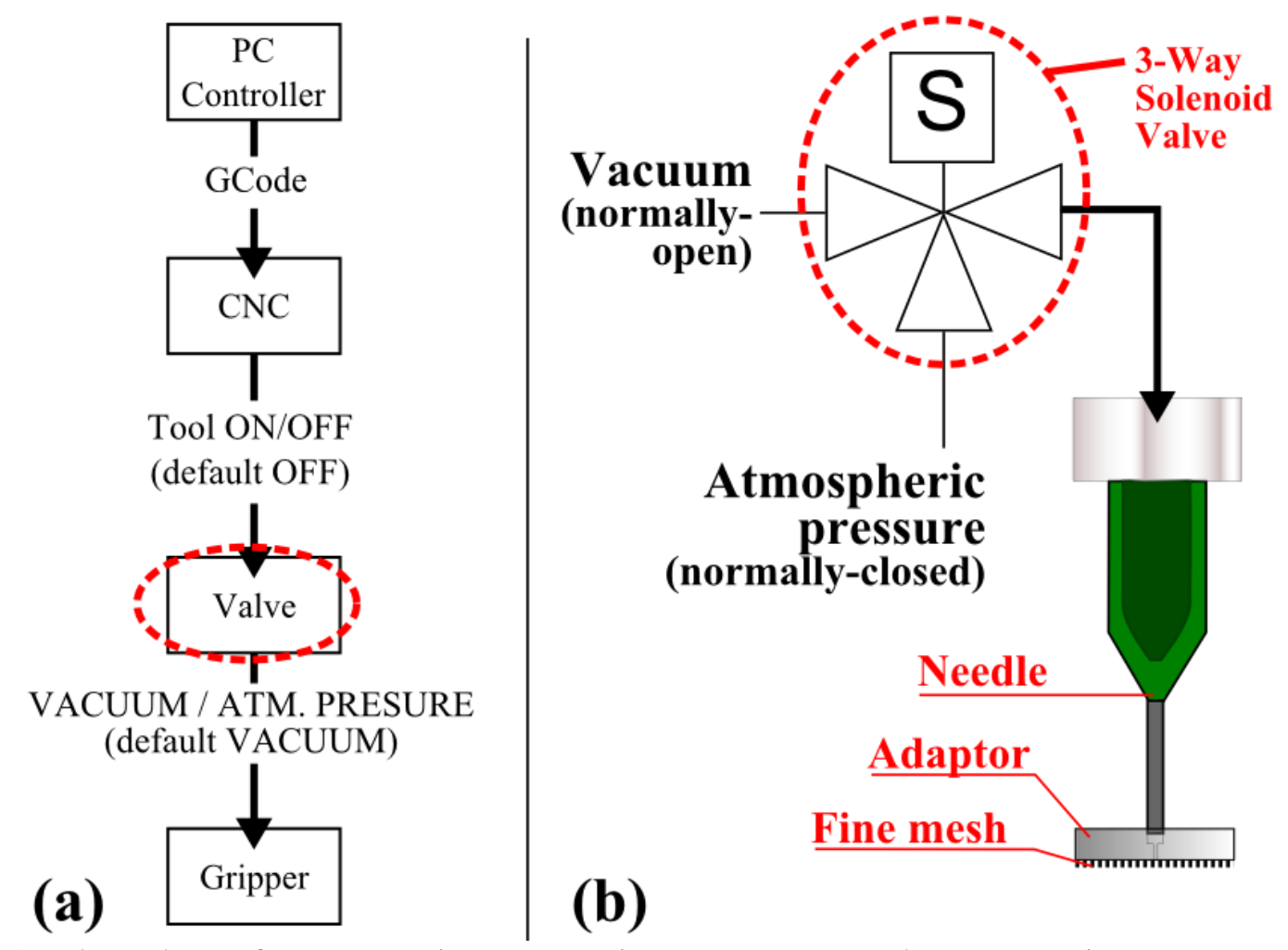

Figure 16 Flow chart of vacuum gripper actuation process (a) and vacuum gripper components (b). The CNC sends the switching command to the valve through the "Tool ON/OFF" signal.

The vacuum flows during normal operation (tool command is OFF). Part pick-up occurs when the gripper is close enough so that the vacuum "pulls" a part against the mesh. When switched $\mathrm{ON}$, the valve stops vacuum from flowing, and lets atmospheric pressure release the picked-up part.

\subsubsection{Vacuum Gripper}

The system components for actuating the vacuum gripper are illustrated in Figure 16. The tip of the gripper was fabricated by laser-cutting a 0.22 in thick acrylic sheet into a round disk, with a $0.6 \mathrm{~mm}$ hole in the center. A fine metal mesh was glued to the side to be in contact with parts. The center hole was widened on the opposite side, for pressure-fitting the tip a 14 gauge stainless steel needle. Glue was applied to ensure an air-tight seal between the acrylic and the needle. A 3-way solenoid valve (Clippard EVO-3-24-H) was connected between the vacuum supply and the gripper tool. The valve settings were switched by the tool ON/OFF signal of the CNC. Figure 16 illustrates a schematic of the vacuum, and electrical connections between all 
components required to operate the vacuum gripper. The part-grasping step (step 6-a listed above) occurred when the CNC tool command was set to OFF (default setting). This setting allowed the vacuum flow entering through the gripper tool. The gripper was lowered on top of the part, close enough until the part was sucked against the metal mesh. Next, the part was released when the tool command was set to $\mathrm{ON}$ (step 6-b listed above). This setting causes the valve to close the vacuum flow from the gripper, and instead bring the gripper tool to atmospheric pressure.

\subsection{Conclusions}

The SA interaction parameters were identified as energy ratio, part orientation and angle of incidence. The experimental system allowed for controlling each parameter independently. The present chapter reviewed the procedures for controlling each parameter. Moreover, the experimental system was designed to run tests in either water medium, or air medium tests. Water medium tests allowed for evaluating $\rho_{o}$ and $\varphi$ parameters at low $E_{k} / E_{\mathrm{b}}$ ratios. Air medium tests allowed for varying the energy ratio by controlling the drop height, thus part landing velocity. Each test configuration deserved a different sample design. Samples were fabricated through standard silicon-based processes. The test configurations, and sample fabrication methods were explained above. Furthermore, each test configuration also required a different gripper design. A capillary gripper picked and drop parts for water-medium tests, and a vacuum gripper did so for air-medium tests.

The following chapter focuses on the explanation of the parametric model which relates the afore-mentioned parameters with assembly probability. Moreover, Chapter 4 also presents the experimental evaluation of part orientation $\rho_{o}$, and $\varphi$. 


\section{CHAPTER 4:}

\section{SELF-ASSEMBLY KINETICS OF MICROSCALE COMPONENTS: A PARAMETRIC EVALUATION}

\subsection{Note to Reader}

This chapter has been previously published as an early online release article in Journal of Microelectromechanical Systems, 2014, vol. PP, no. 99, 1, and has been reproduced with permission from IEEE. Permission is included in Appendix D.

\subsection{Introduction}

Traditional assembly methods are based on a serial "grasp, position, and release" process, but this approach does not scale well to the microscale (Gauthier \& Regnier, 2010; C.J. Morris et al., 2005). Alternatively, self-assembly (SA) processes can be adapted for microscale integration (Cohn et al., 1998). SA arises from stochastic component interactions and from a balance between attractive and repulsive forces. While attractive forces provide bonding, repulsive forces prevent components from remaining in undesired configurations. Provided an adequate mobility for interacting with each other, components assemble spontaneously while also having the mobility to escape local minima in bonding energy to reach minimum energy bonding configurations. This is seen as high alignment accuracy and low errors in assembly (Pelesko, 2007). There is potential for significant cost advantages because SA systems do not require expensive manipulation systems, and feedback mechanisms (Jiandong Fang \& Böhringer, 2008; 
C.J. Morris et al., 2005; Whitesides \& Grzybowski, 2002). Moreover, high throughput is possible because parts can readily self-assemble in parallel.

Most successful microscale SA systems utilize a liquid ambient environment. A liquid medium reduces the apparent weight of components (Nathan B. Crane et al., 2013; M Mastrangeli, Abbasi, et al., 2009; C.J. Morris et al., 2005). Also, it reduces unwanted adhesion due to Van der Waals and electrostatic forces. On the other hand, the use of liquids induces challenges for microscale integration, such as avoiding corrosion, electrical contact shorting and liquid residue contamination (J. Fang \& Bohringer, 2006; Christopher J; Morris \& Parviz, 2008). Nevertheless, previous SA systems have overcome these challenges through different ways (S. E. Chung, Park, Shin, Lee, \& Kwon, 2008). These challenges are not within the scope of this study.

While SA systems have utilized various bonding-mechanisms including magnetic (Fonstad, 2002; Shet et al., 2004), electrostatic (K.-F. Bohringer et al., 1998), and fluid pressure (Tolley, Baisch, et al., 2008), capillary forces have been most widely used (C.J. Morris et al., 2005; Srinivasan et al., 2001; W. Zheng \& Jacobs, 2005). Many capillary SA systems are based on hydrophobic interactions (Srinivasan et al., 2001). Hydrophobic patterns can be created through standard micro-patterning techniques, together with selective deposition of selfassembled monolayers (SAM) (Sigma Aldrich, 2006; Whitesides \& Laibinis, 1990) and/or oxidation reactions (Onoe et al., 2004). Capillary SA based on hydrophobic interactions has been implemented successfully for assembling solar cells (RJ J Knuesel \& Jacobs, 2010), LED's on flexible substrates (Jacobs et al., 2002), thermoelectric devices (Nathan B. Crane et al., 2009), piezoelectric transducers (J. Fang et al., 2006) and MEMS devices of different batches into a 
common substrate (Liu et al., 2007). Furthermore, capillary SA has been evaluated as a feasible additive manufacturing process (N.B. Crane et al., 2011).

Successful application of SA requires high assembly rates and yield (i.e. few errors). This is especially true at the microscale, where interaction frequencies are dramatically lower relative to nanoscale processes (Nathan B. Crane et al., 2013). Maintaining adequate assembly rates requires maximizing the assembly probability of each interaction. However, at the microscale, little is known about the relationship between the physical process parameters and process rates (Srinivasan et al., 2001; W. Zheng \& Jacobs, 2005). This hinders SA commercialization.

SA processes are complex involving many bodies and multiple physical phenomena. Stochastic models have been derived from physics of reactant-interactions to consider process parameters such as relative number of reactants, geometries and densities, number of possible reactions, and relative workspace volume (Massimo Mastrangeli et al., 2010). However, these models are computationally expensive, require experimental data for calibration, and are not based on a validated model of SA interactions that could guide effective process optimization. Reaction kinetics methods provide inexpensive models of the time evolution of SA processes, and could predict changes in assembly rates if based on physical parameters. However, current formulations are based on experimental measurements unrelated to controllable process parameters (Hosokawa et al., 1994; Massimo Mastrangeli, Mermoud, \& Martinoli, 2011; Pelesko, 2007; W. Zheng \& Jacobs, 2005).

Understanding of key relationships between SA process yield and key variables such as part geometry, speed, orientation, and size enables efficient process development. This work proposes a parametric model for the most basic SA event: one part interacting with one receptor 
site. While interactions between multiple parts may play an important role in SA process rate, the present objective is to first understand how basic part-site interaction outcomes relate to measurable process parameters. Further work should then assess the role of part-part interactions, based on the findings provided here. The proposed model could enhance an event-based simulation similar to (Massimo Mastrangeli et al., 2010) or supply process-rate information to a reaction kinetics model. Such a model is meant to facilitate design and application of SA. A relationship between defined parameters and SA process rate is presented next. Subsequent section presents details on the experimental system for evaluating scaling relationships. Such system is not an actual SA system. Instead, it creates single part-site interactions under controlled conditions for measuring the impact of key process variables on assembly rate. In the last section, the authors discuss the measured effects that part geometry and part-travel direction have on SA process rate.

\subsection{Understanding SA Processes}

A basic microscale SA process behaves as a first order reaction between two reactants: unassembled parts and assembly sites (Hosokawa et al., 1994; W. Zheng \& Jacobs, 2005). When part supply is much higher than that of sites, the SA system simplifies into a pseudo first order reaction: $A \rightarrow B$; where $A$ is the number of empty assembly sites, and $B$ is the number of correct part-site assemblies. Part-site interactions can also produce a number of assemblies $(C)$ where parts are incorrectly oriented (corresponding to undesirable, yet stable energy states) represented as a competing reaction $A \rightarrow C$. The rates for these parallel, competing first order reactions are given by:

$$
\begin{array}{ll}
\dot{B}=k_{B} \cdot \mathrm{A} & \text { Equation 3 } \\
\dot{C}=k_{C} \cdot \mathrm{A} & \text { Equation } 4
\end{array}
$$


where $k_{B}$ and $k_{C}$ are the rate constants for each reaction (Houston, 2001). Maximizing the rate of assembly $(\dot{B})$ is a likely process design objective for increasing process throughput., Minimization of $\dot{C}$ should further enhance SA process yield as the incorrect assemblies can induce significant reduction of performance, as reported previously (Crane, Nathan B.; Mcknight, 2012). Both objectives (maximize $\dot{B}$ and minimize $\dot{C}$ ) are regarded as main objectives when designing a SA system (Pelesko, 2007). Full process modeling requires characterization of all reactions, but this work will focus on the primary desired reaction $(A \rightarrow B)$.

In chemical reactions, the Arrhenius equation $\left(k=A e^{-E_{a} / K T}\right)$ relates the reaction rate constant $(k)$ to the attempt frequency $(A)$, activation energy $\left(E_{a}\right)$, Boltzmann Constant $(K)$, and temperature (T). The probability of successful reactions is a function of the energy ratio $E_{d} / K T$, where KT is the average thermal energy (Houston, 2001). Self-assembly may be governed by a similar energy ratio. However, a new energy ratio definition is required because $K T$ is not an effective measure of the average kinetic energy at the microscale.

A useful kinetic model would relate assembly rate $\dot{B}$ to the probability of each part-site interaction becoming a successful assembly. Each interaction occurs when an assembly part arrives at one assembly site and its immediate surroundings. Here we consider a stationary site though in general it could be free to move. During each interaction the part position, orientation, and speed relative to the site can vary stochastically with a distribution that may not be known. Assembly occurs when the bonding energy overcomes part kinetic energy to assemble a part to a site. While different parameterizations can be applied, the ideal parameters should be easily measured and controlled during an assembly process. We propose to express the outcome of each assembly attempt in terms of four parameters: part kinetic energy $E_{k}$, nominal binding energy $E_{b}$, angle of incidence $\varphi$, and the probability $\rho_{o}$ that a part's orientation permits bonding 
(See Figure 17). The assembly probability assumes a uniform distribution in part positions.

Hence:

$$
\dot{B}=\rho_{a}\left(E_{k}, E_{b}, \rho_{o}, \varphi\right) \cdot r_{i}
$$

Equation 5

where the function $\rho_{a}$ is the probability that one attempt becomes a successful assembly; and $r_{i}$ is the rate at which assembly attempts occur. In this work, the parameter $\rho_{o}$ was directly related to part geometry (see next section). Alternative reactions such as $A \rightarrow C$ would be characterized by a similar function of the same variables. A model framework is required to test the accuracy of this parameterization scheme, and identify a suitable function $\rho_{a}$ in Equation 5.

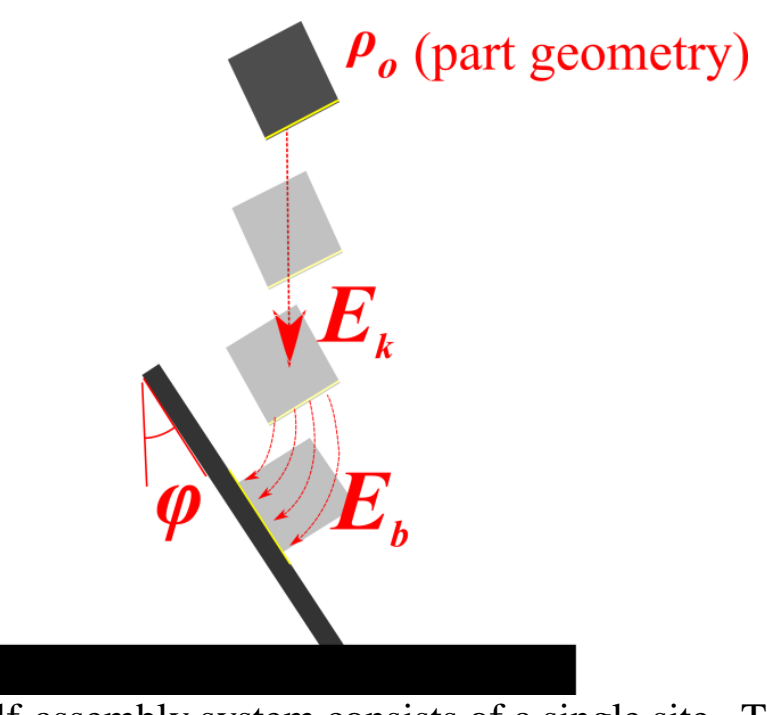

Figure 17 Unit cell of a self-assembly system consists of a single site. The process performance is determined by the outcomes of individual parts interacting with an assembly site. Key process parameters (to be evaluated) are part kinetic energy $\left(E_{k}\right)$, binding energy $\left(E_{b}\right)$, angle of incidence $(\varphi)$, and probability of correct part orientation $\left(\rho_{o}\right)$

The following hypotheses will simplify the empirical evaluation scheme for Equation 5:

1. For a purely energy-based process, zero assemblies are expected when the magnitude of kinetic energy Ek is greater than binding energy $\mathrm{Eb}$ (i.e. $\rho \mathrm{a}=0$ once $\mathrm{Ek} / \mathrm{Eb} \geq 1)$. Similarly, $\rho$ a would increase to a maximum value as $\mathrm{Ek} / \mathrm{Eb}$ decreases (while still having enough Ek for a part to travel and land on a site). Alternative 
(and possibly undesirable) assembly states associated with local minima would also vary with energy but with a different Eb value. Effects from both energy parameters could be evaluated as a function of the ratio $(\mathrm{Ek} / \mathrm{Eb})$ as is done in chemical reactions. Condensing energy effects into the ratio Ek/Eb may neglect non-linear effects such as impact that will be more pronounced at higher energies values (even without varying Ek/Eb ). However, given the limited data on SA process modeling, it is reasonable to start with this simplest assumption and refine where necessary based on additional data. These tests utilize interactions at very low Ek/Eb ratios, minimizing (hence, neglecting) non-linear effects. This is the area of greatest practical interest as assembly probabilities are highest in these regions, Effects of varying Ek/Eb, as well as those of impact and viscoelastic forces will be addressed in a future work.

2. If SA is a purely energy-based process, then assemblies would be independent of the assembly path and thus independent of the angle $\varphi$ at which parts arrive on sites. This paper reports the results of tests to evaluate this hypothesis. In order to test path independence, the effects of $\varphi$ on the initial assembly rate is measured as a single-variable function $\mathrm{g}(\varphi)$.

3. When a part has zero probability of being correctly oriented (i.e. $\rho$ o $=0$ ), then $\rho a$ $=0$. Similarly, $\rho o=1$ (i.e. a part that can bond correctly regardless of its orientation) would increase $\rho$ a to its possible maximum. It is also reasonable to expect a linear relationship between $\dot{\mathrm{B}}$ and $\rho$.

Accordingly, Equation 5 is restated as

$$
\dot{B}=g(\varphi) \cdot f\left(E_{k} / E_{b}\right) \cdot \rho_{o} \cdot r_{i} \quad \text { Equation } 6
$$


The above postulates will be tested by experiments as they provide a framework for evaluating the key SA process parameters $\left(E_{K}, E_{B}, \rho_{o}\right.$ and $\left.\varphi\right)$. The remainder of this work will cover: 1$)$ an experimental system for measuring SA rate while controlling each SA parameter individually; and 2) empirical evaluation of function $g(\varphi)$ and the effects of $\rho_{o}$. Tests for this work were performed under the condition $E_{K} / E_{B}<<1$. Hence, the above stated postulate \#1 allows for simplifying Equation 6 to $\dot{B}=g(\varphi) \cdot \rho_{o} \cdot r_{i}$ and thus for simpler evaluation of $\rho_{o}$ and $g(\varphi)$ (postulates 2 and 3). Future work will focus on evaluating the function $f\left(E_{k} / E_{b}\right)$,

\subsubsection{Experimental SA System}

\subsubsection{Experimental Procedure}

The objective of our experimental system was to measure assembly rates $\dot{B}$ and $\dot{C}$. For this purpose, the system was designed for creating sequential assembly attempts by dropping assembly parts, one-by-one on an area filled with fixed assembly sites, while controlling the parameters $E_{k}, E_{b}, \varphi$, and $\rho_{o}$. For each test, assemblies were observed, and counted after regular increments of parts dropped. Thereafter, the numbers of correct and incorrect assemblies could then be plotted against the number $n$ of parts dropped (i.e. $B(n)$ and $C(n)$ respectively). Integrated forms of Equation 3 and Equation 4 yields the assembly states $B(n)$ and $C(n)$ (Houston, 2001). These are parallel first order reactions, meaning that the rate at which unfilled assembly sites decrease is $-\left(k_{B}+k_{C}\right) \cdot A(n)$ where $n$ is the unit of time; i.e. the process progressed with every part dropped. $A_{o}$ is the number of empty assembly sites at $n=0$ parts dropped. Accordingly,

$$
\begin{array}{ll}
\frac{B(n)}{A_{\mathrm{o}}}=\frac{k_{B}}{k_{B}+k_{C}} \cdot\left(1-e^{-\left(k_{B}+k_{C}\right) \cdot n}\right) & \text { Equation } 7 \\
\frac{C(n)}{A_{\mathrm{o}}}=\frac{k_{C}}{k_{B}+k_{C}} \cdot\left(1-e^{-\left(k_{B}+k_{C}\right) \cdot n}\right) & \text { Equation } 8
\end{array}
$$


The assembly state versus number of parts dropped $(B(n)$ and $C(n))$ used to find the rate constants $k_{b}$ and $k_{c}$ were found through regression fitting. These constants were then used to calculate the initial assembly rates $\dot{B}$ and $\dot{C}$ (using Equation 3and Equation 4) for each SA test conducted.

(a)
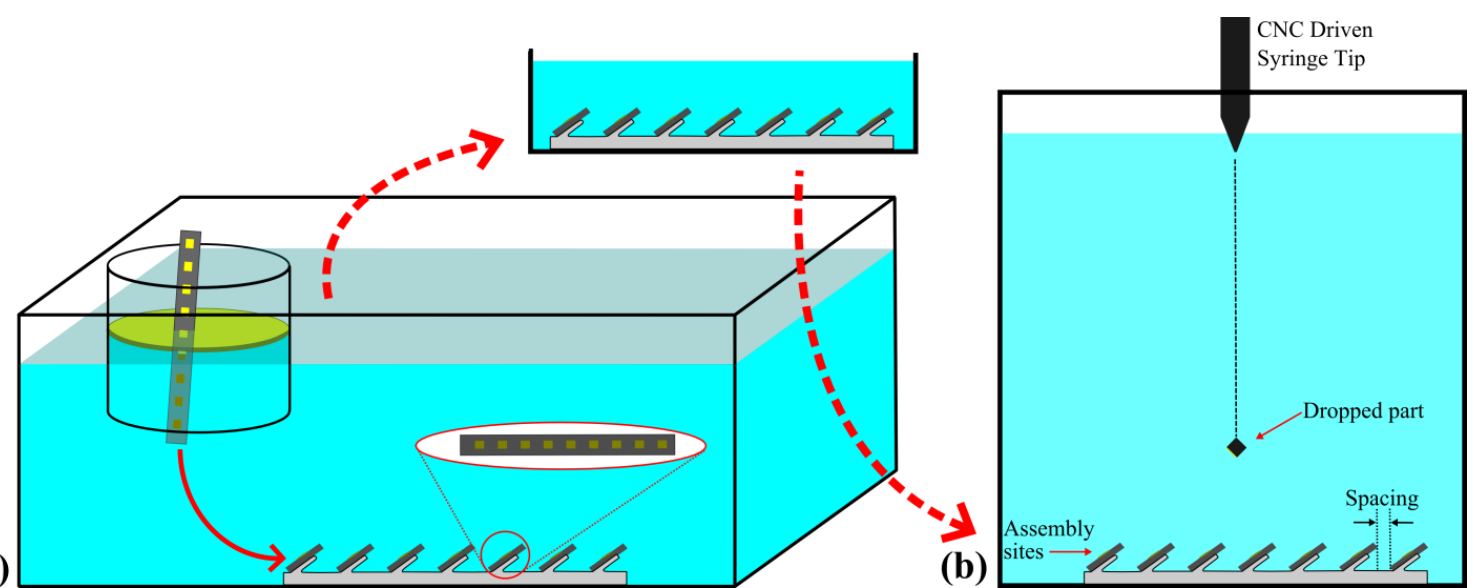

Figure 18 (a) Process for preparing assembly sites. After preparing the assembly site surfaces (oxidizing the silicon and applying a self-assembled monolayer to the gold assembly sites) the assembly sites were coated with oil by passing them through an oil film as described by Biebuyck and Whitesides (Biebuyck \& Whitesides, 1994). Sites are placed in a fixture and transported to the assembly location submerged in water. (b) Illustration of part dropping process during an SA test. Between 600 and 700 parts were dropped individually for each test.

The experimental SA system and procedure are depicted in Figure 18. The oil-deposition procedure depicted in Figure 18 was performed at repeatable speed and strip-orientation. This allowed for keeping oil-deposition as uniform as possible (Biebuyck \& Whitesides, 1994). Parts were dropped through a water medium to impact stationary assembly sites. A uniform distribution of dropped parts was achieved using a pick and place tool with a capillary gripper and randomized dropping locations distributed with a uniform probability distribution over the entire area containing assembly sites (Figure 18b). Several hundred parts were dropped in each test to ensure an adequate fitting of Equation 7 and Equation 8 to the data from each SA test. Number of assemblies $B$ and $C$ were counted by visual identification after regular increments of 
$n$ parts dropped. Figure 19 demonstrates how data in $B(n) / A_{o}$ and $C(n) / A_{\mathrm{o}}$ (for one test configuration) lies within the linear range of the exponential reaction kinetics model (Equation 7 and Equation 8),

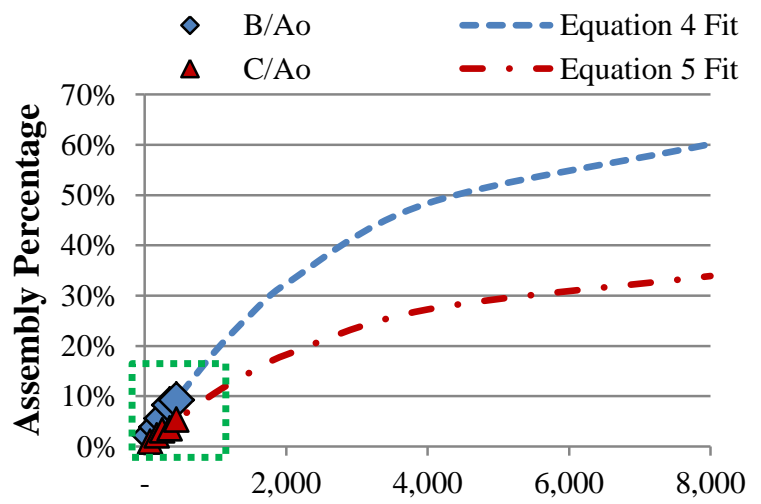

(a)

\# parts dropped (n) (b)

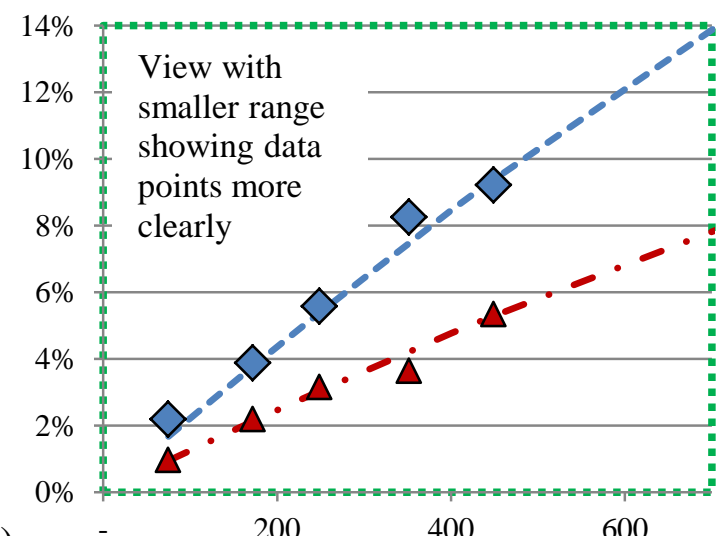

200

400

600

Figure 19 Sample chart showing assembly increments during one SA test (using 0.5 x 0.5 x 0.4 $\mathrm{mm}^{3}$ parts, and $\varphi=45^{\circ}$ ). Data points for $B / \mathrm{A}_{\mathrm{o}}$ and $\mathrm{C} / \mathrm{A}_{\mathrm{o}}$ are plotted with respect to $n$ (shown more closely in the close-up plot on the right). Data sets $B / A_{o}$ and $C / A_{o}$ were fitted into Equation 7 and Equation 8 (lines show best fit results) in order to extract $k_{B}$ and $k_{C}$ and calculate $\dot{B}$ and $\dot{C}$ through Equation 3 and Equation 4.

Standard micro-fabrication techniques were implemented for creating assembly sites and parts out of silicon substrates (photo-lithography, DRIE, and PVD of thin metal films). Although sites and parts were not actual working devices, geometry and material composition correspond to those of common microscale devices. Assembly sites were designed as $600 \mu \mathrm{m}$ x $600 \mu \mathrm{m}$ surface pads, patterned on $40 \mathrm{~mm} \times 2 \mathrm{~mm} \times 0.5 \mathrm{~mm}$ silicon strips and distributed over a total assembly area of approximately $50 \mathrm{~mm}$ x $30 \mathrm{~mm}$ (see Figure 18a). In order to focus on primary assembly effects, the strip configuration permitted for non-assembled parts to fall through and not interfere with subsequently arriving parts. Additionally, edge-to-edge spacing between adjacent sites was at least $1 \mathrm{~mm}$, preventing any part from simultaneously interacting with two adjacent sites. Each assembly site was recessed by $15 \mu \mathrm{m}$ to improve oil deposition on sites (see following section) (M Mastrangeli, Ruythooren, et al., 2009). Assembly parts were prepared by 
dicing silicon wafers with varied heights into $500 \mu \mathrm{m}$ x $500 \mu \mathrm{m}$ dies. Before dicing, wafers were coated with gold on one or both sides. The binding surfaces of sites were slightly larger than those of parts, preventing parts from interfering with the non-binding surfaces due to small misalignments.

\subsubsection{Controlling SA Parameters}

\subsection{Kinetic Energy}

The kinetic energy $\left(E_{k}\right)$ of incoming parts was controlled by dropping them at their terminal velocity under gravity through the liquid medium. This ensures that all parts arrive at the region of assembly sites with the same $E_{k}$ value. Hence, $E_{k}$ becomes a function of part density, part size, ambient liquid density and viscosity. For this work, water at $22^{\circ} \mathrm{C}$ was used as ambient liquid. Terminal velocity $(V)$ of the parts (measured from videos taken at 75 frames per seconds) was $73.6 \mathrm{~mm} / \mathrm{s}$ for $0.5 \times 0.5 \times 0.4 \mathrm{~mm}^{3}$ and $81.9 \mathrm{~mm} / \mathrm{s}$ for $0.5 \times 0.5 \times 0.5 \mathrm{~mm}^{3}$ parts

(yielding $E_{k}=6.30 \cdot 10^{-7} \mathrm{~mJ}$ and $9.76 \cdot 10^{-7} \mathrm{~mJ}$, respectively). Videos of falling parts indicated that rotational motion was negligible, and that dropped parts achieved terminal velocity after falling $5 \mathrm{~cm}$. During testing, parts fell over $12 \mathrm{~cm}$ before impact.

\subsection{Binding Energy}

Energy minimization drives self-assembly bonding. For the present work, surface energies are the primary bonding energy. Surfaces are prepared following the approach of Srinivasan et al. (Srinivasan et al., 2001). In essence, (while in water medium) contact between binding (hydrophobic) surfaces achieves a smaller energy state, than when a non-binding (hydrophilic) surface is involved. The bonding energy was calculated

$$
E_{b}=\left(\gamma_{p s}-\gamma_{s m}-\gamma_{p m}\right) \cdot a_{b}
$$


where $a_{b}$ is the binding surface area of a part, $\gamma_{p s}, \gamma_{s m}$ and $\gamma_{p m}$ are the interfacial energies, involving parts binding surface $(p)$, sites binding surface $(s)$, and water medium $(m)$. More detail about all surface compositions is given in Figure 20. The surface energy term $\gamma_{s m}$ was measured as $50.8 \mathrm{~mJ} / \mathrm{m}^{2}$ with a Du-Noüy ring tensiometer. The term $\left(\gamma_{p s}-\gamma_{p m}\right)$ was obtained via contact angle $(\theta)$ measurements and use of Young's Equation $\gamma_{s m} \cdot \cos (\theta)=-\left(\gamma_{p s}-\gamma_{p m}\right)$.For $E_{b}$ of a correct assembly (a part binding on its hydrophobic gold surface), a hexadecane drop (composition of surface $s$ ) on hydrophobic-rendered gold (corresponding to surface $p$ ), in water medium ( $m$ ) was measured as $\theta=1.94^{\circ}$ (receding angle) (Onoe et al., 2004). On the other hand, the same drop liquid and medium was used on a hydrophilic $\mathrm{SiO}_{2}$ surface to measure $\theta=85.46^{\circ}$ (receding angle), corresponding to an incorrect assembly. Accordingly, $E_{b}$ for correct and incorrect assemblies were calculated as $-22.54 \mathrm{~nJ}$ and $-13.7 \mathrm{~nJ}$, respectively, when considering the geometries implemented in the present experiments (i.e. $a_{b}=0.5 \times 0.5 \mathrm{~mm}^{2}$ ). The correct assembly $E_{b}$ value yields $E_{k} / E_{b}$ ratios between 0.028 and 0.044 (varying with $E_{k}$ for different part geometries) which agrees with the assumption made in the previous section.

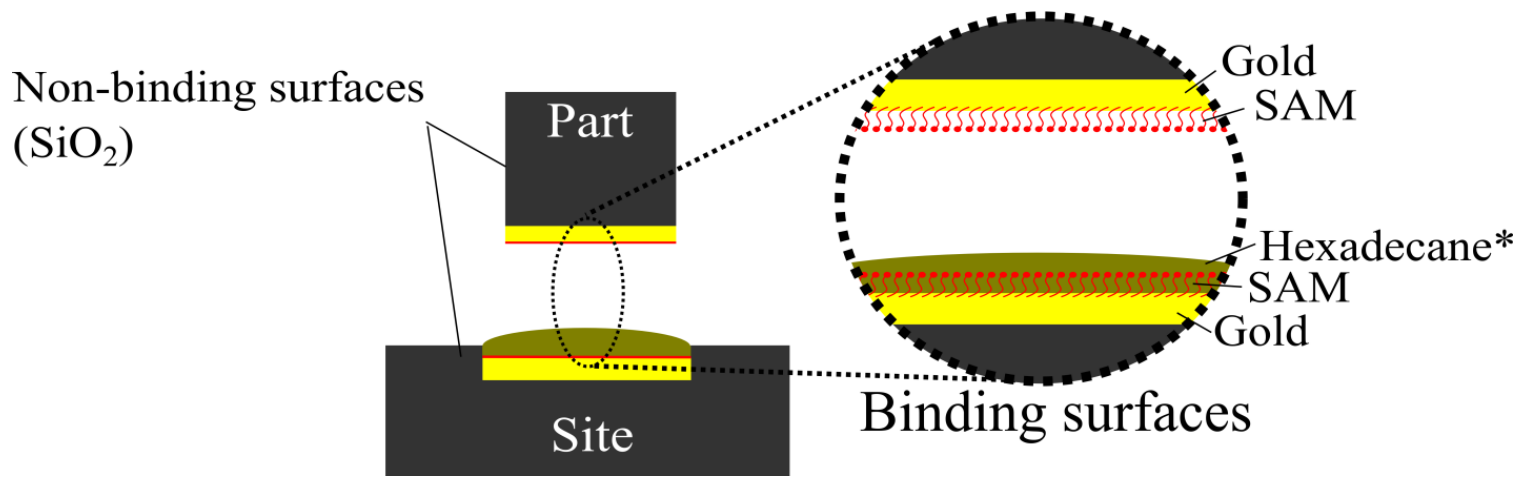

Figure 20 Surface compositions of binding and non-binding surfaces in assembly parts and sites.

The bonding liquid layer achieves two purposes: 1) provides interfacial tension desired for bonding; and 2) acts as a low friction interface that facilitates correction of initial misalignment of an arriving part. The oil/surface forces can improve both rotational and 
translational alignment(Arutinov et al., 2013; Berthier, Brakke, Grossi, Sanchez, \& Di Cioccio, 2010). While the thin liquid layer possess a certain maximum height $(\sim 30 \mu \mathrm{m})$, this is a negligible quantity for binding energy calculations. Such three-dimensionality of the bonding liquid enhances the reach of alignment forces (i.e., parts could land with certain offset tolerance and still assemble). Effects of these alignment forces are discussed below, along with experimental results.

\subsection{Angle of Incidence}

Motion of parts was determined by gravity. Therefore, incidence angle $\varphi$ was controlled by mounting the strips with assembly sites on a rack mount with desired inclination. Angle $\varphi$ was varied from $30^{\circ}$ to $60^{\circ}$, in $7.5^{\circ}$ increments.

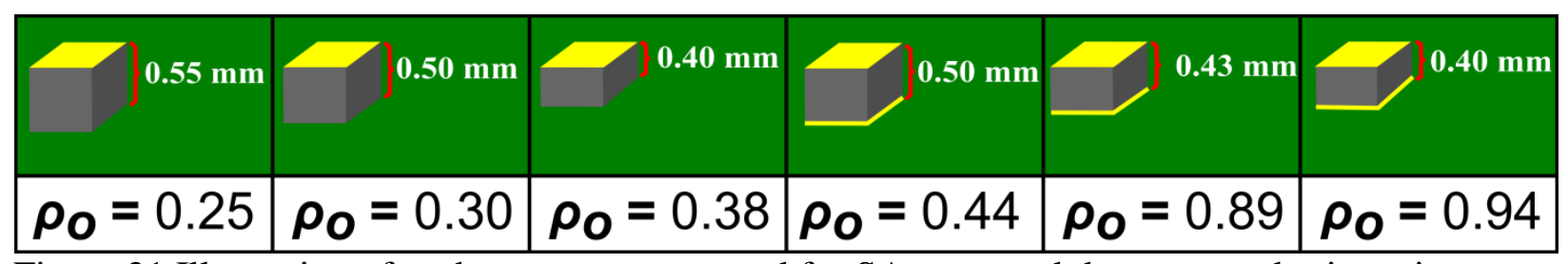

Figure 21 Illustration of each part geometry used for SA tests, and the measured orientation probability $\left(\rho_{o}\right)$ values. All parts were $0.5 \times 0.5 \mathrm{~mm}^{2}$ wide, and with variable height as illustrated.

\subsection{Orientation Probability}

While part orientation was not directly controlled, the probability of the binding surface facing downwards varied with the part aspect ratio and the number of binding surfaces. For each part configuration (depicted in Figure 21), the probability $\rho_{o}$ was measured by utilizing the pick and place process to drop approximately 300 parts on a $30 \mathrm{~mm}$ x $50 \mathrm{~mm}$ flat area, and counting how many parts landed on their binding surface (i.e. gold side down). As a consistency measure, $\rho_{o}$ measurements were repeated 3 times for every part configuration. Results are depicted in 
Figure 21. Assembly tests were then performed for different part geometries, in order to plot measured assembly rate $\dot{B}$ against $\rho_{o}$.

\subsection{Results and Discussion}

Images confirmed the existence of both assembly types ( $B$ and $C$ ) in every SA test conducted (see Figure 22). It is important to note that incorrect assemblies existed due to weaker, yet somewhat stable bonds with non-binding surfaces. Correct assemblies were more dominant than incorrect ones for every configuration tested (i.e. $\dot{B}>\dot{C}$ ). This was predicted by difference in $E_{b}$ values reported above; and is desired for efficiently evaluating the relationship between $\dot{B}$ and the key process parameters.

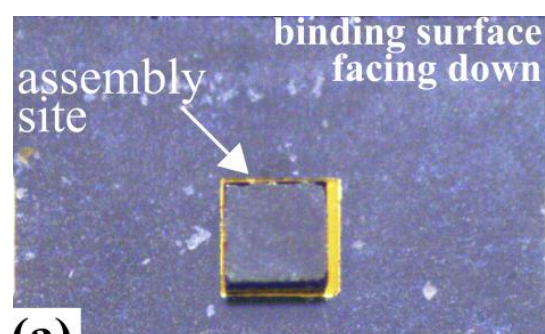

(a)

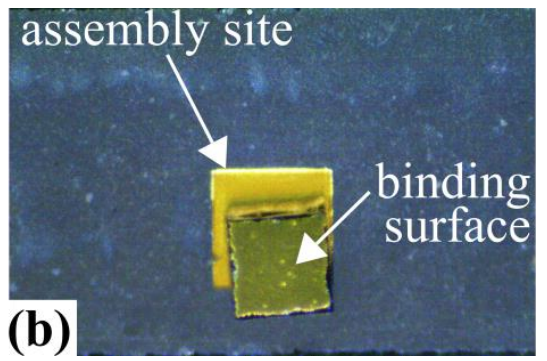

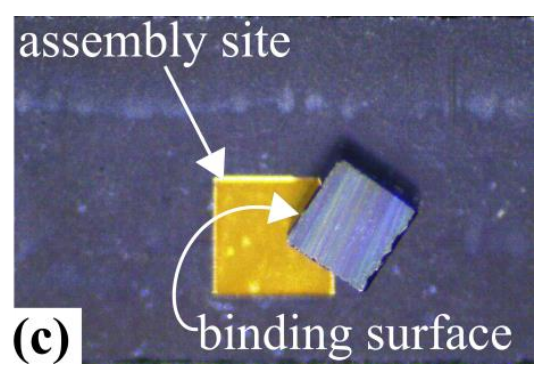

(c) binding surface

Figure 22 Correct and incorrect assembly states. (a) Correct assembly (type B) with binding (gold) surface facing downwards. (b) Incorrect assemblies (type C) with part lying on its back side. (c) Incorrect assemblies (type C) with part lying on its side.

The effects from varying part geometry and angle of incidence are shown in Figure 23. The y-axis represents the quantity $\dot{B} / r_{i}$, (adapted from Equation 6). In our case where the unit of time is one part dropped $(n)$, the rate of interaction $r_{i}$ corresponds to the probability of each part landing on a site. Hence,

$$
r_{i}=\frac{n_{\text {sites }} \cdot A_{\text {site }} \cdot \sin (\varphi)}{A_{\text {total }}}
$$

where $n_{\text {sites }}$ is the number of assembly sites, $A_{\text {site }} \cdot \sin (\varphi)$ is the projected area of each assembly site, and $A_{\text {total }}$ is the total area on which any part could possibly land. Plotting $\dot{B} / r_{i}$ normalizes 
assembly rate by changes in $n_{\text {sites }}$ and $\varphi$; hence, allowing for direct comparison between SA tests. Each data point represents that average of at least 3 tests with 500-700 parts dropped per test.

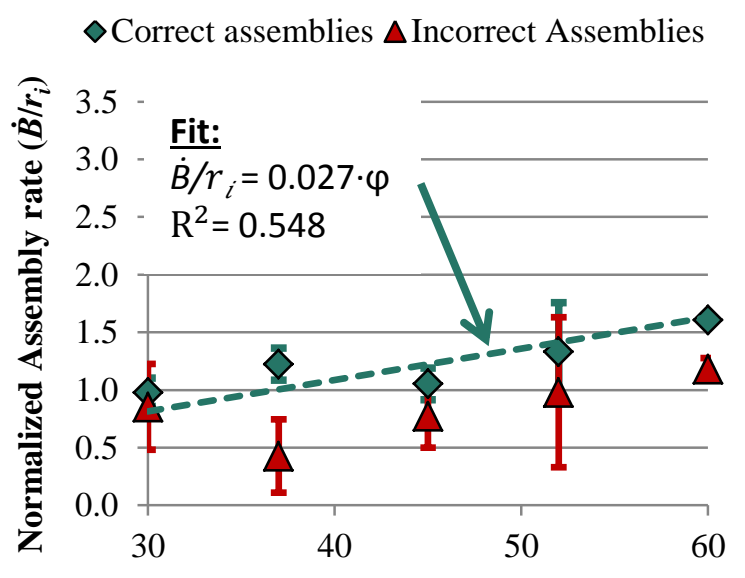

(a) (b)

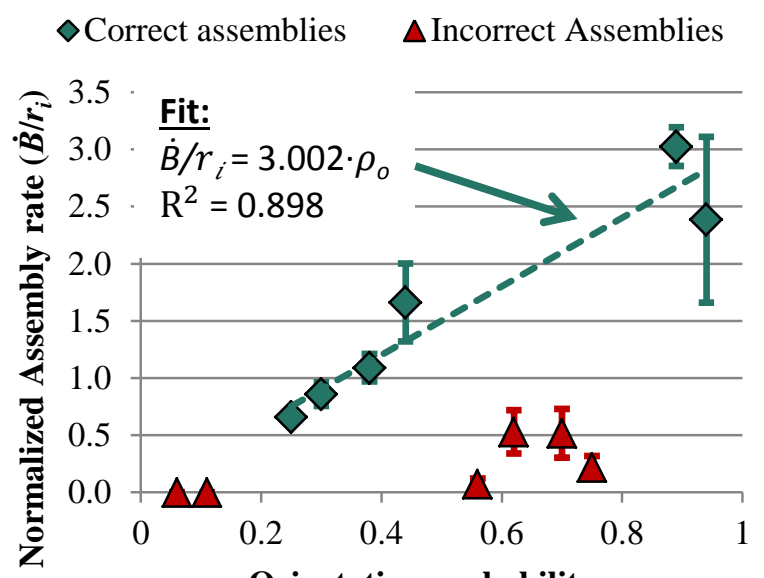

$\diamond$ Correct assemblies $\quad \Delta$ Incorrect Assemblies

Orientation probability $\rho_{o}$

Figure 23 SA rate results. a) Variation of assembly rate with incidence angle $(\varphi)$ for $0.5 \times 0.5$ $\mathrm{x} 0.5 \mathrm{~mm}^{3}$ parts offering a value $\rho_{o}=0.3$. (b) Variation of assembly rate with part orientation probability $\left(\rho_{o}\right)$ for a constant incidence angle $\varphi=45^{\circ}$. Each data point with error bars corresponds to an average, and standard deviation resulting from several SA tests, under same conditions.

Figure 23 illustrates effects of parameters $\varphi$ and $\rho_{o}$ on both assembly types. Effects on incorrect assemblies measured lower than what is measurable with the implemented experimental system. Consequently, it is difficult to draw definitive conclusions on incorrect assemblies. Nevertheless, we can observe that the incorrect assemblies follow similar trends as the correct assemblies. The main objective of the present experiments is to evaluate parameter effects on rate correct assemblies $(\dot{B})$ and only these results will be discussed below.

Figure 23-a shows that the effects of $\varphi$ are not significant -or at least are not significant relative to the experimental variation. Low effects of angle $\varphi$ would indicate that to first order, $g(\varphi)=1$ For the low $E_{k} / E_{b}$ values tested here, this would say that at the low kinetic energy, the probability of assembly is equal to the probability of the center of a part impacting over an assembly site such that $\dot{B} / r_{i}=\rho_{o}$. However, this is clearly an underestimate of the assembly 
probability for low energy conditions as even a small overlap between the part and the binding site can form a bond that would overcome the kinetic energy of the falling part. In fact, the $\dot{B} / r_{i}$ vs. $\rho_{o}$ data (Figure 23-b) is reasonably fitted by the line (that goes through the origin) $\dot{B} / r_{i}=$ $3.002 \cdot \rho_{o}$. While this agrees with the form of the hypothesized relationship in Equation 6 the rates increase with a slope $3 x$ higher than expected. Direct observation of part site interactions (see Figure 24) allowed for understanding why assembly rates measured higher than expected.

(a)
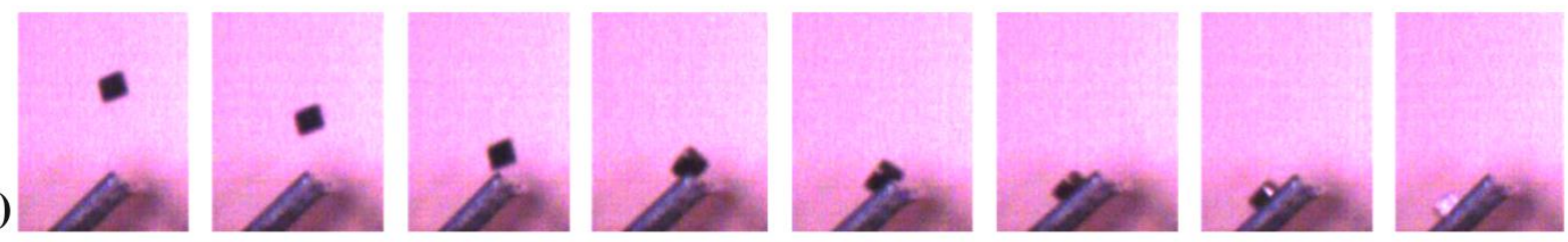

(b)
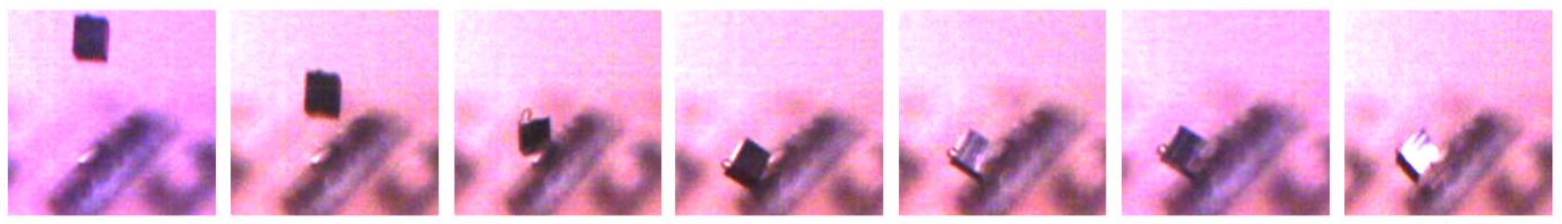

Figure 24 Image sequence of parts contacting an assembly site. (a) A part falling right above an assembly site. It then slides down and assembles directly.(b) A part moves below the assembly site but is then aligned by means of capillary forces, provided by the bonding liquid.

These observed interactions evidenced how assemblies occur even when part center of gravity impacts at an offset from assembly sites. In Figure 24 (a) the part impacted near the top of the assembly strip, but then slid down the strip and successfully assembles. In contrast, Figure 24 (b) shows a part landing well below the assembly strip, but then moved upwards onto the assembly site. Tolerance to offset misalignment is attributed to two reasons: 1) capillary interactions between bonding liquid and assembly parts generate "pull-in" forces" (described elsewhere (Arutinov et al., 2013; Berthier et al., 2010)); and 2) parts that land above assembly sites, interacting first with the non-binding surface, and then slide down into a correct assembly. At low $E_{b} / E_{k}$ ratios (such as the case in this work) effects from pull-in forces are expected to be 
stronger than at higher ratios. Secondary interactions are likely important at all value of the energy ratio.
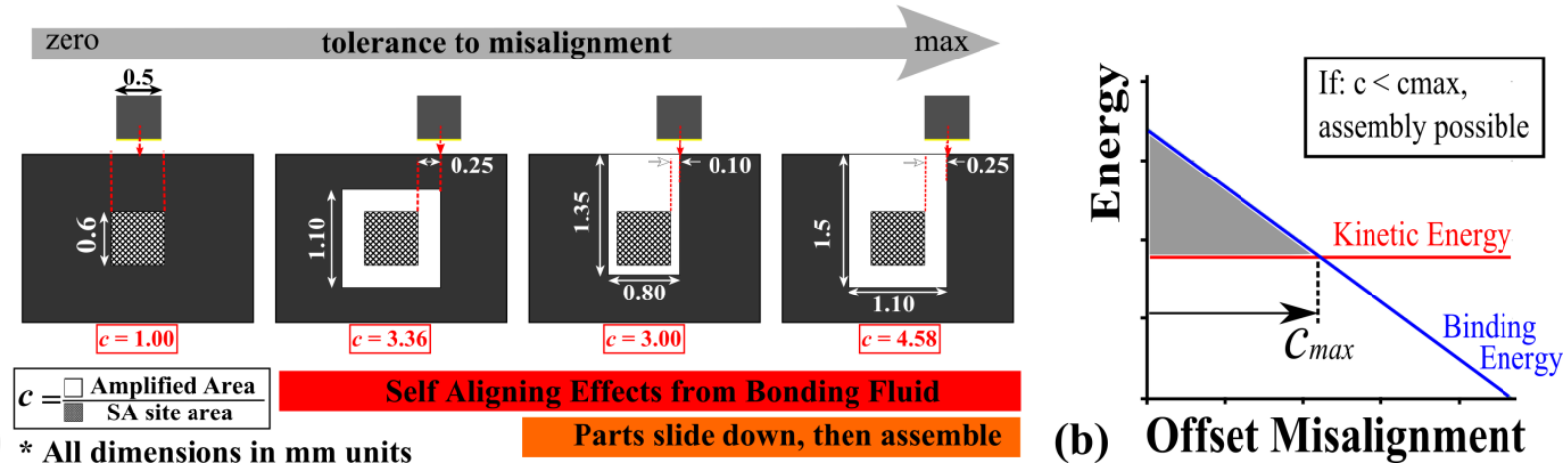

Figure 25 (a) Different levels of hypothesized misalignments are illustrated, arranged in order from minimum to maximum possible misalignment (from left to right). The white area represents where a part-centroid can arrive and still provide an assembly. The ratio $c$ quantifies the apparent increase in assembly area beyond the projected area of the binding sites. Thus, it represents the misalignment tolerance. The two right-most illustrations correspond to the cases where parts land above the site, and still assemble. (b) Illustrated relation between energies and offset misalignment, as quantified by ratio $c$. As misalignment increases, contact area between binding surfaces decreases, hence $E_{b}$ decreases. Accordingly, magnitude of $E_{k}$ at impact determines the maximum allowed misalignment.

The simplest treatment of this assembly process is to treat the observed tolerance to pullin forces and secondary interactions, as an apparent increase in assembly area. The ratio of the amplified area over the original assembly site area was applied as a correction factor $c$; thus, modifying Equation 6 to $\dot{B}=c \cdot \rho_{o} \cdot r_{i}$ (when $g(\varphi)=1$ ). As illustrated in Figure 25a, the size of such correction factor could reasonably vary from 1 (assembly occurs only when the center of gravity impacts the site) up to 4.58 (assembly occurs when any part of the binding surface touches the site or lands above the site). As stated above, an increased area ratio such that $c=3$ lies within this expected range and agrees with the linear fit shown in Figure 23-a. However, the slope of such relation is unknown for different energy levels. Offset misalignments reduce contact area between binding surfaces; thus reducing the initial binding energy (see Figure 25b). Consequently, larger $E_{k} / E_{b}$ ratios are expected to reduce the average tolerable misalignment. 
More insight into these energy effects will be obtained from studying the effects of the parameter $E_{k} / E_{b}$ (upon future work).

In order to confirm the effects of these secondary interactions on assembly rate, SA rate measurements (same procedure as those presented in Figure 23) were taken while varying the amount of space available for parts to slide. Figure 26 shows the two assembly site configurations used with varying space above sites. Results for SA tests using such sites, and cubic parts of $0.5 \mathrm{~mm}$ sides, with 1 binding surface $\left(\rho_{o}=0.3\right)$ and 2 binding surfaces $\left(\rho_{o}=0.4\right)$, confirm that assembly rate varies with the change in sliding space available. Each site configuration is well-approximated by a linear relationship between the orientation probability and the assembly rate as seen in Figure 23. However, we cannot directly predict the slope with the available space above the site. The raised sites had a negligible impact on the assembly probability while the lowered sites showed a very large effect. This is likely a result of the fact that the probability of successful assembly is dependent on non-modeled effects such as part reorientation upon impact with the substrate. When the sites are lower, a larger number of the parts are able to reorient so that the downward face is parallel to the substrate. This will facilitate assembly. The orientation probability in these cases is probably more representative of the orientation probability measurements than when the samples are impacting near the corners. It may be that the details of the part rotation on impact have a substantial impact on the assembly probability when the sites are near the top edge. For example, higher sites may compensate for having less assembly area by making contact between the oil and the part on more surfaces. When a corner hits the sites, this could improve the assembly since the oil can contact up to three surfaces simultaneously_improving the odds that one of the surfaces is an assembly surface. This could create competing effects that reduce the impact of moving the sites upward as 
observed in these tests. Further work is needed to understand the effects of these details and other issues such as the quantity (height) of the oil film on a binding site.
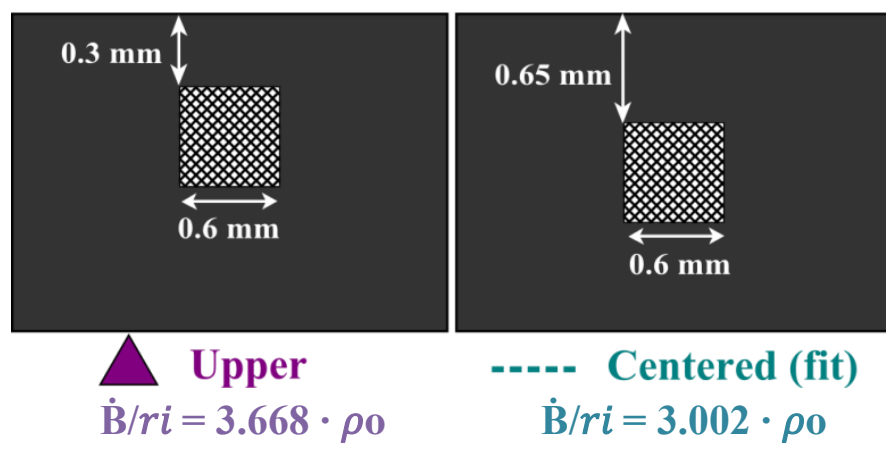
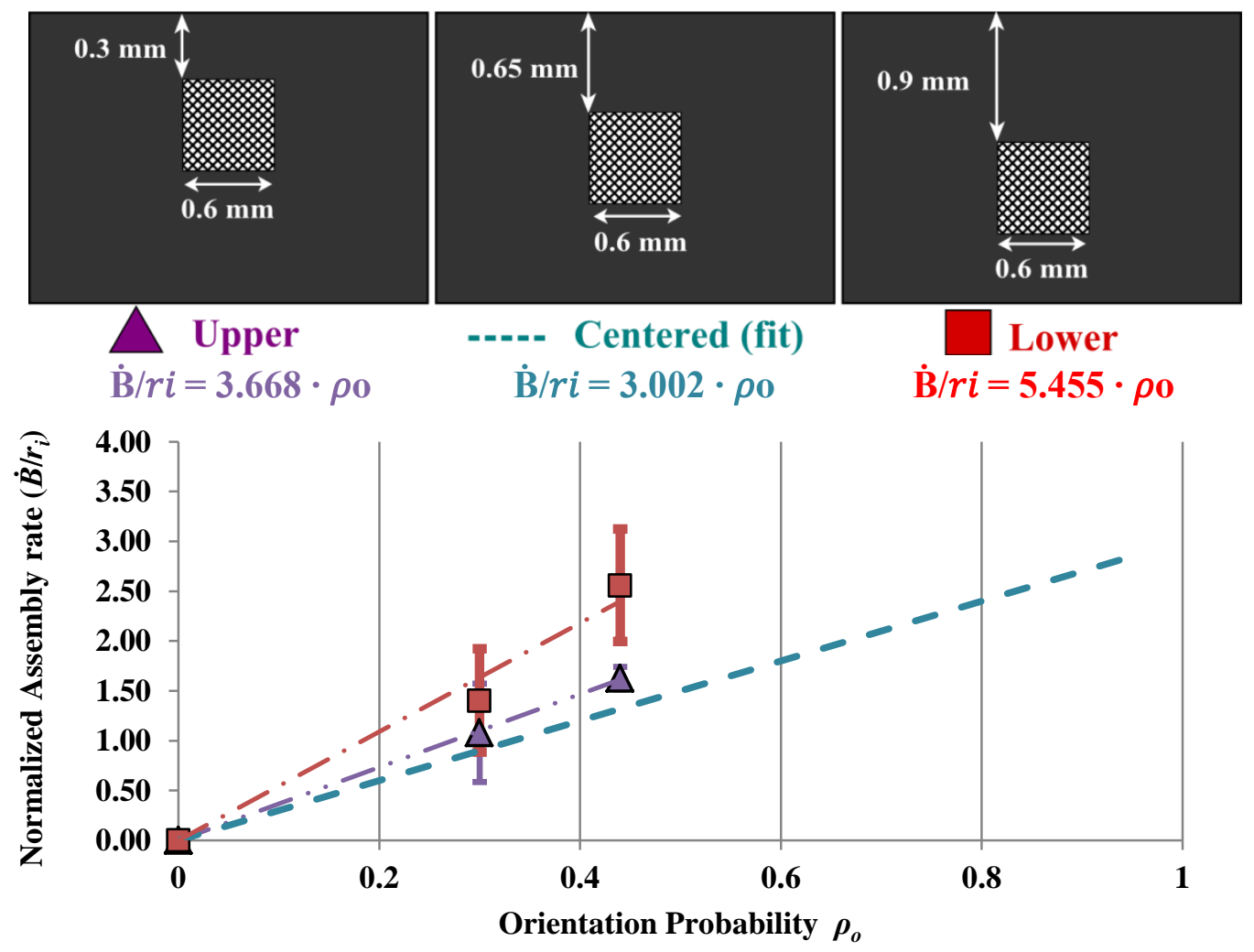

Figure 26 Assembly sites SA rate vs. $\rho_{o}$ were measured for assembly sites with varied overlaying space. While slopes do not scale with the available space above the sites, these measurements confirm that the change in such space is a clear effect on assembly rates.

\subsection{Conclusions}

A SA model based on chemical kinetics has been proposed that predicts the assembly probability resulting from an individual part-site interaction. While chemical reactions and selfassembly are analogous in many respects, SA admits to the control of many process parameters like energy distribution and part orientation in ways that are not possible in chemical reactions. This provides valuable control to overcome the slower interaction frequencies at the microscale, but it also introduces additional parameters into rate models. Four measurable parameters have been identified as potentially important: $\varphi, \rho_{o}, E_{b}$, and $E_{k}$. An experimental system is presented 
for controlling these key parameters in order to test the nature of the process relationships. The results presented here provide support for several key aspects of the proposed model, evaluating the effects of incident angle $(\varphi)$ and orientation probability $\left(\rho_{o}\right)$. These controlled experiments provide an unprecedented look at the part-site interactions in capillary self-assembly.

Results show that part geometry can be related to assembly rate linearly with the part orientation probability $\rho_{o}$. The orientation probability is a key parameter which should be improved in any SA process. While this was accomplished by changing the thickness of the parts, and rendering one, or multiple surfaces as binding surfaces, other options are possible. In other experiments performed by the authors, a (100 $\mu$ m thick) coating of SU-8 (Microchem) photoresist significantly altered the part orientation probability from $30 \%$, to $90 \%$, because the lower density of the photoresist shifted the part center of gravity. Other approaches could include magnetic or electric fields (K.-F. Bohringer et al., 1998).

SA Tests with varied part geometry provide guidance for predicting the impact of part orientation changes on assembly rates and point to the importance of secondary interactions that can occur to increase the probability of parts interacting as when they slide down an assembly substrate. These measurements point to some complexity in the interactions that is not captured in the simple model. Future work will address the variation of assembly rates with part energy and test the ability of these methods to predict the effects of geometric scale on the process rate.

On the other hand, effects of $\varphi$ were measured to be within the experimental variation in the measurements. This is a necessary condition for an energy-based model of SA process kinetics and provides support for the energy-based model presented here. While there is some path dependency observed (Figure 23-a) the energy model provides substantial conceptual simplification and may still be very useful. Additional work is required to assess the adequacy 
of the energy ratio as an assembly parameter by evaluating assembly rates with varied energies across a range of part sizes eventually using multiple part sizes and bonding energies.

While these experiments are based on simplified systems that lack some effects seen in actual SA processes, results from this work provide unprecedented insight into the factors that affect part site interaction outcomes, and complement a process modeling efforts, such as the agent-based model described above (Massimo Mastrangeli et al., 2010). From an SA system design perspective, the reported discussion suggests that controlling directionality of part motion is not as important as controlling part orientation, part velocity (kinetic energy) and bonding energy. 


\section{CHAPTER 5:}

\section{MONTE CARLO STUDY OF SELF-ASSEMBLY DATA VARIATION}

SA tests presented in Chapter 4 contained substantial variation (as evidenced by the size of the error bars in Figure 23). It is hypothesized that such variation was largely due to the stochastic nature of part-site interactions. The present section tests this hypothesis through Monte-Carlo simulations of the experimental part-site interactions. Moreover, these simulations were used to evaluate a new testing strategy for minimizing the number of dropping tests required to get acceptable measurement accuracy. This strategy will be implemented in subsequent SA experiments.

\subsection{Introduction}

The pick-and-drop system showed significant inaccuracy in the landing location of the part drops of previous water-medium tests. The dropping process controls the location of the tool when dropping the part, but experiments showed that there is significant scatter in the landing locations, even when dropped from the same dropping location. The horizontal distance between a land location and its corresponding drop location served as a measure of dropping accuracy. In the water dropping tests, drop accuracy was measured with a standard deviation of approximately $6 \mathrm{~mm}$. This yielded a low certainty that the part would land on a desired site. Hence the procedure for water tests dropped parts at random locations uniformly over the entire assembly site area (Carballo \& Crane, 2014). The randomized drop locations provided every site equal probability to interact with a dropped part. Such condition allowed for Equation 10 (Chapter 4) to define the rate (or frequency) of part-site interactions as $r_{i}=$ 
$\left(n_{\text {sites }} \cdot A_{\text {site }} \cdot \sin (\varphi)\right) / A_{\text {total }}$, which is the area fraction of the total dropping area that corresponds to assembly sites. However, if there is an insufficient number of part drops, there would be a varying discrepancy between the actual $r_{i}$ value, and its approximation based on a uniform landing distribution. Therefore, any discrepancy and variation of $r_{i}$ propagates -as noise- to assembly rate measurements, as each part-site interaction contributes to such measurements (per Equation 5, assembly rate $\left.\dot{B}=\rho_{a} \cdot r_{i}\right)$. The present chapter evaluates the hypothesis that this source of error occurred in water-medium experiments (Carballo \& Crane, 2014) and could have been the primary source of the variation in the process measurements.

Monte Carlo simulations were used to estimate the impact of various sample sizes on the variation in the assembly rate data. The first section describes the simulation method. Then, the method is used to predict how many part-drops would be required to reach target accuracy levels. Accordingly, such conclusion provides an explanation for the large noise evidenced in watermedium experiments (Chapter 4). Moreover, this section concludes with an optimum dropping strategy to be implemented on future SA experiments. Monte Carlo simulation of the alternative dropping strategy provides estimates of how many part-drops should compose a single SA experiment, and how frequently assembly data should be acquired.

\subsection{Monte Carlo Simulation Procedure}

A Monte Carlo simulation is an approach for making predictions about the behavior of a physical or mathematical model for which one or more variable values can vary based on a known probability distribution. First, random values are generated for input variable(s), following a known (or assumed) probability distribution. Each random value is input into the physical or mathematical model of interest to calculate the resulting outcome. The step of generating random input and calculating the outcome is commonly called a "simulation trial". 
By repeating the calculation for a large number of simulation trials, an accurate estimate of the distribution of outcomes can be developed (Bouleau \& Lépingle, 1994; Maybeck, 1979). The output values can be analyzed for different purposes. For instance, one can use the Monte Carlo approach to analyze the statistical uncertainty of a system; analyze the sensitivity of the system to a certain parameter; and/or simulate complex dynamical systems; among other applications.

The present Monte Carlo study assesses the effects that the statistical uncertainty of the dropping locations imposes on SA experiments. More specifically, the effects are quantified by the variation of rate of interactions. In order to predict the rate at which parts and sites interacted, part-drops were simulated and each simulated drop was assessed as to whether the landing occurred inside the boundaries of an assembly site. Every on-site landing produces one part-site interaction. If the probability of assembly on contact, $\rho_{a}=1$ is assumed, then every partsite interaction would generate an assembly. This condition is representative of lower kinetic energy assembly conditions as analyzed in Chapter 4. Simulations monitored the number of assemblies after a finite increment of part-drops; thus allowed for calculating the rate of part-site interactions as the number of part drops increased. The following text describes how landing locations were simulated. Subsequent sections explain how the simulation output was analyzed to extract rate predictions.

\subsubsection{Method for Simulating Landing Locations}

The pick-and-drop system drops parts with a certain degree of accuracy. In other words, parts do not land exactly below the dropping location. Monte Carlo simulations were based on a set of equations that modeled the drop locations, and resultant part landing locations. First, a set of dropping locations was generated to approximate a uniform spatial distribution over the defined area. Using Matlab 2013b, the function 'rand' generated uniformly distributed random 
numbers between 0 and 1 . The following equation -written as a Matlab command line- depicts the model for simulating a drop location.

$$
\mathrm{D}=\left[\left(\operatorname{rand} \cdot \operatorname{size}_{\mathrm{x}}\right),\left(\operatorname{rand} \cdot \operatorname{size}_{\mathrm{y}}\right)\right]
$$

Equation 11

The coordinate point $\mathrm{D}$ is a 2-element vector, defining the $\mathrm{x}$-y coordinates of a single drop-location. The scalars $\operatorname{size}_{\mathrm{x}}$ and size $\mathrm{y}_{\mathrm{y}}$ are the dimensions of the rectangular drop-area, within which all drop locations are uniformly distributed. Next, a Gaussian probability distribution was used to model the distribution of part landing locations relative to the target (drop) position. The landing location was defined as follows (also written as a Matlab command line).

$$
L=D+[(\operatorname{randn} \cdot \sigma),(\operatorname{randn} \cdot \sigma)]
$$

Equation 12

The last term of the right side of Equation 12 represents the inaccuracy of the pick-anddrop system. The Matlab function "randn" generates random numbers, following a Gaussian probability distribution, with mean $=0$, and variance $=1$. The scalar $\sigma$ is the standard deviation of the previously measured distance between landing locations and drop location. This value is a direct measure of the drop-process inaccuracy (e.g. $\sigma=0$ for a perfectly accurate system). The $\sigma$ value was previously measured for different test conditions (water medium, and air medium with different drop heights). A procedure for measuring $\sigma$ is explained below.

\subsubsection{Method for Measuring Inaccuracy of Pick-and-Drop System}

The standard deviation $\sigma$ was acquired from measuring the inaccuracy of the pick-anddrop system. First, the drop location (D) was programmed into the pick-and-drop system. A reference point was drawn on the landing surface, and defined as the origin of the coordinate system defining the drop and landing locations. A minimum of 50 parts were dropped (this number provided a consistent average measurement). Top-view digital images captured the position of each landing location (see Figure 27). The imaging equipment included an IDS 
camera (UI-1460SE) with a 3X compact telecentric lens (Edmund Optics). Images were postprocessed in order to maximize the grayscale contrast between target points and the white background. Photoshop was used to measure the distance between the reference point and the dropped part (i.e. landing location L).

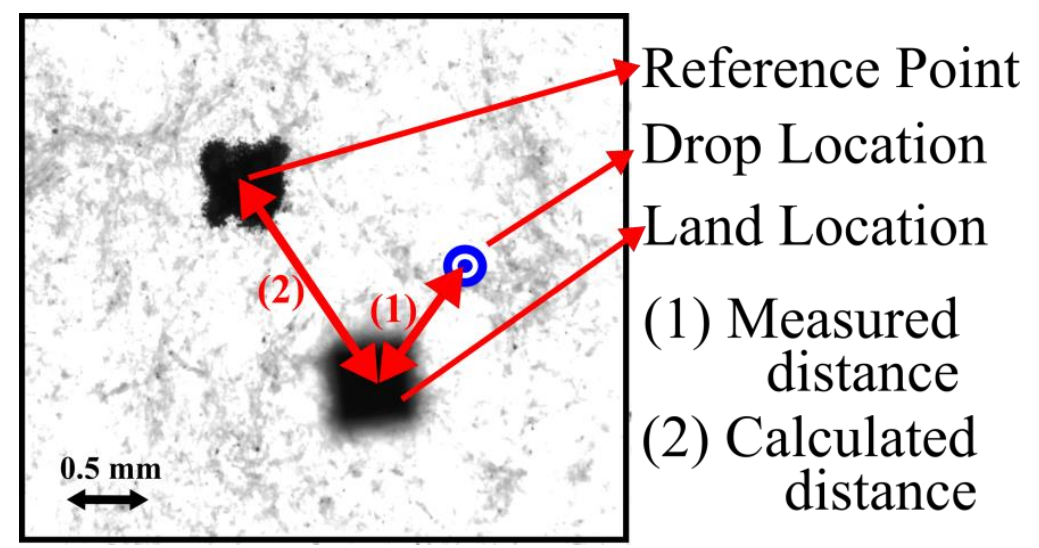

Figure 27 Sample image captured for measuring drop accuracy of the pick-drop system. The drop location (not drawn to scale, for clarity purposes) was located $0.1 \mathrm{~mm} \times 0.1 \mathrm{~mm}$ away from the reference point. The land location was acquired as the centroid of the part. The part is displayed out-of-focus due to the difference in height between the reference point and the top surface of the part.

The measured distance allowed for calculating the distance between the drop location, and the landed part. Measurements were repeated for water medium, and for two different drop heights in air-medium (representing a low end and high end of the range to be tested in future SA experiments). For each configuration, a total of 50 part drops provided average and standard deviation values. Air-medium measurements required a coating of the landing surface, in order to absorb impact, and prevent parts from bouncing off the actual landing location. A thin, transparent glycerol coating achieved such purpose, while allowing the top-view image to capture both part and the reference point (underneath the coating). Results of standard deviations of the calculated distances between drop and landing locations are depicted in Table 4. The standard deviation of the calculated distances was then inserted as $\sigma$ into the command 
line in Equation 12, which was used by the Monte Carlo simulations to generate landing locations.

Table 4 Summary of drop accuracy measurements

\begin{tabular}{|l|c|}
\hline & $\begin{array}{l}\text { Standard deviation of } \\
\text { measured distance }(\sigma)\end{array}$ \\
\hline Water medium (capillary gripper) & $6 \mathrm{~mm}$ \\
\hline Air medium (Vacuum gripper), parts dropped from 3 mm height & $0.25 \mathrm{~mm}$ \\
\hline Air medium (Vacuum gripper), parts dropped from $10 \mathrm{~mm}$ height & $0.29 \mathrm{~mm}$ \\
\hline
\end{tabular}

\subsubsection{General Procedure for Monte Carlo Simulations}

Sampling strategy is defined by 1) the number of part-drops before each time assembly data was sampled; and 2) the total amount of data acquired for each SA test. Monte Carlo simulations evaluated whether the sampling strategy of water-medium tests was a contributor to scatter in the SA test data. First, simulations replicated the drop area size and sampling strategy implemented in water-medium experiments (Chapter 4). However, the area surrounding a single strip (with 20 assembly sites) was considered instead of evaluating an area containing 12 strips (as implemented in water-medium tests). The right/left boundaries depict the edges of the strip, past which there are no more assembly sites. Accordingly, the upper/lower boundaries of the simulation drop area (red outline in Figure 28) corresponded to adjacent strips. Periodic boundary conditions for the upper/lower boundaries simulated parts landing on adjacent strips. A detailed procedure for applying such boundary conditions is described below.

Equation 11 generated a random drop-locations, with size $\mathrm{x}_{\mathrm{x}}$ and size $_{\mathrm{y}}$ equal to the width and height of the drop area (delimited by red outline in Figure 28), respectively. The origin of the coordinate system was the center-left corner of the drop area. Next, Equation 12 calculated the landing location yielded by the corresponding drop. In this case, $\sigma=6 \mathrm{~mm}$ represented the 
accuracy of the water-medium pick-drop system. This was repeated for the target number of drops.

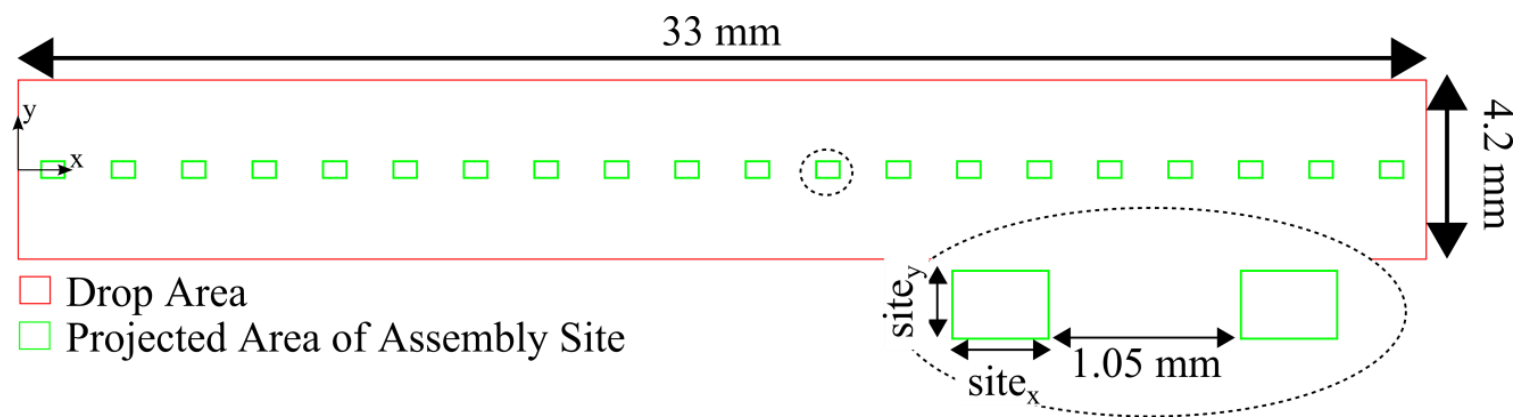

Figure 28 Illustration of boundaries for drop area and assembly sites. Drop locations were generated within the drop area. Assembly sites are $0.6 \times 0.6 \mathrm{~mm}^{2}$, however they were inclined to a certain angle (angle of incidence, $\varphi$ ), which made the projected area smaller along one direction. This geometry reflects the same implemented for water-medium experiments, and $\varphi=$ $45^{\circ}$.

A single test simulation ended after the last part-drop simulation, and percentage of landing locations occurring inside of an assembly site (delimited by green boundaries in Figure 28) was calculated. The whole simulation process (generate random drops, calculate landings, and calculate on-site landings fraction) was repeated for 12 strips, as this was the number of strips utilized for water-medium tests. The final result of one simulation trial accounted for the sum of part-drops, and on-site landings from each strip.

Periodic boundary conditions were applied for the edges adjacent to another strip. These conditions consider parts that were dropped on top of one strip, but land on adjacent strips (or the area surrounding them) due to the difference between dropping and landing location. Hence, the landing locations that occurred past such boundaries were considered as if landing inside the drop area. For instance, a part that exited the bottom boundary would actually fall on another strip below, and have a distance $y_{1}$ from the top of such strip. For the simulation, this was modeled by placing the part at a distance y1 from the top of the top boundary of the current strip. 


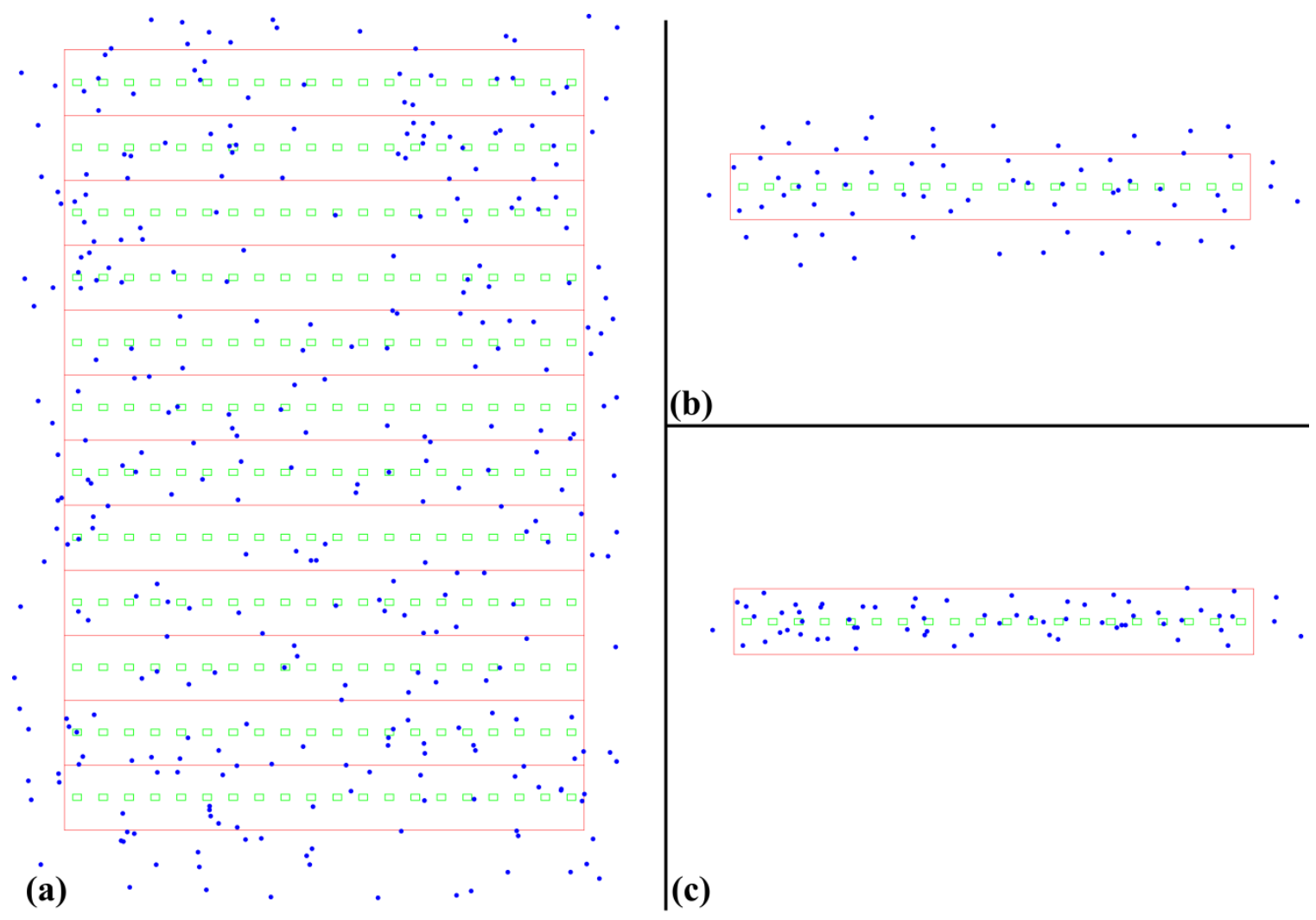

Drop Area $\square$ Projected Area of Assembly Site Landing Locations Figure 29 Configurations of assembly site geometries, as implemented in Monte Carlo simulations of water-medium experiments. (a) Schematic of 12 strip configuration, as implemented in water-medium tests. (b) Schematic of single strip configuration. Landing locations lying beyond the upper and lower edges were considered outliers. (c) Periodic boundary conditions were applied to outliers beyond the upper and lower edges. These outliers were considered as if landing inside the drop area. These conditions simulate outliers landing on adjacent strips.

Figure 29(a) allows visualizing the array configuration of 12 strips. Accordingly, strips 211 had periodic boundary conditions on the upper/lower boundaries of the drop area. For strips 1 and 12, boundary conditions were only applied to the upper edge (strip 1), or lower edge (strip 12). Figure 29 (b) illustrates the case with part landings outside the drop area (i.e. without applying periodic boundary conditions). Figure 29 (c) illustrates the opposite case where the same outliers are considered inside (i.e. with periodic boundary conditions applied). 


\subsection{Monte Carlo Simulations of Part-Site Interactions}

After simulating a desired number of drops over 12 strips, the percentage of on-site landings (where each on-site landing produces one part-site interaction) was calculated as the ratio of total number of on-site landings over the total number of assembly sites (240 sites corresponding to 12 strips with 20 sites each). Such result corresponded to that of 1 test simulation. In order to assess the uncertainty in a single test, the test simulation was repeated one thousand times. Average and standard deviation of the percentage of parts landing in assembly locations were calculated for these simulated tests. Furthermore, the ratio of standard deviation over the average defined the "relative standard deviation" (R.S.D.). Figure 30 illustrates how the average and R.S.D. changed as the part-drops were increased. The left axis is the scale for the average magnitude, while the right axis shows the R.S.D. values.

For the water-medium experiments, Figure 30 illustrates that a total of 12,000 parts (uniformly dropped over 12 strips) were needed to reduce variation below 5\% R.S.D (which could be considered a reasonable, initial target). Each water-medium SA experiments involved a total of about 1,000 parts, dropped over 12 strips. Therefore, the sampling strategy of watermedium experiments possessed a variation of on-site landings of about $18 \%$ R.S.D., according to Figure 30. Consequently, variation of on-site landings would naturally induce noise to the assembly data. However, comparison of Monte Carlo simulations with experimental data requires calculating assembly rate.

The present section has presented the basic procedure for simulating part-site interactions, and predicting how many parts land on sites with a given dropping condition, and sampling strategy. Next, the simulation algorithm was further expanded to post process simulation results and output assembly rate predictions. The next section depicts the simulation 
algorithm for calculating assembly rate, and also presents simulations that estimate such sampling variation effects on experimental data.

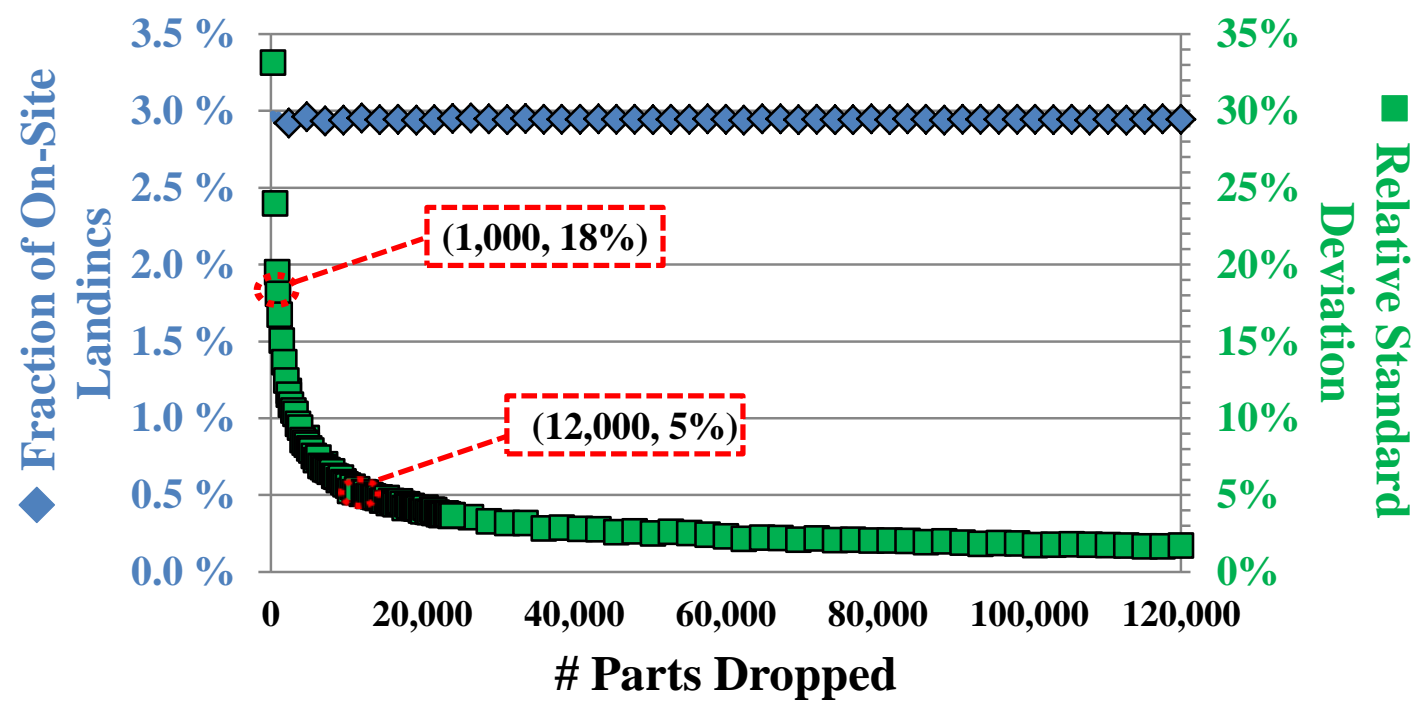

Figure 30 Progress of on-site landings for incremented number of parts dropped. The variation of on-site landings is measured by the relative standard deviation (right axis). The vertical line depicts the sampling amount used for previous water-medium tests (Chapter 4); this was equal to approximately 80 parts.

\subsection{Monte Carlo Simulations of Assembly Rate}

Assumptions of probability can be combined with the on-site landing predictions in order to generate estimates of assembly counts. For example, assembly probability, $\rho_{a}=1$ assumes that every on-site landing generates an assembly. A set of simulations was based on $100 \%$ assembly probability and the geometry depicted in Figure 29(c). When an on-site landing occurred, the corresponding site was registered as "filled" (i.e. an assembly), so that subsequent landings on that site would not generate additional assemblies. Simulations extracted the number of assemblies produced from incremental numbers of drops. Figure 31 displays the results of several simulations, portraying how assembly progressed with incremented part-drops. 
The plots shown for each simulation demonstrate the $1^{\text {st }}$ order reaction nature of the stochastic assembly process. Each simulated data set was fitted to the integrated rate law equation, of $1^{\text {st }}$ order reaction:

$$
\frac{B(n)}{A_{\mathrm{o}}}=1-e^{-k_{B} \cdot n}
$$

Similarly to Chapter 4 definitions, $B(n) / A_{o}$ is the fraction of assembly sites filled with a part-site assembly; $k_{b}$ is the reaction rate constant for (correct) assembly formation, and $n$ is the number of parts dropped. Unlike the competing reactions seen in Chapter 4, the present simulations neglect the formation of incorrect assemblies (in other words, assume that orientation probability $\rho_{o}=1$ ). Considering the formation of incorrect assemblies would not significantly alter the conclusion from the present simulations.

Simulations results were interpreted in terms of initial assembly rate. The assembly rate, $\dot{B}$ was defined as the derivative of the function of parts-dropped, $B(n)$. Accordingly, the derivative of Equation 13 becomes

$$
\frac{d}{d n}\left(\frac{B}{A_{0}}\right)=k_{B} \cdot e^{-k_{B} \cdot n}
$$

Hence, the rate of change of $\dot{B} / A_{o}$ becomes $k_{B}$ as $\mathrm{n} \rightarrow 0$. For this reason, the rate constant can also be considered as the initial rate of change. The reported "assembly rate" results from all the Monte Carlo simulations in this chapter refer to initial assembly rate (i.e. $k_{b}$ ).

The simulations of Figure 31 illustrate the difference between best fits. Such difference arises from variation of on-site landings (which was illustrated in Figure 30) on a finite number of sites. As the number of assembly sites increases, the answer should converge to a consistent average and a lower standard deviation of the assembly (i.e. rate constant $k_{b}$ ). A low standard deviation is desired; because, it is a direct measure of the data scatter caused by the stochastic nature of SA interactions. 


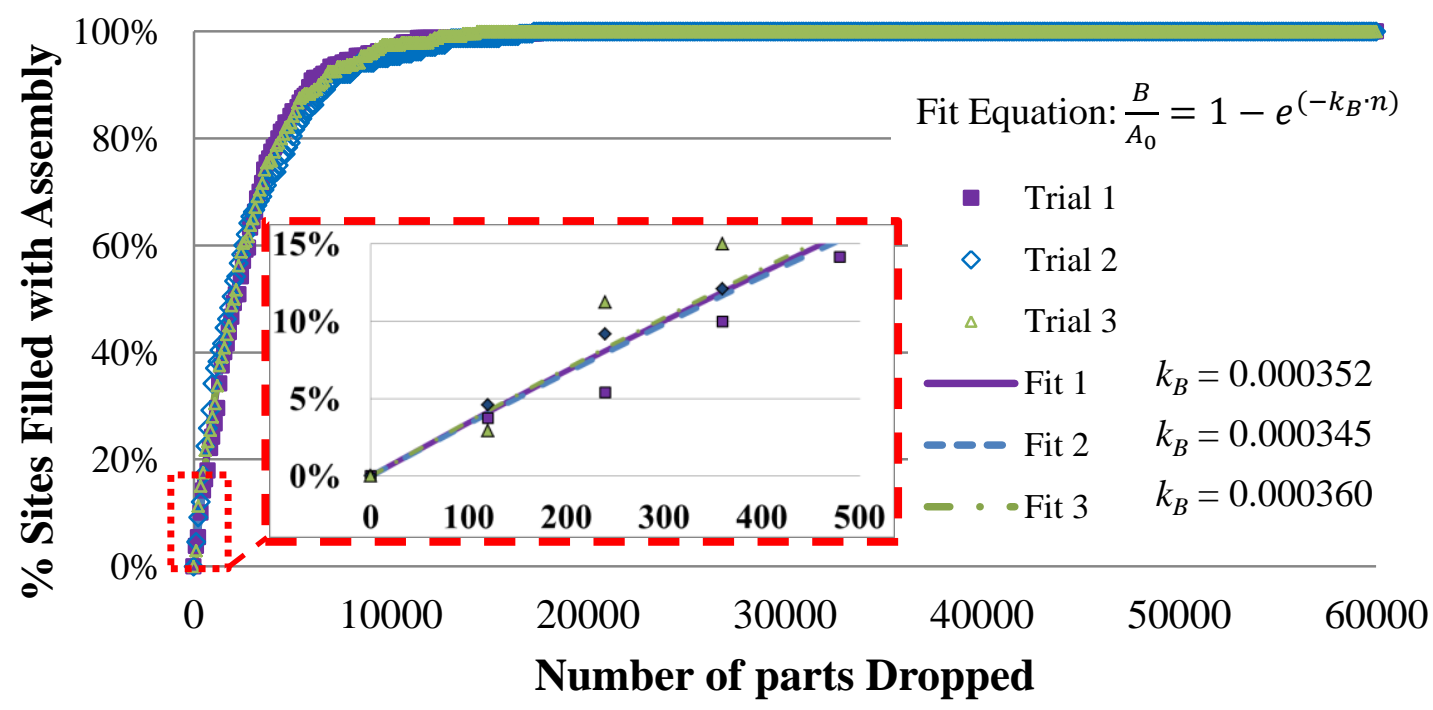

Figure 31 Results from 3 simulation trials, displaying the change in assembled sites $\left(B / A_{o}\right)$ with the increasing number of parts dropped. Simulations are based on the assumption that any onsite landing produces an assembly (i.e. $\rho_{a}=1$ ). Solid lines represent regression fits of each simulation trial to Equation 13. The rate constant values are given for each corresponding best fit.

The assembly rate results from water medium experiments (Chapter 4) showed error bars of significant size (refer back to Figure 23, in Chapter 4). The corresponding R.S.D. values lied between $5 \%$ and $30 \%$, and were derived from up to 5 data points of each test configuration. Each of those tests performed up to 1,000 drops over 12 strips. The R.S.D. values seen in watermedium tests were compared with Monte Carlo simulations. The simulation conditions used for Figure 31 trials were implemented for a set of 1,000 simulation trials with up to 1,000 parts dropped in each trial. One thousand simulation trials allowed calculation of meaningful statistics about the distribution of the measurements. Additional sets of 1,000 simulation trial were repeated for different amounts of parts dropped per simulation. Each simulation set extracted the average assembly rate constant, $k_{b}$. 


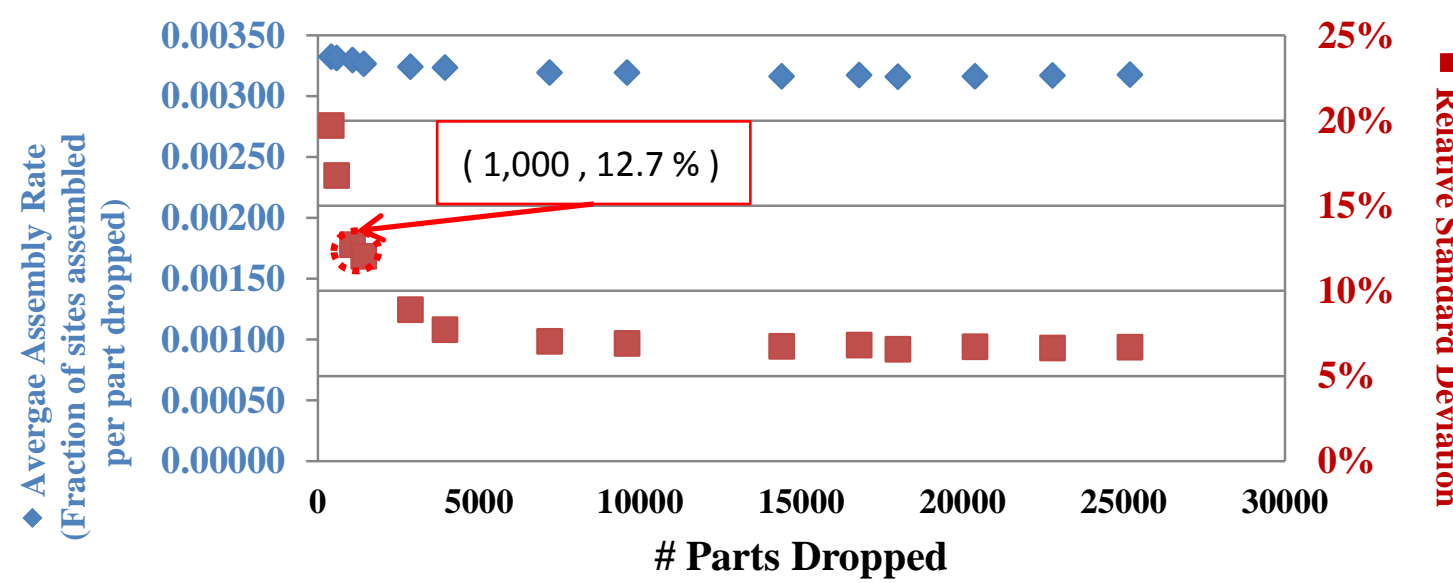

Figure 32 Results from Monte Carlo simulations considering water-medium test conditions. Plot depicts progress of average assembly rate as the number of parts dropped was increased.

Figure 32 allows for tracking the change in average assembly rate (left y-axis), and its corresponding R.S.D. value (right $\mathrm{y}$-axis) as the number of parts increases. The $\mathrm{x}$-axis displays the number of parts dropped. R.S.D. decreases non-linearly with respect to the number of partdrops.

Additionally, Table 5 compares the variation (in terms of R.S.D.) of $k_{b}$ values between water-medium tests and simulated trials, both involving 1,000 part drops. Simulation results presented a comparable variation to that of SA tests. Since the simulations assume that $\rho_{a}=1$, the specific assembly rate is not expected to match, but, these results do provide useful estimates of the expected experimental variation. The simulation involved 1,000 trials, while SA tests only involved up to 5 data points the accuracy of the R.S.D. values is limited. However, the simulation shows that large errors compared to those seen in most of the self-assembly tests could be generated by the stochastic variations in the test system. 
Table 5 Comparison of assembly rate variation between water-medium experiments and Monte Carlo simulations

\begin{tabular}{|l|l|l|}
\hline & Water-Medium SA Tests (Chapter 4) & Simulation Results \\
\hline R.S.D. (\%) & Between 5\% and 30\%* & $12.7 \%$ \\
\hline
\end{tabular}

* These values were extracted from the error bar magnitudes, presented in water-medium experiments results (see Figure 23, Chapter 4).

Figure 33 displays the distribution of assembly rate values produced by the same set of simulation trials that dropped 1,000 parts. The distribution adequately fits a Gaussian distribution with an R.S.D. of $12.7 \%$. Furthermore, the same plot also displays the distribution of the same assembly rate values, when sets of three simulations are averaged. The narrower distribution represents the probability for any set of 3 experiments to yield the corresponded rate value as an average. This information allows understanding how much are the averages expected to vary from a low number of trials such as the tests reported in Chapter 4.

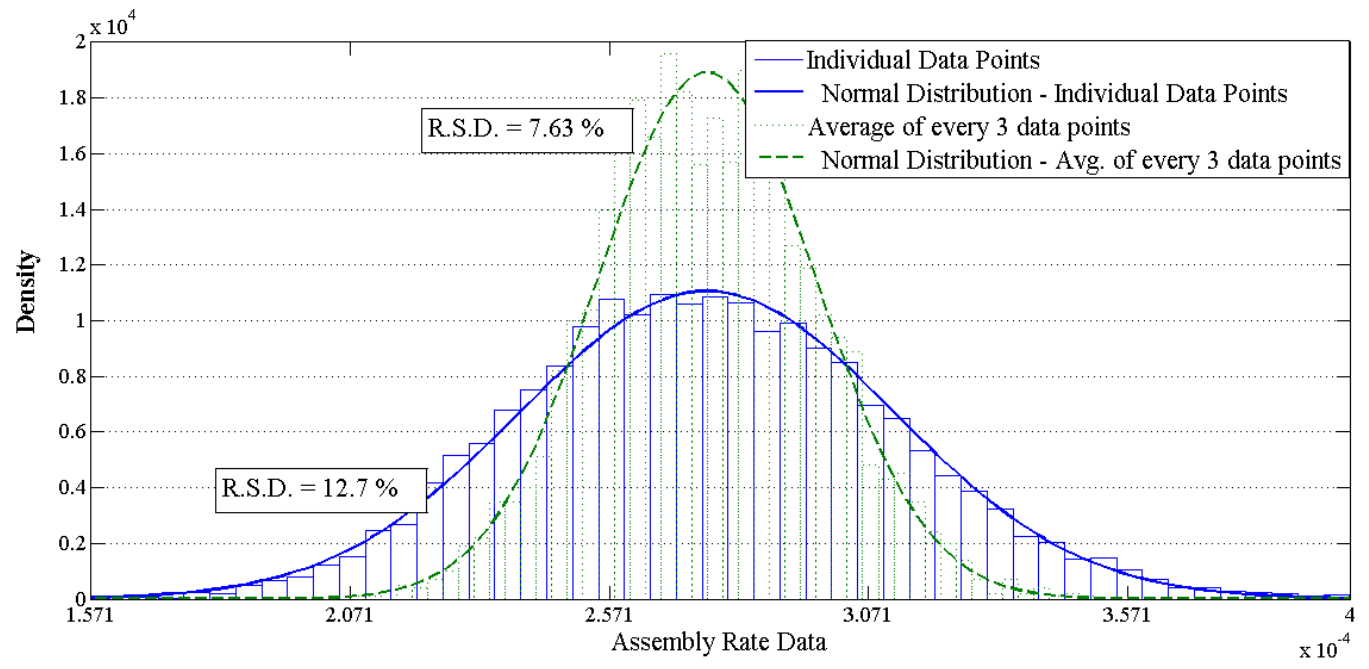

Figure 33 Histogram plots of assembly rate data from Monte Carlo simulations replicating watermedium testing conditions. The solid-line represents the distribution of individual data points. The green line represents the average of groups of 3 data points.

A large number of simulation trials, and part drops is desired for minimizing experimental variation, but it is time consuming to run longer tests and analyze the data. Additionally, when a large number of parts are dropped over a single assembly region, the parts 
are more likely to interact with other parts that are already assembled yielding less incremental information with additional dropped parts. Consequently, an optimum test strategy is required to minimize variation, while avoiding parts landing on existing assemblies. The following section presents an analysis of reaction kinetics for defining a sampling limit for avoiding landings on existing assemblies. Moreover, next section also includes a Monte-Carlo study for evaluating an optimum test strategy.

\subsection{Analysis of Optimum Strategy for SA Tests}

\subsubsection{Linear Approximation of $1^{\text {st }}$ Order Kinetics Reaction}

When a part lands on an unavailable assembly site, no assembly can occur. Therefore, as the assembly sites fill up, there is less value in dropping additional parts. This is seen in Figure 31 as the slope of the curves decreases. To obtain the maximum amount of information from each part drop, it is preferred to remain in the linear regime of the assembly process. Each simulation trial shall be kept at a low fraction of assembled sites. More specifically, the $B(n) / A_{o}$ curves (as plotted in Figure 31) should be kept within the linear regime. This minimizes the number of parts landing on existing assemblies and thus maximizes the information obtained from each part drop. Moreover, the linear regime allows for a simpler computation of assembly rate, thus making the simulations less computationally expensive. The present section derives criteria for systematically selecting the linear range of the $B(n) / A_{o}$ curve of each simulation trial. It also explains a less expensive approach for calculating assembly rate.

As discussed above (when deriving Equation 14 for calculating assembly rate), the beginning portion of the integrated rate law equation of $1^{\text {st }}$ order can be approximated to a line with slope equal to $k_{B}$. The accuracy of such approximation decreases as more part drops occur 
(i.e. as $n$ increases). Figure 34 illustrates how the line $B=k_{B} \cdot n$ approximates Equation 13 . The error depicted in Figure 34 represents the error in predicted assembly state and is defined as

$$
\text { Linear Approximation Error }=\frac{\left|B(n) / n-k_{B}\right|}{k_{B}}
$$

Equation 15

where $B(n)$ is defined by Equation 13. The error magnitudes and corresponding values of $B(n)$ are true for any $k_{B}$ value. Only the scale of the horizontal axis $(n)$ would be specific to $k_{B}$. Thus, Monte Carlo simulations can output $k_{B}$ values through this line approximation.

As illustrated by Figure 34, a linear approximation with less than 5\% error requires that the assembled parts are less than $9.83 \%$ of the sites. Monte Carlo simulations confirmed this theory. A set of 1,000 simulations were performed with the same geometry conditions as described above: 12 strips, with the same drop area, and strip geometry implemented for watermedium tests (Figure 28). A total number of parts were dropped over all strips, while keeping the assembled-sites fraction $\left(B / A_{o}\right)$ below $9.83 \%$. Part-drops were generated in 10 uniform increments.

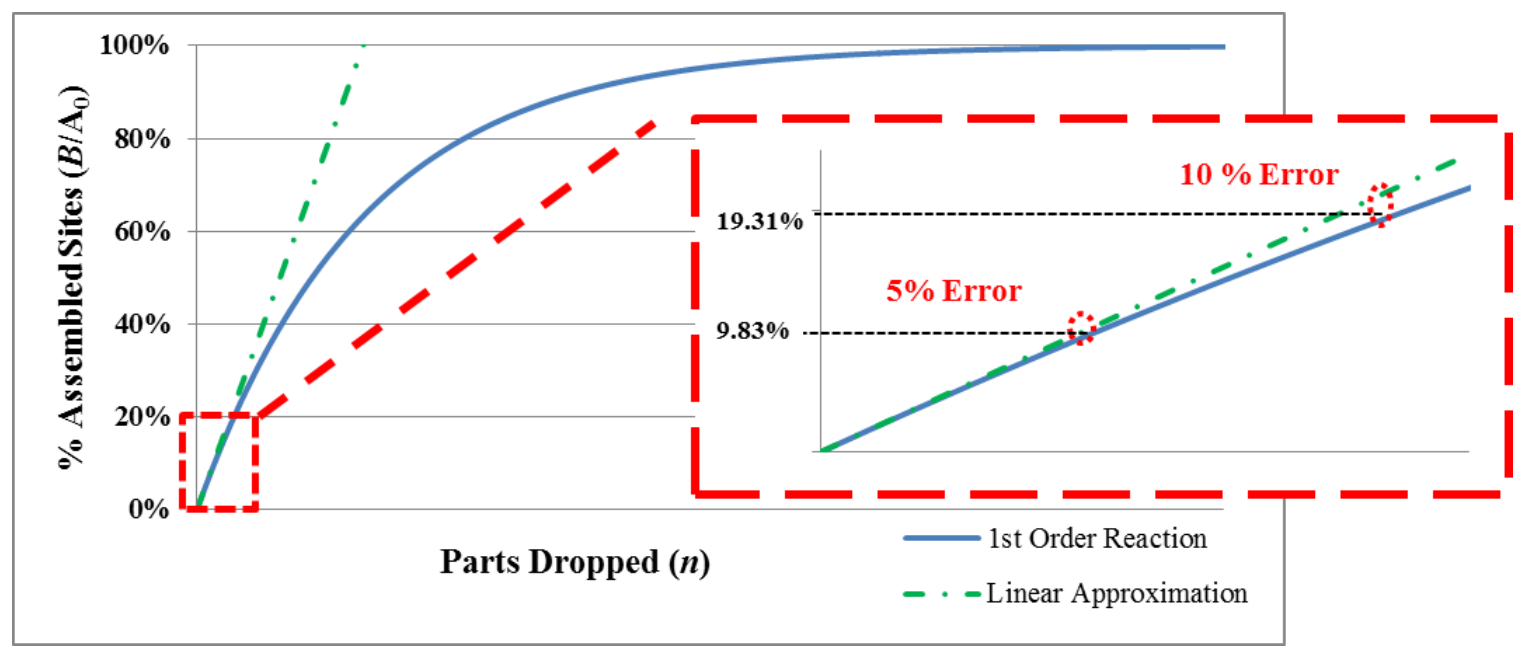

Figure 34 General curve of the integrated law of a $1^{\text {st }}$ order reaction. The line $B=k_{b} \cdot n$ approximates the beginning portion of the $1^{\text {st }}$ order reaction curve. The zoomed-in view illustrates the position where error amounts to 5\% and $10 \%$ (chosen as representative values for understanding the accuracy of this approximation). When assemblies amount to less than $9.83 \%$ of all assembly sites, the linear approximation is considered adequate. 
Two different analysis methods extracted assembly rate from the same simulation results. For the first method (regression fitting method), the fraction of sites filled (i.e. $\left.B(n) / A_{o}\right)$ was acquired after each drop increment (and for of the 1,000 simulations). Next, each of the simulation data sets $\left(B(n) / A_{o}\right.$ vs. n) was regression-fitted to the first order reaction (Equation 13), to extract $k_{B}$ from each fit. The second method was to divide the total assembly fraction by the total number of part-drops. Such ratio signified the slope of a line, as drawn by Figure 35. Following Equation 14 for $B(n) / A_{o}<9.83 \%$, this slope corresponds to an approximation of the rate constant value. Figure 35 depicts the results from the present simulation, and the error between both analysis methods.

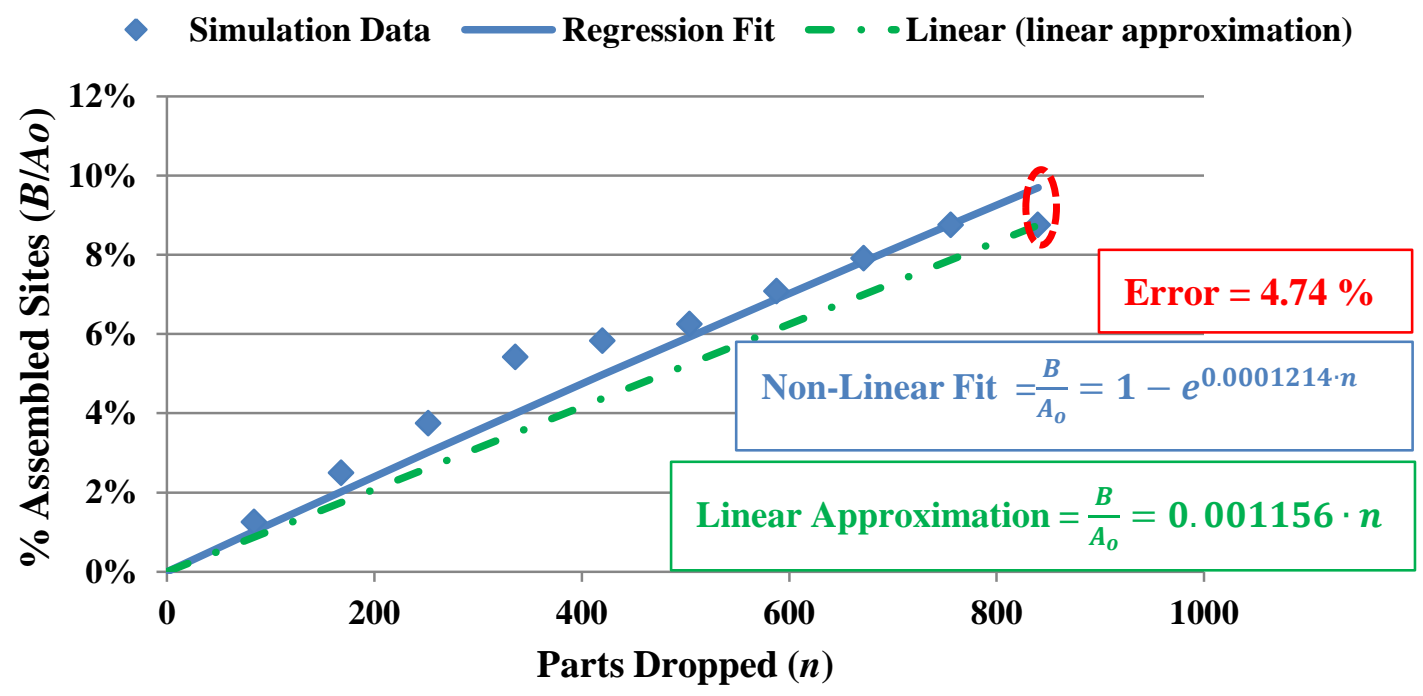

Figure 35 Comparison between regression fitting, and linear approximation for acquiring assembly rate data from Monte Carlo simulations. Drop area is that depicted in Figure 28, and a total of 840 parts were dropped (in 10 uniform increments) over 12 strips. Standard deviation, $\sigma$ $=6 \mathrm{~mm}$, simulating the dropping system for water-medium experiments.

The limit imposed by the linear approximation (keeping assembled sites below 9.83\%) defines a maximum number of part drops before acquiring assembly data (or in other words, a minimum sampling frequency). On the other hand, Section 3.4 presented an argument for 
increasing the number of parts-drops in order to reduce the inherent variation from low-sampling effects. Therefore, our sampling strategy must achieve a balance between these two constraints.

\subsubsection{Revised Strategy for Assembly Rate Measurements}

Assembly measurements for sampling $B(n)$ must comply with two requirements:1) Measurements must be performed before reaching $B(n) / A_{0}=9.83 \%$ so that data can be fitted to a line; and 2) number of samples must be high enough to reduce the effects of on-site landing variations by improving the accuracy of the uniform probability assumption. According to simulations from Section 0, when more than 20,000 part drops are dropped the relative standard deviation (of assembly rate) drops just below 7\%. On the other hand, dropping this large number of parts on a single strip would certainly generate assemblies on $>99 \%$ of the sites (as evidenced in simulations from Section 0) and have a high variation due to the small number of sites available. Therefore, a strategy for creating part-site interaction needs to comply with both requirements, and while measuring enough interactions to get a good estimate of the actual assembly probability.

For water-medium experiments, part-site interactions were created on a large array of assembly sites (12 strips with about 24 sites each). Parts were dropped uniformly over the entire region. Many of the parts were dropped over the regions between strips with no chance of assembly. This increases the number of parts that must be dropped in order to get sufficient partsite interactions for a good measurement of the assembly probability. The water-tests were done at low velocity for maximum assembly probability. Testing in air at higher kinetic energies will have a lower assembly probability — further increasing the number of parts that need to be dropped in order to get an accurate measurement of the assembly probability. A revised 
approach is required that increases the efficiency of the process by only dropping parts in the vicinities of the actual assembly locations.

Instead of assembling numerous strips with assembly sites simultaneously, the new strategy targeted individual strips in a serial fashion. Parts were dropped on a single strip at a time until it approached the limit of the linear assemblies. In the new approach, the assemblies from each strip are measured, and accumulated (results from the current strip were added to the results of previous strips). This approach allowed creating as many assemblies as required to reduce intrinsic variation, while not filling more than $9.4 \%$ of each strip (and of all assembly sites, accordingly). Moreover, slight changes on the geometry of assembly sites and drop area increased the probability of on-site landings (and thus the signal-to-noise ratio).

First, assembly sites were packed together more closely. While the assembly site area remained constant, the horizontal spacing between sites was decreased to $0.72 \mathrm{~mm}$. This reduced-spacing allowed for fitting more sites over the same length of a strip ( 25 sites instead of 20). Secondly, the shorter dimension of the drop area was further decreased. This change allowed a more focused part dropping with spaces between strips in which no parts were dropped, targeting a higher on-site landing percentage. The modified dimension was evaluated through Monte Carlo simulations. The following section presents a simulation based analysis of the effects from reducing the drop area.

\subsubsection{Analysis of Results}

Monte Carlo simulations were performed to evaluate our new test strategy, and devise an optimum drop area size. The goal was to measure variation of assembly rate as a function of number of parts dropped, and drop area geometry. A number of parts were dropped on an increasing number of strips. The number of drops per strip was adjusted to fill each strip below 
$9.8 \%$ of its sites. A resulting average of $9.6 \pm 0.03 \%$ (in each simulation set) of assembled sites ensured that this condition $(<9.8 \%)$ was met. Each part drop was evaluated whether it landed inside the boundary of an assembly site (which is considered an on-site landing). Similarly to previous simulations, it was assumed that any on-site landing generated an assembly (i.e. $\rho_{a}=1$ ). However, these new simulations only consider one strip at a time, thus eliminating the need for periodic boundary conditions. Results were analyzed after regular increments in number of strips. Assembly data was evaluated as the fraction of the total number of sites; i.e. $B / A_{0}$. Initial assembly rate (as defined by Equation 14 when $n=0$ ) equaled the total fraction of assemblies by the total number of parts dropped. For every incremental number of strips, 1,000 simulation trials allowed extracting an average and standard deviation of assembly rate.

A $1^{\text {st }}$ set of simulation considered the geometry and conditions of water-medium experiments. These conditions include a drop area as large as the uniform fraction surrounding each individual strip (see Table 6). Results and geometry are depicted in Figure 36. However, the strips were still addressed in a serial fashion (instead of 12 at a time as before). The purpose of this set of simulations was to compare the original test strategy (testing 12 adjacent strips simultaneously) with the new strategy (which tests 1 strip at a time). Figure 36 displays the results from these simulations, in terms of average assembly rate, and its corresponding R.S.D. value. This plot allows visualizing the minimum number of drops required for reducing R.S.D. to a specific level.

Table 6 Parameters for simulations considering water-medium experiment conditions, and dropping over 1 strip at a time. Results from these simulations are presented in Figure 36.

\begin{tabular}{|c|c|}
\hline$\sigma(\mathrm{mm})$ & $6 \mathrm{~mm}$ \\
\hline Drop Area $\left(\mathrm{mm}^{2}\right)$ & $33 \times 4.2$ \\
\hline Assembly Site Area $\left(\mathrm{mm}^{2}\right)$ & $0.6 \times 0.954$ (corresponding to c $=3)$ \\
\hline Inter-site spacing $(\mathrm{mm})$ & 1.05 \\
\hline Number sites per strip & 20 \\
\hline Number of part-drops per strip & 10 \\
\hline
\end{tabular}




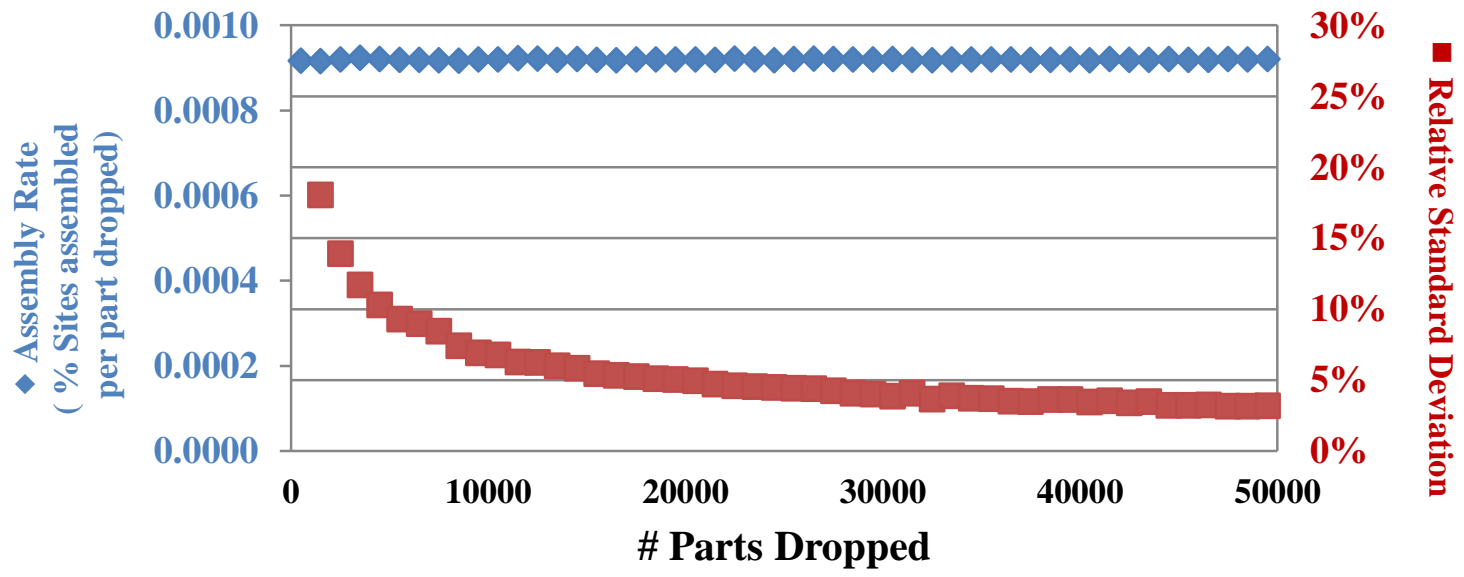

(a)

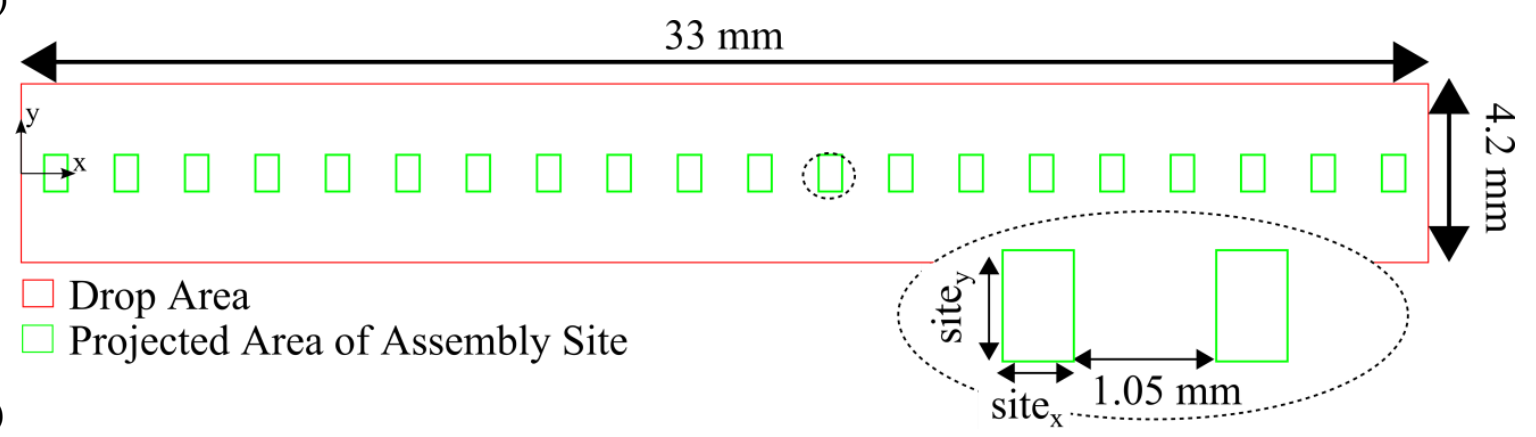

Figure 36 (a) Results from Monte Carlo simulations considering water-medium test conditions ( $\sigma$ $=6 \mathrm{~mm}$ ), and revised strategy (single strip instead of 12 strips). Plot depicts progress of average assembly rate as the number of parts dropped increased. Simulations evaluated a single empty strip after every 15 parts dropped. Each data point corresponds to an average of 1,000 trials.(b) Geometry of drop area and assembly sites implemented for simulations in (a).

According to Figure 36, simulations estimate requiring about 20,000 parts for reducing assembly-rate R.S.D. to 5\%. On the other hand, previous simulations that followed the original test strategy (testing 12 strips simultaneously) required 25,000 parts for dropping the R.S.D. just below $7 \%$. Hence, under these conditions, the new strategy does not show a significant improvement. Such large number of parts still signifies a time-consuming experiment procedure. Much of the lack in efficiency is likely due to parts that land outside the test area in this "single strip" configuration. These samples cannot assemble in the single strip configuration whereas in the prior system, they might have assembled on a neighboring strip. Such was confirmed by 
repeating the same set of simulations (also implementing the new single-strip strategy) but applying the periodic boundary conditions to the upper/lower edges of each individual strip. Such conditions allowed understanding the effects from having multiple, adjacent strips simultaneously. These additional simulations showed that less number of part drops (i.e. 5,200) was needed for achieving an assemble rate R.S.D. $=5 \%$. Hence, assemblies significantly increase when having adjacent strips to receive parts where there is a large uncertainty in their dropped position.

The new strategy offers a way to test as many drops as needed (for reducing variation) without filling the assembly sites. However, applying this revised strategy is time consuming when the drop-system is inaccurate, thus having a large standard deviation for landing locations (such as $\sigma=6$ ). However, there is much room for improvement as the drop area could be further decreased, so fewer parts would be needed to extract useful statistics. The following set of simulations will consider smaller drop areas, and conditions for future tests. Moreover, future tests involve air-medium, for which its drop system offers a much better drop-accuracy $(\sigma=0.25$ $\mathrm{mm})$. Therefore, this also reduced the concerns about requiring a large number of parts.

A new set of simulations considered the accuracy of air-medium experiments $(\sigma=0.25$ $\mathrm{mm})$. Every set simulated a gradually smaller drop-area, thus focusing drops on the sites and increasing the percentage of on-site landings. Geometry of drop areas and strips, for each simulation, are illustrated in Figure 37. The first, larger, drop area was the same implemented for a single strip in water-medium tests $(33 \mathrm{~mm} \times 4.2 \mathrm{~mm})$. The second and third drop areas where decreased by half of the previous one (33 $\mathrm{mm}$ x $2 \mathrm{~mm}$, and $33 \mathrm{~mm}$ x $1 \mathrm{~mm}$, respectively). The third and smallest drop area only covers the width of assembly sites, and the length of the strip. Moreover, the closely packed geometry of assembly sites was considered, as it will also be 
implemented on future tests (i.e. inter site spacing $=0.72 \mathrm{~mm}$, fitting 25 sites along the $33 \mathrm{~mm}$ long strip).

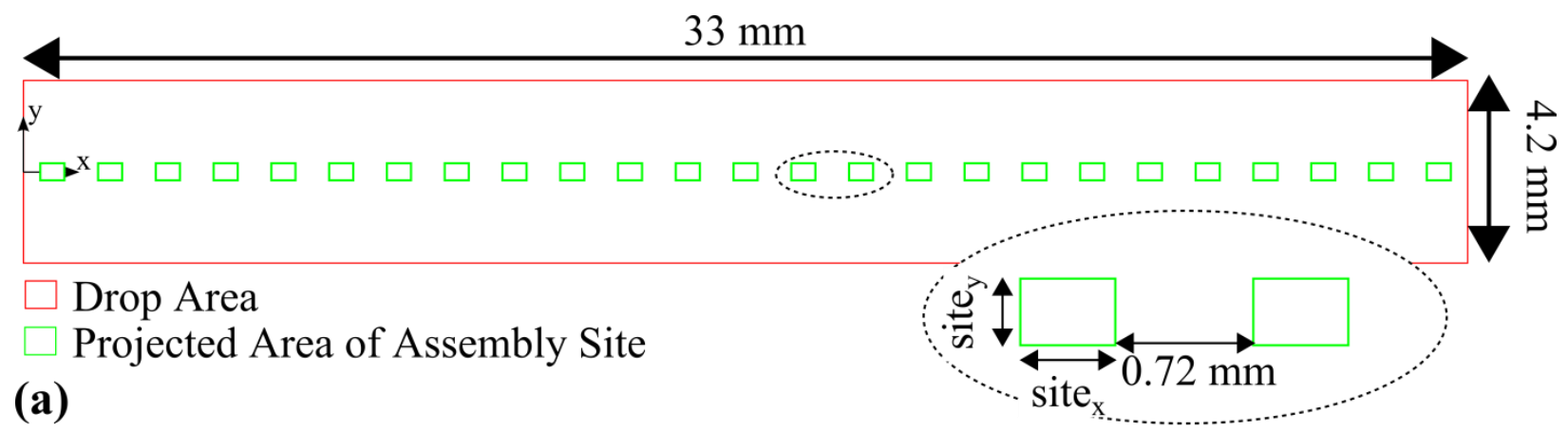

$33 \mathrm{~mm}$

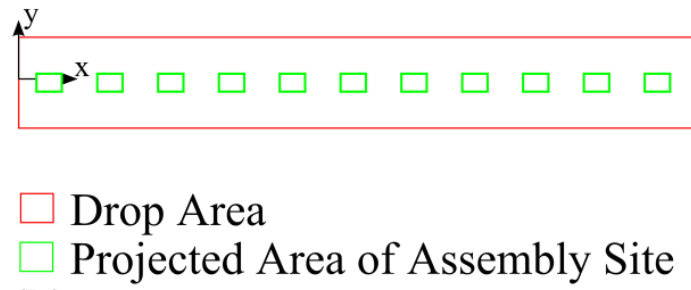

(b)

Projected Area of Assembly Site

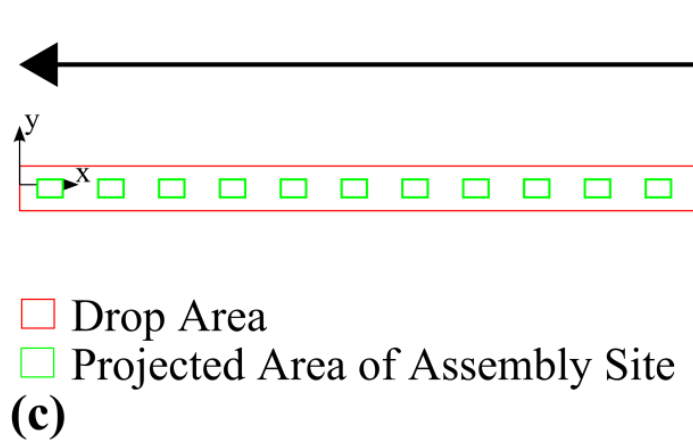

$33 \mathrm{~mm}$

Figure 37 Geometry for drop area and assembly site boundaries, implemented on $2^{\text {nd }}$ set (a) and $3^{\text {rd }}$ set (b) of Monte Carlo simulations for the study of assembly rate variation. The 1st simulation set implemented the geometry depicted in Figure 28.

At higher kinetic energies (which will be tested in future experiments), the tolerance for misalignments (thus, the correction factor) may likely be less than in previous low energy tests (where $\mathrm{c}=3$ ). Therefore, the assumption of $\rho_{a}=1$ with an uncorrected assembly site was implemented (i.e. $\mathrm{c}=1$ ). This assumption is not supported by experimentation. However, this study focuses on the variation produced by the varying rate of part-site interactions (i.e. on-site 
landings). The rate of interactions is independent of the probability of assembly, $\rho_{a}$. Therefore, the assumptions $\rho_{a}=1$ allowed a comparative analysis between drop areas, which only affect the rate of interactions, thus, it inherently affects the measured assembly rate.

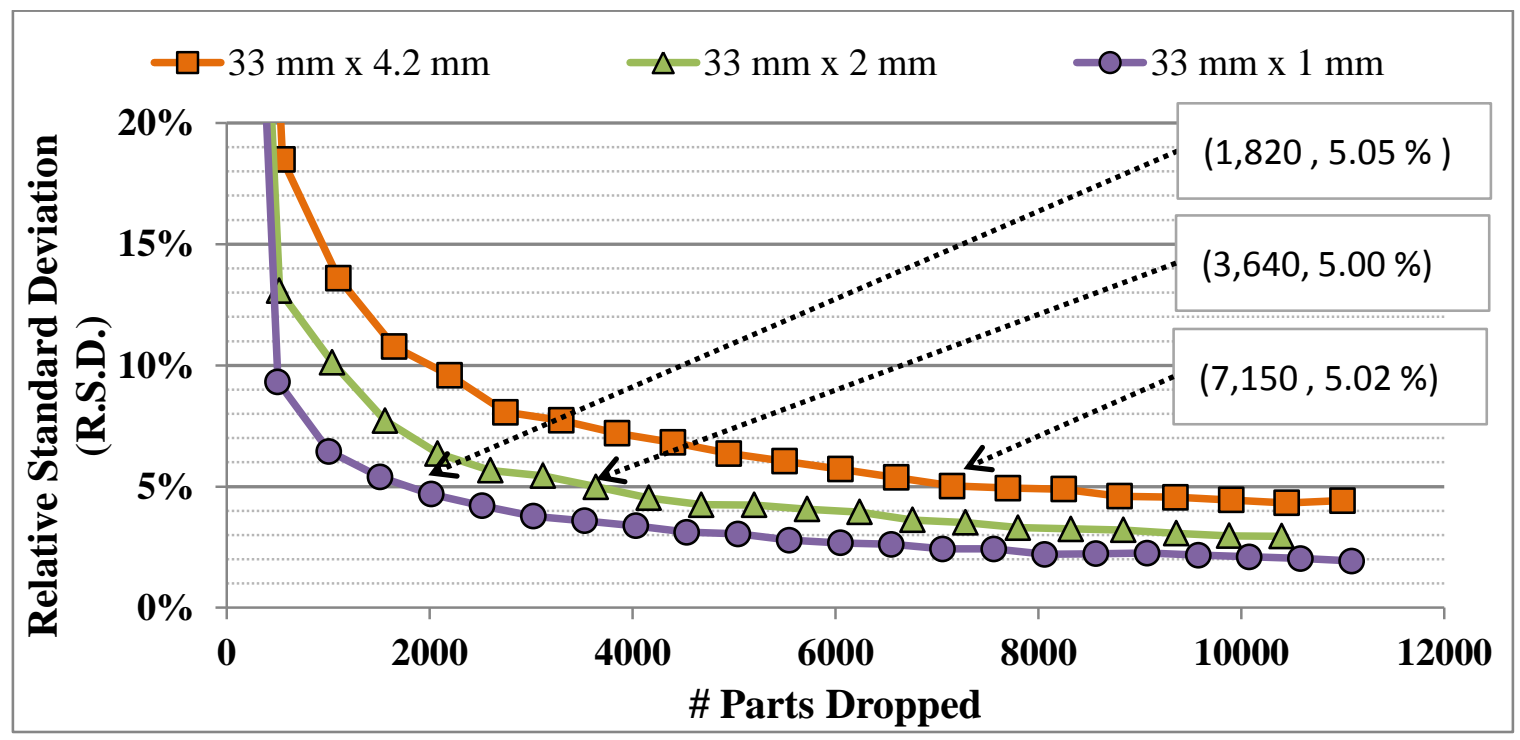

Figure 38 Progress of assembly rate as the number of parts dropped are incremented, for different drop area simulations. The $33 \mathrm{~mm}$ x $4.2 \mathrm{~mm}$ drop area was that of water-medium tests. The other two drop areas were decreased by approximately half in each subsequent set, in order to focus parts further.

Results from the simulations exploring different drop areas, at $\mathrm{c}=1$ are depicted in Figure 38. This plot displays the change of average assembly rate results with respect to the number of parts dropped. The main conclusion to extract from this plot is the minimum number of part drops required can be decreased for achieving an accepted low variation. Therefore, Table 7 summarizes the simulation results in terms of required number of part drops for achieving a variation with 5\% R.S.D. Simulations for $1^{\text {st }}$ drop area (plotted in Figure 38-a) achieve a R.S.D. 5\% after 7,950 part-drops. Smaller drop areas make it possible for dropping fewer parts and still achieve assembly rate R.S.D. below $5 \%$ (as evidenced by the Figure 38-b, c). These findings indicate that a higher drops density (i.e. smaller drop area for the same amount of drops) significantly increases the probability of on-site landings; therefore, it increases the rate at which 
part-site interactions occur. An increase in such rate increases an increase in assembly rate, which implies a higher signal-noise ratio

Table 7 Results of Monte Carlo simulation for different drop areas

\begin{tabular}{|c|c|c|c|c|c|}
\hline $\begin{array}{c}\text { Drop Area } \\
\left(\mathrm{mm}^{2}\right) \\
(\text { see Figure } 38)\end{array}$ & $\begin{array}{l}\text { Assembly } \\
\text { Site Area } \\
\left(\mathrm{mm}^{2}\right)\end{array}$ & $\begin{array}{c}\text { Average } \\
\text { Assembly Rate } \\
\left(\frac{\text { assemblies / sites }}{\text { parts dropped }}\right)\end{array}$ & $\begin{array}{l}\mathrm{N}^{\mathrm{o}} \text { part-drops } \\
(n) \text { required for } \\
5 \% \text { R.S.D. }\end{array}$ & $\begin{array}{c}\mathrm{N}^{\mathrm{O}} \\
\text { drops } \\
\text { per } \\
\text { strips }\end{array}$ & $\begin{array}{c}\mathrm{N}^{\mathrm{o}} \text { strips } \\
\text { required } \\
\text { for } 5 \% \\
\text { R.S.D. }\end{array}$ \\
\hline $33 \times 4.2$ & \multirow{3}{*}{$\begin{array}{c}0.6 \times \\
0.424 \\
(c=1)\end{array}$} & 0.0017 & $\approx 7,150$ & 55 & 130 \\
\hline $33 \times 2$ & & 0.0037 & $\approx 3,640$ & 26 & 140 \\
\hline $33 \times 1$ & & 0.0068 & $\approx 1,820$ & 14 & 130 \\
\hline
\end{tabular}

Table 7 indicates that the smallest drop area would achieve a desired variation R.S.D. = $5 \%$ with fewest number of part drops. However, the selected drop area must achieve a uniform uniformity across assembly sites. The proposed SA model (presented in Chapter 3) calculates assembly rate as $\dot{B}=\rho_{a} \cdot r_{i}$, where $r_{i}$ is the rate (or frequency) of interactions. The rate of interactions was approximated as the area fraction corresponding to assembly sites. Additionally, the measurements of assembly probability are sensitive to whether a part lands right over the center of the site, or instead lands near an edge. Hence, a uniform distribution of landing locations is required for averaging out the effect from these offsets on assembly probability. For these reasons, the revised strategy should achieve a uniform distribution of landing locations across the assembly site areas.

Figure 37 illustrated the $\mathrm{x}$ and $\mathrm{y}$ directions of an assembly site strip. While the uniformity along the $\mathrm{x}$-direction (length of the assembly strip) was not an issue for any case, attention was focused on the landing uniformity along the y direction (along the shortest dimension of the assembly strip). Figure 39-b shows three histogram plots from dropping 1,000,000 parts for each drop area tested above. Each area has a length of 33mm (along x direction), and a different width: $4.2 \mathrm{~mm}, 2 \mathrm{~mm}$ and $1 \mathrm{~mm}$ (along the y direction). The histograms reflect the distributions 
of landing locations along the y-direction, which are defined from the center of the assembly sites. Also, the boundaries of the assembly sites were illustrated by the red-dotted lines, for three different levels of misalignment tolerance (i.e. correction factors $c=3,=1 c$ and $c=0.25$ ). The distribution provided by the smallest drop area (i.e. $33 \mathrm{~mm}$ x $1 \mathrm{~mm}$ ) becomes considerably uneven for the $c=1$ and $c=3$ scenarios (see Figure 39-b). Hence, a smallest drop area of about $33 \mathrm{~mm} \times 2 \mathrm{~mm}$, is recommended for a revised SA test strategy. When running future experiments, the drop area should be optimized upon acquisition of initial data and more insight about the actual uncertainty in the drop locations.

Future experiments will evaluate the effects of energy. As the ratio $E_{k} / E_{b}$ increases, parts would not likely assemble when landing with much offset as they would with lower ratios. The amount of offset tolerance has been modeled by the scaling factor $c$, which signifies a change in the apparent assembly site area. Hence, additional simulations implemented different correction factors $(c=0.25, c=0.5, c=1$ and $c=3)$, and the selected drop area (33 $\mathrm{mm} \times 2 \mathrm{~mm})$. These allowed predicting different possible assembly rates that could become from changes in the energy ratio. Moreover, a smaller drop-area may become more adequate at low factors $c$. Accordingly, the smaller drop area $(33 \mathrm{~mm}$ x $1 \mathrm{~mm})$ was also simulated. Table 8 summarizes results from these simulations, portraying resulting average assembly rates and the number of part drops required for achieving R.S.D. $=5 \%$.

As shown in Table 8, increasing the apparent site area (which is an effect from a decreasing $E_{k} / E_{b}$ ratio) increases the frequency at which parts land on a site (which directly increases assembly rate). Consequently, such increase signifies less variation caused for a given number of parts dropped. Moreover, the varying assembly rate (caused by the change in scaling factor $c$ ) caused a change in the number of parts required for filling a single assembly strip at a 
consistent level. Section 5.5.1 offered the rationale for filling each assembly strip before filling $9.83 \%$ of its assembly sites. This allowed maximizing the amount of assembly data acquired from each strip before leaving a reasonable linear approximation. For this reason, the number of parts dropped in each strip was optimized at each simulated condition in order to consistently assemble roughly $9.8 \%$ of each strip.

(a)

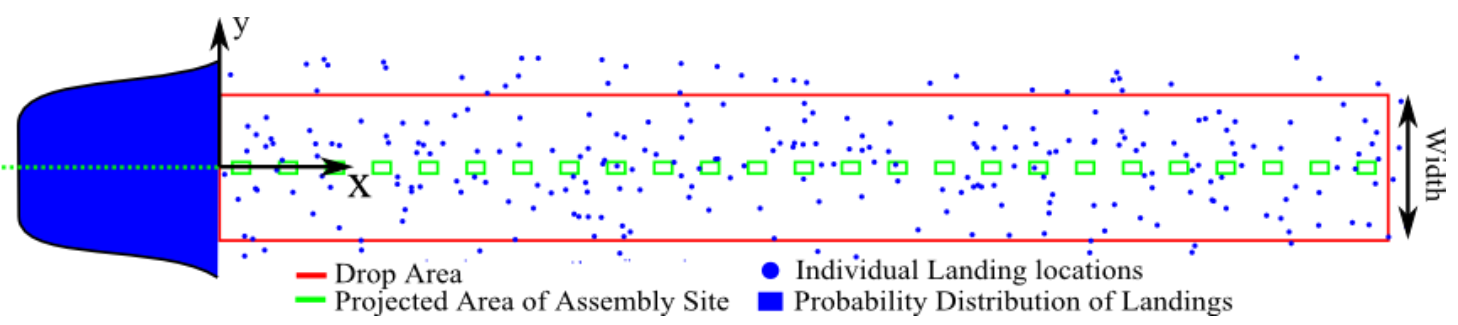

Drop Area width:

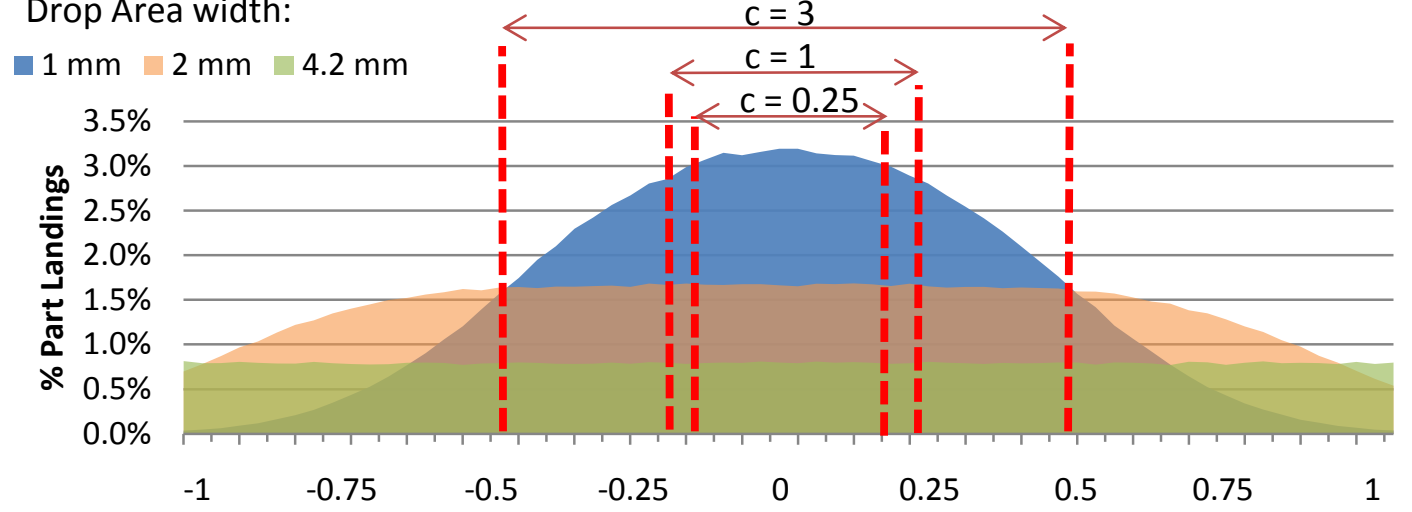

(b)

Distance away from assembly site center $(\mathrm{mm})$

Figure 39 Distribution of part landing locations for drop areas of different sizes. Simulations were performed to drop 1,000,000 parts from different drop areas, each with varying width: 4.2 $\mathrm{mm}$ (original size used in previous tests), $2 \mathrm{~mm}$ and $1 \mathrm{~mm}$ (see a). These drop area sizes were the same implemented in Monte Carlo simulations in Section 5.5 above. Simulations generated histogram plots of landing locations distribution along y-direction (see b).

Since the actual tolerance will not be known without initial test data, further analysis through Monte Carlo simulations should define a part number requirement. Additional simulations could be done to elucidate the impact of dropping different numbers of parts/strip. It may be possible to achieve lower RSD values for small values of 'c' by adjusting how many 
parts are dropped per strip. Nevertheless, the present simulations enlightened how much the sampling strategy is expected to change the outcomes.

Table 8 Results of Monte Carlo simulation for different misalignment tolerances

\begin{tabular}{|c|c|c|c|c|c|c|}
\hline $\begin{array}{c}\text { Drop } \\
\text { Area } \\
\left(\mathrm{mm}^{2}\right)\end{array}$ & $\begin{array}{c}\text { Correction } \\
\text { Factor* }\end{array}$ & $\begin{array}{c}\text { Assembly } \\
\text { site area } \\
\left(\mathrm{mm}^{2}\right)\end{array}$ & $\begin{array}{c}\text { Average } \\
\text { Assembly Rate } \\
\left(\frac{\text { assemblies } / \text { sites }}{\text { parts dropped }}\right)\end{array}$ & $\begin{array}{c}\mathrm{N}^{\mathrm{o}} \text { part-drops } \\
(n) \text { required } \\
\text { for 5\% } \\
\text { R.S.D. }\end{array}$ & $\begin{array}{c}\mathrm{N}^{\mathrm{o}} \text { drops } \\
\text { per strip }\end{array}$ & $\begin{array}{c}\mathrm{N}^{\mathrm{o}} \text { strips } \\
\text { required } \\
\text { for 5\% } \\
\text { R.S.D. }\end{array}$ \\
\hline \multirow{3}{*}{$33 \times 2$} & $\mathrm{c}=0.25$ & $0.3 \times 0.212$ & 0.0009 & 16,500 & 100 & 165 \\
\cline { 2 - 7 } & $\mathrm{c}=0.5$ & $0.424 \times 0.3$ & 0.0018 & 7,250 & 50 & 145 \\
\cline { 2 - 7 } & $\mathrm{c}=1$ & $0.6 \times 0.424$ & 0.0037 & 3,640 & 26 & 140 \\
\cline { 2 - 7 } & $\mathrm{c}=3$ & $0.6 \times 0.954$ & 0.0083 & 1,375 & 11 & 125 \\
\hline \multirow{3}{*}{$33 \times 1$} & $\mathrm{c}=0.25$ & $0.3 \times 0.212$ & 0.0017 & 8,100 & 54 & 150 \\
\cline { 2 - 7 } & $\mathrm{c}=0.5$ & $0.424 \times 0.3$ & 0.0035 & 3,861 & 27 & 143 \\
\cline { 2 - 7 } & $\mathrm{c}=1$ & $0.6 \times 0.424$ & 0.0068 & 1,820 & 14 & 130 \\
\cline { 2 - 7 } & $\mathrm{c}=3$ & $0.6 \times 0.954$ & 0.0135 & 750 & 7 & 107 \\
\hline
\end{tabular}

* Misalignment tolerance is quantified by the correction factor $c$, which is a multiplier of the actually assembly site area, defining the apparent assembly site area which provides assembly. This concept was introduced in Chapter 4.

\subsubsection{Implementing New Strategy to Future SA Experiments}

The revised strategy for creating SA interactions should target a low intrinsic variation for assembly rate measurements. Future SA experiments should implement the revised drop-area geometry (Figure 37-c). Moreover, a preliminary assumption of $\mathrm{c}=1$ allows preliminary testing to measure an average assembly rate. According to Monte Carlo simulations, dropping approximately 1,700 parts (corresponding to dropping 15 parts on each of 114 strips) under such conditions would achieve enough samples for a reduce R.S.D. below 5\%.

Future SA experiments should target this amount of sampling. If the average measurement is result different as expected, and even varies with higher standard deviations than expected, further simulations shall help improve the sampling strategy by adjusting the minimum sampling (number of part-drops) requirement. Moreover, the issue of landing locations 
uniformity must also be monitored. If the misalignment tolerance is larger, then a larger drop area may be required to maintain a desired uniformity.

\subsection{Conclusions}

Low sampling corresponded to the low number of part drops performed in each SA test, and also a low number of test trials for every configuration. These conditions caused an inconsistency in on-site landings, which yielded scatter in assembly rate data. The low sampling conditions from water-medium experiments caused a variation of assembly rate amounted to R.S.D. values between 5\% and 30\%. After 1,000 Monte Carlo simulation trials under the same conditions as water-medium experiments, variation was calculated with R.S.D. $=12.7 \%$. Therefore, the present Monte Carlo study confirmed that the noise seen on previous SA experiments was largely due to low sampling effects.

Moreover, the original test strategy caused many part-drops to fall on empty spaces; hence, it did not allow for creating sufficient sampling (i.e. dropping a required minimum number of parts) before filling up all assembly sites. Therefore, a revised strategy involved dropping parts on multiple strips of assembly parts. This approach allowed for creating as many assemblies as required to reduce the estimated R.S.D. to 5\%, without filling up all the assembly sites available. The revised strategy involves acquiring assembly data after dropping 15 parts on each strip, and repeating data acquisition over 114 strips. However, the strategy shall be revised (with further simulations) after gaining more insight about the effects of higher kinetic energies (or of other parameters tested). 


\section{CHAPTER 6:}

\section{CONCLUSIONS}

SA offers advantages over traditional micro-assembly methods. Being a parallel process, driven by external force fields, SA has the potential for offering much higher throughput and eliminates the need for expensive manipulation systems. Previous demonstrations of SA systems have been successful for specific applications and conditions. However, SA still encounters a different set of limitations. One main obstacle is that process requirements of microscale SA are dramatically more constraining than those of grasp and release methods. Moreover, the main limitation is that few works have provided guidance for general design of SA systems. Hence, there is a current need for tools that facilitate design and implementation of SA. This limitation is addressed by modeling efforts. The present study offers unprecedented input, and also offers significant contributions the complement the existing set of modeling efforts.

SA Process models focus on predicting process performance by, most commonly, calculating process rate and yield. Such models are a powerful tool for optimizing SA performance by varying process-level parameters, such as component quantities and workspace volume. However, existing models have relied on experimental data, in order to simplify the effects of the component-level parameters (such as the part speed and geometry, and the tolerance for misalignment of assembly bonds). Hence, these works do not offer any insight on how these component-level parameters can control the outcome of every SA interaction. The present study is most relevant to some modeling efforts (Massimo Mastrangeli et al., 2010; Mermoud et al., 2009), offering an experimental framework for relating the misalignment 
tolerance value to controllable parameters, such as component geometry, and bonding mechanism. The outcome from this work complements existing process models, towards creating a valuable design tool for implementing SA process.

The present work proposes a parametric model that defines the outcome of a basic SA interaction. A basic interaction is defined by one part approaching one site. The outcome of each interaction is defined by whether an assembly is produced. The parameters controlling such outcome were hypothesized to be: the ratio of kinetic energy $E_{k}$ over binding energy $E_{b}\left(E_{k} / E_{b}\right)$, component orientation $\rho_{o}$, and angle of incidence $\varphi$. This basic model does not account for more complex interactions, such as those where more than one part interacts simultaneously with one site, or where part motion is interrupted by previous collisions. However, most complex interactions can be broken into a simpler interaction in terms of the process variables studied here. The goal was to provide initial understanding of the basic assembly event, which could then be expanded towards more complex studies.

A SA experimental system was developed in order to control key parameters involved in an SA interaction. Chapter 3 described all the procedures for achieving independent control of each parameter, and environmental conditions. Moreover, tests relied on an automated pick-anddrop system. This experimental system was essential for creating repeatable part-site interactions. Through the SA experimental system, SA tests studied the effects of $\varphi$ and $\rho_{o}$ under a low energy ratio, $E_{k} / E_{b}(<0.1)$. This condition allowed for reducing energy effects significantly. Hence, interactions were created inside a water-medium so that they could fall at low and constant (terminal) velocity. Next experiments for studying energy effects were designed in an air-medium, allowing for evaluating a broad range of energy ratio $E_{k} / E_{b}$ values, from just above 0 , to beyond 1 . For such medium, a vacuum gripper was installed on the pick- 
drop system. For a water medium, the pick-drop system relied on a capillary gripper. Results from these experiments were presented in Chapter 4.

\subsection{Key Conclusions}

Effects from the angle of incidence were significantly smaller than the effects from partgeometry. While there may be some path dependency, SA could be modeled as an energy-based (path-independent) process. Such simplification would prove useful for a predictive model. The part geometry effects were directly related to the probability of orientation. Such effects were clear, and even higher than expected for basic part-site interactions. These results confirm that part geometry must be considered when designing a Self-Assembly process.

Results provided unprecedented insight into the physics of part site interactions, and complement previous process modeling efforts (Massimo Mastrangeli et al., 2010). This agentbased model captures valuable predictions about the rate of SA interactions, and how it is affected by process-level parameters. Moreover, the model simulates agitation generated to components, and tracks the dynamics of such components. Hence, the kinetic energy, motion and orientation could be simulated. The present experimental model would then add to these agent based model by using these tracked parameter values as input, and predicting the probability of assembly that each interaction would have.

Direct observation of the experimental interactions evidenced that besides basic interactions, a secondary type of interactions was present. These secondary interactions occurred when parts landed above a site, and then slid or rolled on to assemble. The model accounted for such interactions by a considering a tolerance for misalignment. This tolerance was represented by an increase in the effective assembly site area, quantified by a correction factor (c) that multiplied the actual area. This tolerance for misalignment is also due to the alignment forces of 
the bonding liquid. The tolerance for misalignment is hypothesized to be dependent on kinetic energy. For higher $E_{k} / E_{b}$ ratios, the tolerance is expected to decrease. Therefore, a more detailed focus on this concept will be in the scope of energy-study experiments.

Results from water-medium experiments showed a significant amount of data scatter. While clear trends were still recognizable, the noise prevented from creating regression fits with high confidence. For this reason, Chapter 5 focused on investigated a main contributor to such noise. A Monte-Carlo study confirmed that confirmed that the noise seen on previous SA experiments was largely due to effects of a low number of samples. Low sample numbers caused an inconsistency in on-site landings, which yielded scatter in assembly rate data. The low sampling conditions from water-medium experiments caused a variation of assembly rate amounted to relative standard deviation (R.S.D.) values between 5\% and 30\%.. Moreover, Monte-Carlo simulations offered valuable information about how to achieve a desired reduction in SA data variation. For this purpose, modifications in the assembly site geometry, and sampling strategy were required.

\subsection{Future Work}

Additional work is required to study the energy effects, and confirm the adequacy of the energy-based model. The energy ratio as an assembly parameter is expected to not only affect the outcome of a direct part-site interaction without misalignment, but also affect the tolerance for misalignment. The revised strategy generated by the Monte Carlo simulations shall be implemented in these tests. Such strategy is expected to provide significant reduction of previously-seen data scatter, and provide high-confidence regression fits providing a relation between energy effects and SA interactions. Higher energy levels will be achieved by dropping parts in air with varying heights from the part sites. 
The systems that were developed for this work can also be used to evaluate other important process criteria such as the volume of the fluid on the drop sites. As the fluid volume increases, the droplet protrudes above the site and is more likely to make contact, but the stiffness of the bond is expected to decrease. This could negatively impact the assembly accuracy. These tradeoffs are important to the design of practical assembly systems and should be addressed.

It would also be helpful to apply the testing strategy developed in this project using another bond type such as magnetic or electrostatic forces. Capillary bonds have impactabsorption capabilities - as they are formed by liquids - that other bonding types do not have. Consequently, the assembly rate of capillary based SA would be less sensitive to high kinetic energies. A future completion of this parametric model shall include these effects from impactabsorption. Such effects could be modeled as a scalar that amplifies the function of $E_{k} / E_{b}$; or instead they likely deserve a modified separate $E_{k} / E_{b}$ function (if effects are non-linear). Nevertheless, the results from this work provide valuable insight not just on the specific bonding method, but also provide insight into how well the self-assembly model and the test methods for evaluating it can be generalized to other self-assembly methods. 


\section{REFERENCES}

Arutinov, G., Mastrangeli, M., Smits, E. C. P., Schoo, H. F. M., Brugger, J., \& Dietzel, A. (2013). Dynamics of capillary self-alignment for mesoscopic foil devices. Applied Physics Letters, 102(14), 144101. doi:10.1063/1.4801088

Bassik, N., Stern, G. M., \& Gracias, D. H. (2009). Microassembly based on hands free origami with bidirectional curvature. Applied Physics Letters, 95(9), 91901. doi:10.1063/1.3212896

Berthier, J., Brakke, K., Grossi, F., Sanchez, L., \& Di Cioccio, L. (2010). Self-alignment of silicon chips on wafers: A capillary approach. Journal of Applied Physics, 108(5), 054905. doi:10.1063/1.3466782

Biebuyck, H. a., \& Whitesides, G. M. (1994). Self-Organization of Organic Liquids on Patterned Self-Assembled Monolayers of Alkanethiolates on Gold. Langmuir, 10(8), 2790-2793. doi:10.1021/la00020a047

Bohringer, K. F., Srinivasan, U., \& Howe, R. T. (2001). Modeling of capillary forces and binding sites for fluidic self-assembly. In Technical Digest. MEMS 2001. 14th IEEE International Conference on Micro Electro Mechanical Systems (Cat. No.01CH37090) (pp. 369-374). Ieee. doi:10.1109/MEMSYS.2001.906555

Bohringer, K.-F., Goldberg, K., Cohn, M., Howe, R., \& Pisano, A. (1998). Parallel microassembly with electrostatic force fields. Proceedings. 1998 IEEE International Conference on Robotics and Automation (Cat. No.98CH36146), (May), 1204-1211. doi:10.1109/ROBOT.1998.677259

Bouleau, N., \& Lépingle, D. (1994). Numerical methods for stochastic processes. New York, NY: Wiley.

Bowden, N., Terfort, A., Carbeck, J., \& Whitesides, G. M. (1997). Self-Assembly of Mesoscale Objects into Ordered Two-Dimensional Arrays. Science, 276(5310), 233-235. doi:10.1126/science.276.5310.233

Brakke, K. (1992). The Surface Evolver. Exp. Math, 1, 141.

Breen, T. L. (1999). Design and Self-Assembly of Open, Regular, 3D Mesostructures. Science, 284(5416), 948-951. doi:10.1126/science.284.5416.948 
Carballo, J. M., \& Crane, N. B. (2014). Self-Assembly Kinetics of Microscale Components: A Parametric Evaluation. Journal of Microelectromechanical Systems, 1-1.

doi:10.1109/JMEMS.2014.2354137

Carlisle, B. (2009). Flexible and Precision Assembly. In S. Y. Nof (Ed.), Springer Handbook of Automation (pp. 881-891). Berlin, Heidelberg: Springer Berlin Heidelberg. doi:10.1007/978-3-540-78831-7

Chen, B. K., \& Sun, Y. (2013). Miniaturization of Micromanipulation Tools. In Advanced Mechatronics and MEMS Devices (pp. 89-98). New York: Springer New York. doi:10.1007/978-1-4419-9985-6_4

Chung, J., Zheng, W., Hatch, T. J., \& Jacobs, H. O. (2006). Programmable Reconfigurable SelfAssembly: Parallel Heterogeneous Integration of Chip-Scale Components on Planar and Nonplanar Surfaces. Journal of Microelectromechanical Systems, 15(3), 457-464. doi:10.1109/JMEMS.2006.872226

Chung, S. E., Park, W., Shin, S., Lee, S. A., \& Kwon, S. (2008). Guided and fluidic selfassembly of microstructures using railed microfluidic channels. Nature Materials, 7(7), 581-7. doi:10.1038/nmat2208

Cohn, M. B., Böhringer, K. F., Noworolski, J. M., Singh, A., Keller, C. G., Goldberg, K. Y., ... Bldg, E. E. C. S. E. (1998). Microassembly Technologies for MEMS. In Proceedings of SPIE Micromachining and Microfabrication. Santa Clara, CA.

Crane, N. B., Mishra, P., Murray, J. L., \& Nolas, G. S. (2009). Self-Assembly for Integration of Microscale Thermoelectric Coolers. Journal of Electronic Materials, 38(7), 1252-1256. doi:10.1007/s11664-008-0627-9

Crane, N. B., Onen, O., Carballo, J., Ni, Q., \& Guldiken, R. (2013). Fluidic assembly at the microscale: progress and prospects. Microfluidics and Nanofluidics, 14(3), 383-419. doi:10.1007/s10404-012-1060-1

Crane, N. B., Tuckerman, J., \& Nielson, G. N. (2011). Self-assembly in additive manufacturing: opportunities and obstacles. Rapid Prototyping Journal, 17(3), 211-217. doi:10.1108/13552541111124798

Crane, Nathan B.; Mcknight, P. (2012). Impact of Self-Assembly Process Errors on Thermoelectric Performance. Journal of Electronic Packaging, 134(3), 1-7.

Fang, J., \& Bohringer, K. F. (2006). Wafer-Level Packaging Based on Uniquely Orienting SelfAssembly (The DUO-SPASS Processes). Journal of Microelectromechanical Systems, 15(3), 531-540. doi:10.1109/JMEMS.2006.876790

Fang, J., \& Böhringer, K. F. (2008). 1.14 - Self-Assembly. In H. B. T. Zappe (Ed.), (pp. 403429). Oxford: Elsevier. doi:http://dx.doi.org/10.1016/B978-044452190-3.00018-5 
Fang, J., Wang, K., \& Bohringer, K. F. (2006). Self-Assembly of PZT Actuators for Micropumps With High Process Repeatability. Journal of Microelectromechanical Systems, 15(4), 871878. doi:10.1109/JMEMS.2006.878880

Fearing, R. S. (1995). Survey of sticking effects for micro parts handling. In Proceedings 1995 IEEE/RSJ International Conference on Intelligent Robots and Systems. Human Robot Interaction and Cooperative Robots (Vol. 2, pp. 212-217). IEEE Comput. Soc. Press. doi:10.1109/IROS.1995.526162

Fonstad, C. G. (2002). Magnetically-Assisted Statistical Assembly - a New Heterogeneous Integration Technique. Boston, MA.

Gauthier, M., \& Regnier, S. (2010). Robotic Microassembly. Wiley-Blackwell (an imprint of John Wiley \& Sons Ltd).

Golosovsky, M., Saado, Y., \& Davidov, D. (1999). Self-assembly of floating magnetic particles into ordered structures: A promising route for the fabrication of tunable photonic band gap materials. Applied Physics Letters, 75(26), 4168. doi:10.1063/1.125571

Gracias, D. H., Boncheva, M., Omoregie, O., \& Whitesides, G. M. (2002). Biomimetic selfassembly of helical electrical circuits using orthogonal capillary interactions. Applied Physics Letters, 80(15), 2802. doi:10.1063/1.1470222

Gracias, D. H. D., Tien, J., Breen, T., Hsu, C., \& Whitesides, G. (2000). Forming Electrical Networks in Three Dimensions by Self-Assembly. Science, 289(5482), 1170-1172. doi:10.1126/science.289.5482.1170

Greiner, A., Lienemann, J., Korvink, J. G., Xiong, X., Hanein, Y., \& Böhringer, K. F. (2002). Capillary forces in micro-fluidic self-assembly. In Fifth International Conference on Modeling and Simulation of Microsystems (MSM'02) (pp. 22-25).

Grzybowski, B. A., Stone, H. A., \& Whitesides, G. M. (2000). Dynamic self-assembly of objects rotating at a liquid air interface. Nature, 405(June), 1033-1036.

Harsh, K., \& Lee, Y. (1998). Modeling for solder self-assembled MEMS. Optoelectronics and High- ..., 3289, 177-184.

Hendrick, G. (2010). Modeling and Experimentation of Micro-Scale Self-Assembly Processes. Graduate Theses and Dissertations. University of South Florida.

Hofmann, A. (2010). A Comprehensive Micro-assembly Process Oriented Methodology Supporting the Realisation of Evolvable Micro Production Systems. In G. Huang, K. L. Mak, \& P. Maropoulos (Eds.), Proceedings of the 6th CIRP-Sponsored International Conference on Digital Enterprise Technology SE - 64 (Vol. 66, pp. 823-839). Springer Berlin Heidelberg. doi:10.1007/978-3-642-10430-5_64 
Hosokawa, K., Shimoyama, I., \& Miura, H. (1994). Dynamics of Self-Assembling Systems: Analogy with Chemical Kinetics. Artificial Life, 1(4), 413-427. doi:10.1162/artl.1994.1.4.413

Hosokawa, K., Shimoyama, I., \& Miura, H. (1996). Two-dimensional micro-self-assembly using the surface tension of water. Sensors and Actuators A: Physical, 57(2), 117-125. doi:10.1016/S0924-4247(97)80102-1

Houston, P. L. (2001). The Rate of Chemical Reactions. In Chemical kinetics and reaction dynamics (1st ed.). Boston: McGraw-Hill.

Jacobs, H. O., Tao, A. R., Schwartz, A., Gracias, D. H., \& Whitesides, G. M. (2002). Fabrication of a cylindrical display by patterned assembly. Science (New York, N.Y.), 296(5566), 323-5. doi:10.1126/science.1069153

Kataria, A., \& Rosen, D. W. (2001). Building around inserts: methods for fabricating complex devices in stereolithography. Rapid Prototyping Journal, 7(5), 253-262. doi:10.1108/13552540110410459

Knuesel, R. J., \& Jacobs, H. O. (2010). Self-assembly of microscopic chiplets at a liquid-liquidsolid interface forming a flexible segmented monocrystalline solar cell. Proceedings of the National Academy of Sciences of the United States of America, 107(3), 993-998.

Knuesel, R. J., \& Jacobs, H. O. (2011). Self-tiling monocrystalline silicon; a process to produce electrically connected domains of $\mathrm{Si}$ and microconcentrator solar cell modules on plastic supports. Advanced Materials (Deerfield Beach, Fla.), 23(24), 2727-33. doi:10.1002/adma.201004533

Knuesel, R., \& Park, S. (2012). Self-assembly and self-tiling: integrating active dies across length scales on flexible substrates. Systems, Journal of, 21(1), 85-99.

Langmuir, I. (1938). The Role of Attractive and Repulsive Forces in the Formation of Tactoids, Thixotropic Gels, Protein Crystals and Coacervates. The Journal of Chemical Physics, 6, 873. doi:http://dx.doi.org/10.1063/1.1750183

Liu, M., Lau, W. M., \& Yang, J. (2007). On-demand multi-batch self-assembly of hybrid MEMS by patterning solders of different melting points. Journal of Micromechanics and Microengineering, 17(11), 2163-2168. doi:10.1088/0960-1317/17/11/001

Lu, Y., Xia, S., Liu, M., \& Zhang, J. (2006). Dynamic Simulation of MEMS Self-Assembly Using Capillary Force. 2006 1st IEEE International Conference on Nano/Micro Engineered and Molecular Systems, 414-417. doi:10.1109/NEMS.2006.334788 
Mastrangeli, M., Abbasi, S., Varel, C., Van Hoof, C., Celis, J.-P., \& Böhringer, K. F. (2009). Self-assembly from milli- to nanoscales: methods and applications. Journal of Micromechanics and Microengineering : Structures, Devices, and Systems, 19(8). doi:10.1088/0960-1317/19/8/083001

Mastrangeli, M., Mermoud, G., \& Martinoli, A. (2011). Modeling Self-Assembly Across Scales: The Unifying Perspective of Smart Minimal Particles. Micromachines, 2(4), 82-115. doi: $10.3390 / \mathrm{mi} 2020082$

Mastrangeli, M., Ruythooren, W., Van Hoof, C., \& Celis, J.-P. (2009). Conformal dip-coating of patterned surfaces for capillary die-to-substrate self-assembly. Journal of Micromechanics and Microengineering, 19(4), 045015. doi:10.1088/0960-1317/19/4/045015

Mastrangeli, M., Van Hoof, C., Baskaran, R., Celis, J.-P., \& Bohringer, K. F. (2010). Agentbased modeling of mems fluidic self-assembly. 2010 IEEE 23rd International Conference on Micro Electro Mechanical Systems (MEMS), 476-479.

doi:10.1109/MEMSYS.2010.5442463

Maybeck. (1979). Stochastic Models: Estimation and Control. v.1 (1st ed.). Oxford: Elsevier Science.

McNally, H., Pingle, M., \& Lee, S. W. (2003). Self-assembly of micro-and nano-scale particles using bio-inspired events. Applied Surface, 214(1-4), 109-119. doi:10.1016/S01694332(03)00266-6

Mermoud, G., Brugger, J., \& Martinoli, A. (2009). Towards multi-level modeling of selfassembling intelligent micro-systems. In Proceedings of The 8th International Conference on Autonomous Agents and Multiagent Systems (pp. 89-96). Budapest: International Foundation for Autonomous Agents and Multiagent Systems.

Morris, C. J., \& Parviz, B. A. (2008). Micro-scale metal contacts for capillary force-driven selfassembly. Journal of Micromechanics and Microengineering, 18(1).

Morris, C. J., Stauth, S. a., \& Parviz, B. a. (2005). Self-assembly for microscale and nanoscale packaging: steps toward self-packaging. IEEE Transactions on Advanced Packaging, 28(4), 600-611. doi:10.1109/TADVP.2005.858454

Napp, N., Burden, S., \& Klavins, E. (2006). The statistical dynamics of programmed selfassembly. Robotics and Automation, 2006., 2006, 1469-1476.

O'Riordan, A., Delaney, P., \& Redmond, G. (2004). Field Configured Assembly: Programmed Manipulation and Self-assembly at the Mesoscale. Nano Letters, 4(5), 761-765. doi: $10.1021 / \mathrm{nl} 1034145 \mathrm{q}$ 
Oliver, S. R. J., Clark, T. D., Bowden, N., \& Whitesides, G. M. (2001). Three-Dimensional SelfAssembly of Complex, Millimeter-Scale Structures through Capillary Bonding. Journal of the American Chemical Society, 123(33), 8119-8120. doi:10.1021/ja010845q

Onoe, H., Matsumoto, K., \& Shimoyama, I. (2004). Three-Dimensional Micro-Self-Assembly Using Hydrophobic Interaction Controlled by Self-Assembled Monolayers. Journal of Microelectromechanical Systems, 13(4), 603-611. doi:10.1109/JMEMS.2004.832183

Park, K. S., Xiong, X., Baskaran, R., \& Böhringer, K. F. (2011). Mechanics and scaling of thin part assembly at a fluidic interface. Journal of Micromechanics and Microengineering, 21(2), 025002. doi:10.1088/0960-1317/21/2/025002

Pelesko, J. A. (2007). Self Assembly: The Science of Things That Put Themselves Together. Hoboken: Taylor.

Ramadan, Q., Uk, Y. S., \& Vaidyanathan, K. (2007). Large scale microcomponents assembly using an external magnetic array. Applied Physics Letters, 90(17), 172502. doi:10.1063/1.2731708

Ramadoss, V., \& Crane, N. B. (2008). Design of fluidic self-assembly bonds for precise component positioning. In Photonics Packaging, Integration, and Interconnects VIII (Vol. 6899, p. 68990). San Jose, CA, United States: SPIE, Bellingham WA, WA 98227-0010, United States.

Sharma, R. (2007). Thermally controlled fluidic self-assembly. Langmuir: The ACS Journal of Surfaces and Colloids, 23(12), 6843-9. doi:10.1021/la063516q

Shet, S., Mehta, V. R., Fiory, A. T., Ravindra, N. M., \& Lepselter, M. P. (2004). The magnetic field-assisted assembly of nanoscale semiconductor devices: A new technique. JOM, 56(10), 32-34. doi:10.1007/s11837-004-0286-0

Sigma Aldrich. (2006). Preparing Self-Assembled Monolayers ( SAMs ) A Step-by-Step Guide for Solution-Based Self-Assembly.

Smid, P. (2008). CNC Programming Handbook. New York: Industrial Press.

Smith, J. S. (1994). Fluidic self-assembly of microstructures and its application to the integration of GaAs on Si. Proceedings IEEE Micro Electro Mechanical Systems An Investigation of Micro Structures, Sensors, Actuators, Machines and Robotic Systems, 279-284. doi:10.1109/MEMSYS.1994.555822

Smith, J. S. (2000). High density, low parasitic direct integration by fluidic self assembly (FSA). In Electron Devices Meeting, 2000. IEDM Technical Digest. International (pp. 201-204). IEEE. doi:10.1109/IEDM.2000.904292 
Srinivasan, U., Liepmann, D., \& Howe, R. T. (2001). Microstructure to substrate self-assembly using capillary forces. Journal of Microelectromechanical Systems, 10(1), 17-24. doi:10.1109/84.911087

Stauth, S. A., \& Parviz, B. A. (2006). Self-assembled single-crystal silicon circuits on plastic. Proceedings of the National Academy of Sciences of the United States of America, 103(38), 13922-7. doi:10.1073/pnas.0602893103

Terfort, A., Bowden, N., \& Whitesides, G. M. (1997). Three-dimensional self-assembly of millimetre-scale components. Nature, 386(6621), 162-164.

Tolley, M., Baisch, A., \& Krishnan, M. (2008). Interfacing methods for fluidically-assembled microcomponents. , 2008. MEMS 2008., 3-6.

Tolley, M., Krishnan, M., Erickson, D., \& Lipson, H. (2008). Dynamically programmable fluidic assembly. Applied Physics, 93(25), 254105.

Verma, A. K. F. self-assembly of silicon microstructures, Hadley, M. a., Yeh, H.-J. J., \& Smith, J. S. (1995). Fluidic self-assembly of silicon microstructures. In 1995 Proceedings. 45th Electronic Components and Technology Conference (pp. 1263-1268). Ieee. doi:10.1109/ECTC.1995.517853

Whitesides, G. M., \& Grzybowski, B. (2002). Self-Assembly at All Scales. Science, 295(5564), 2418-21. doi:10.1126/science.1070821

Whitesides, G. M., \& Laibinis, P. E. (1990). Wet chemical approaches to the characterization of organic surfaces: self-assembled monolayers, wetting, and the physical-organic chemistry of the solid-liquid interface. Langmuir, 6(1), 87-96. doi:10.1021/la00091a013

Wu, H., Bowden, N., \& Whitesides, G. M. (1999). Selectivities among capillary bonds in mesoscale self-assembly. Applied Physics Letters, 75(20), 3222. doi:10.1063/1.125284

Xiong, X., Hanein, Y., Fang, J., Wang, Y., Wang, W., Schwartz, D. T., \& Bohringer, K. F. (2003). Controlled multibatch self-assembly of microdevices. Journal of Microelectromechanical Systems, 12(2), 117-127. doi:10.1109/JMEMS.2003.809964

Xiong, X., Hanein, Y., Wang, W., Schwartz, D. T., \& Bohringer, K. F. (2001). Multi-batch micro-self-assembly via controlled capillary forces. In International Conference on Intelligent Robots and Systems (Vol. vol.3, pp. 1335-1342). Maui, HI, USA: IEEE.

Xiong, X., Liang, S.-H., \& Bohringer, K. (2004). Geometric binding site design for surfacetension driven self-assembly. IEEE International Conferenece on Robotics and Automation, $2,1141-1148$. 
Yeh, H.-J. J., \& Smith, J. S. (1994). Fluidic self-assembly for the integration of GaAs lightemitting diodes on Si substrates. IEEE Photonics Technology Letters, 6(6), 706-708. doi:10.1109/68.300169

Zhang, X., Chen, C.-C., Bernstein, R. W., Zappe, S., Scott, M. P., \& Solgaard, O. (2005). Microoptical characterization and modeling of positioning forces on drosophila embryos self-assembled in two-dimensional arrays. Journal of Microelectromechanical Systems, 14(5), 1187-1197. doi:10.1109/JMEMS.2005.851834

Zheng, W., \& Jacobs, H. O. (2004). Shape-and-solder-directed self-assembly to package semiconductor device segments. Applied Physics Letters, 85(16), 3635. doi:10.1063/1.1807017

Zheng, W., \& Jacobs, H. O. (2005). Fabrication of Multicomponent Microsystems by Directed Three-Dimensional Self-Assembly. Advanced Functional Materials, 15(5), 732-738. doi:10.1002/adfm.200400595 


\section{APPENDIX A:}

\section{PHOTOLITHOGRAPHY MASKS}

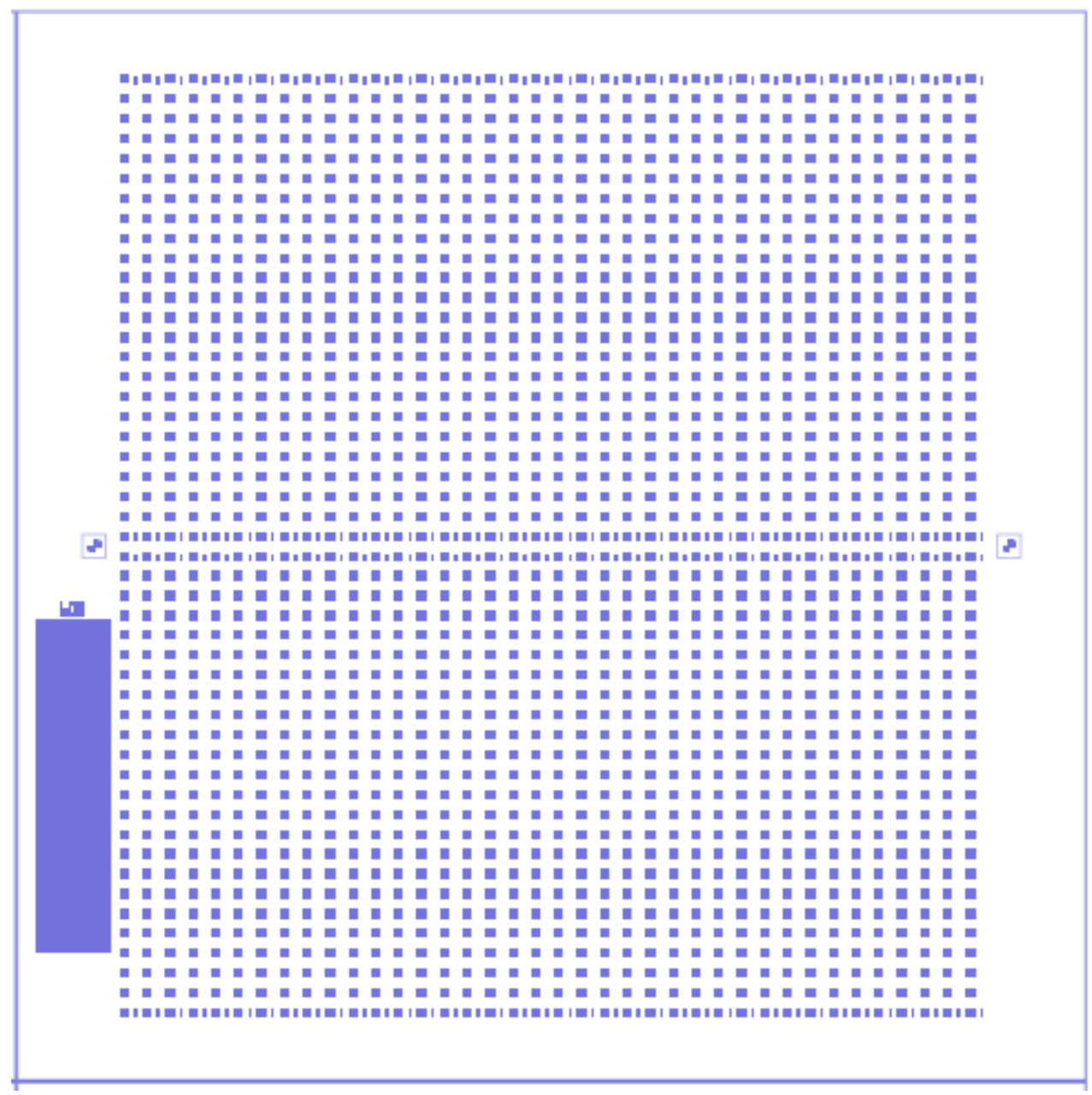

Figure A.1 Photolithography mask for water-medium test sites 


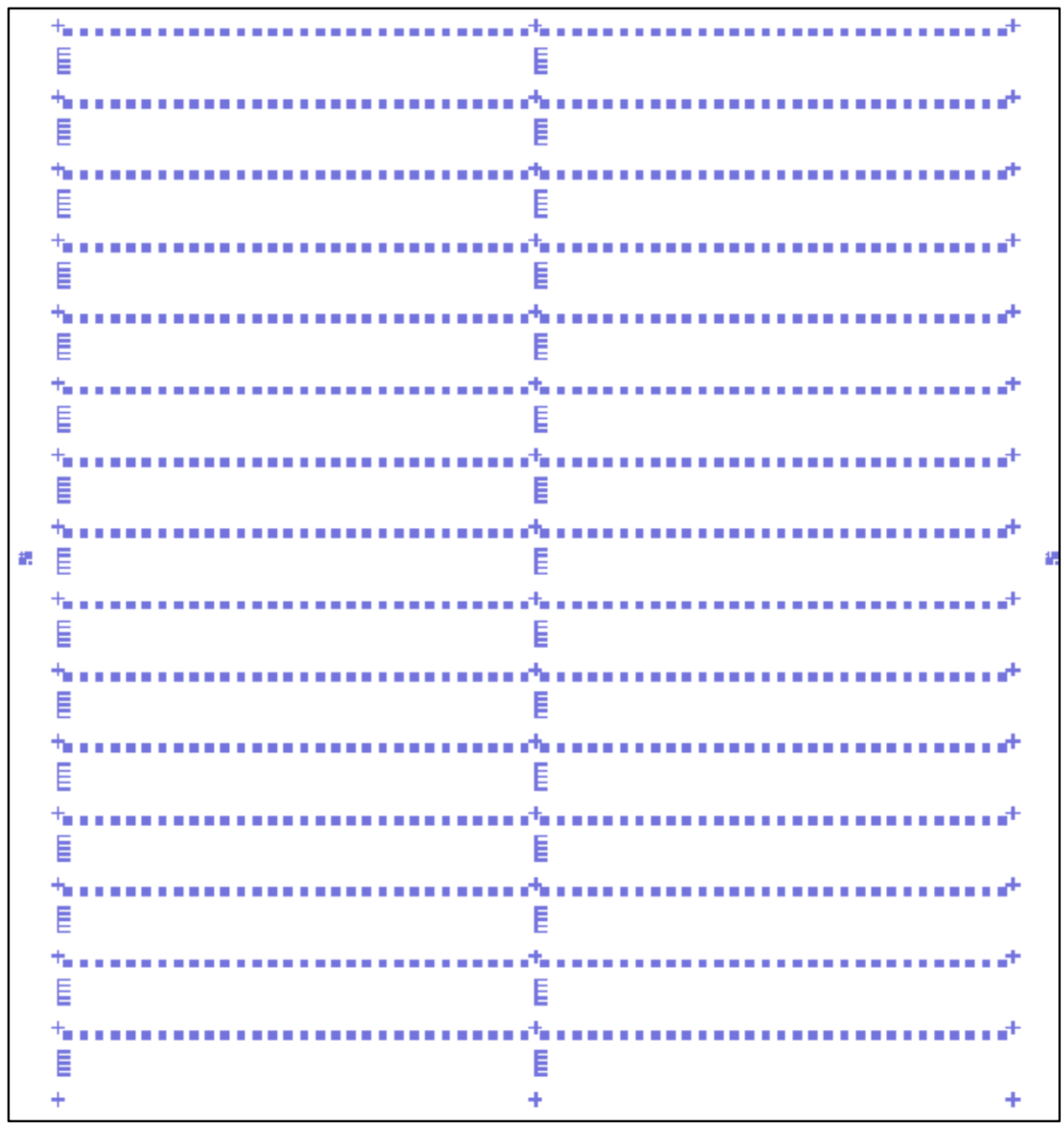

Figure A.2 Photolithography mask for air-medium test sites 


\section{APPENDIX B :}

\section{SAMPLE GCODES}

The following figure depicts a sample GCode program that was utilized for running the CNC machine, to work as a pick-and-drop system. It only utilizes basic GCode commands, for tool motion, and tool on/off switching. The reader is referred to online sources for more information about GCode programming syntax.

\begin{tabular}{|c|}
\hline G90 \\
G02 X0 Y0 Z0 Y0 Z0.2 \\
G0 X0 Y0 Z0.2 \\
G0 X0 Y0 Z0 \\
G1 X0 Y0 Z-0.03 F5 \\
G0 X0 Y0 Z0.2 \\
G0 X-1.790 Y-1.155 Z0.2 \\
G1 X-1.790 Y-1.155 Z-1.65 F140 \\
G1 X-1.790 Y-1.155 Z0.2 F140 \\
G0 X0.1404 Y-0.647 Z0.2 \\
G0 X0.1404 Y-0.647 Z0 \\
G1 X0.1404 Y-0.647 Z-0.03 F5 \\
G0 X0.1404 Y-0.647 Z0.2 \\
G0 X-1.677 Y-2.348 Z0.2 \\
G1 X-1.677 Y-2.348 Z-1.65 F140 \\
G1 X-1.677 Y-2.348 Z0.2 F140 \\
G0 X0.1477 Y-0.899 Z0.2 \\
G0 X0.1477 Y-0.899 Z0 \\
G1 X0.1477 Y-0.899 Z-0.03 F5 \\
G0 X0.1477 Y-0.899 Z0.2 \\
G0 X-2.640 Y-2.305 Z0.2 \\
G1 X-2.640 Y-2.305 Z-1.65 F140 \\
G1 X-2.640 Y-2.305 Z0.2 F140 \\
G0 X0.1560 Y-0.562 Z0.2 \\
G0 X0 Y0 Z0.2
\end{tabular}

Figure B.1 Sample GCode program for running CNC-driven pick-and-drop system 


\section{APPENDIX C:}

\section{MATLAB SCRIPTS}

\section{C.1 Matlab Script for Image Analysis and Part-Location Recognition}

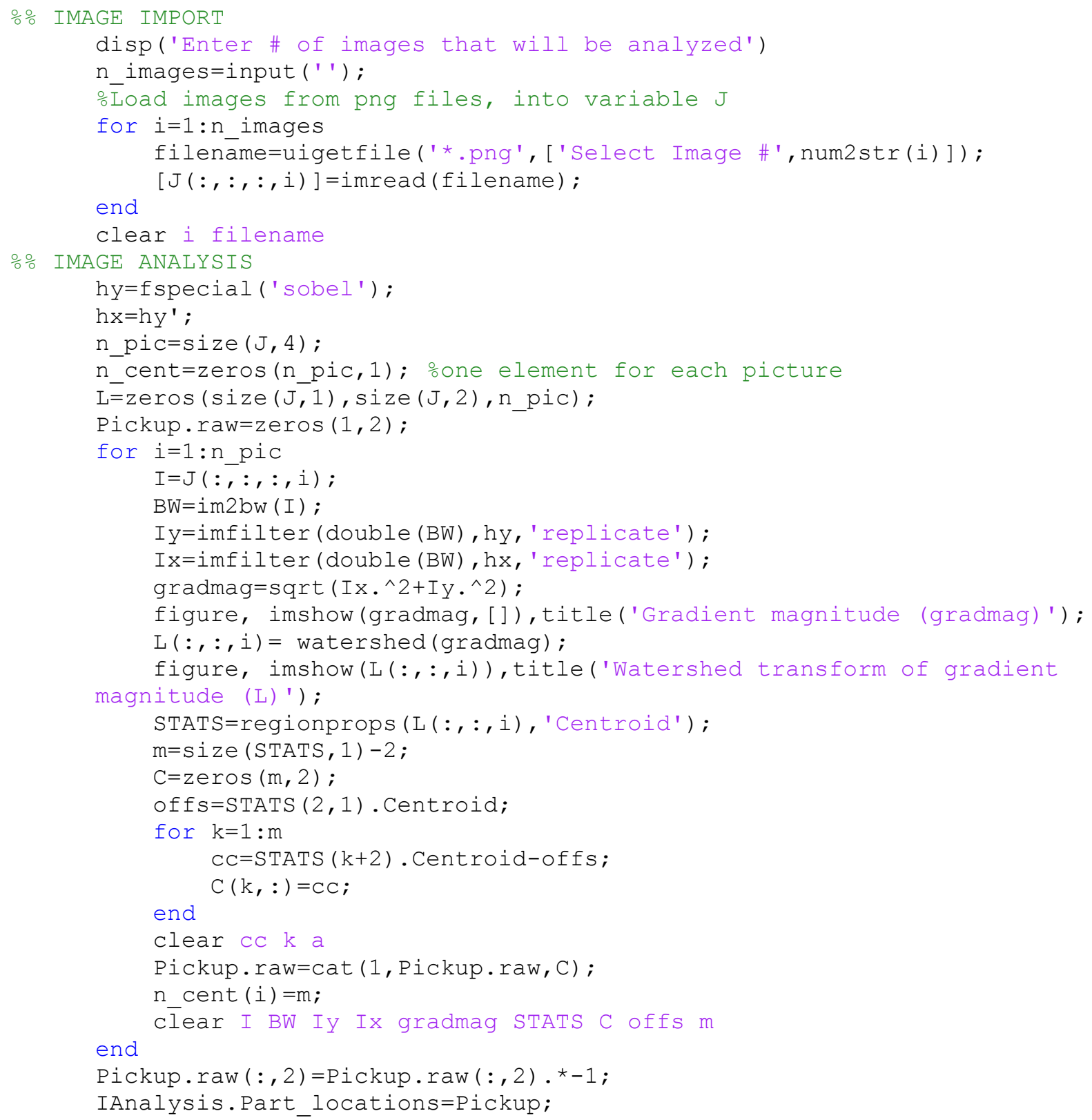


IAnalysis.number_images=n_pic;

IAnalysis. number parts $=$ size $($ Pickup.raw, 1) -1;

IAnalysis.number_parts_per_image=n_cent;

clear L hx hy i cal_roti n_pic n_cent

- Plot centroids for all regions

$\mathrm{x}=\mathrm{Pickup}$. raw $(:, 1)$;

$\mathrm{y}=$ Pickup.raw $(:, 2)$;

figure, plot(x,y,'Marker', '.' ', Linestyle', 'none') ;

title('Pickup Locations, in pixel units');

clear $x$ y

\section{응 MEASUREMENTS PROMPT}

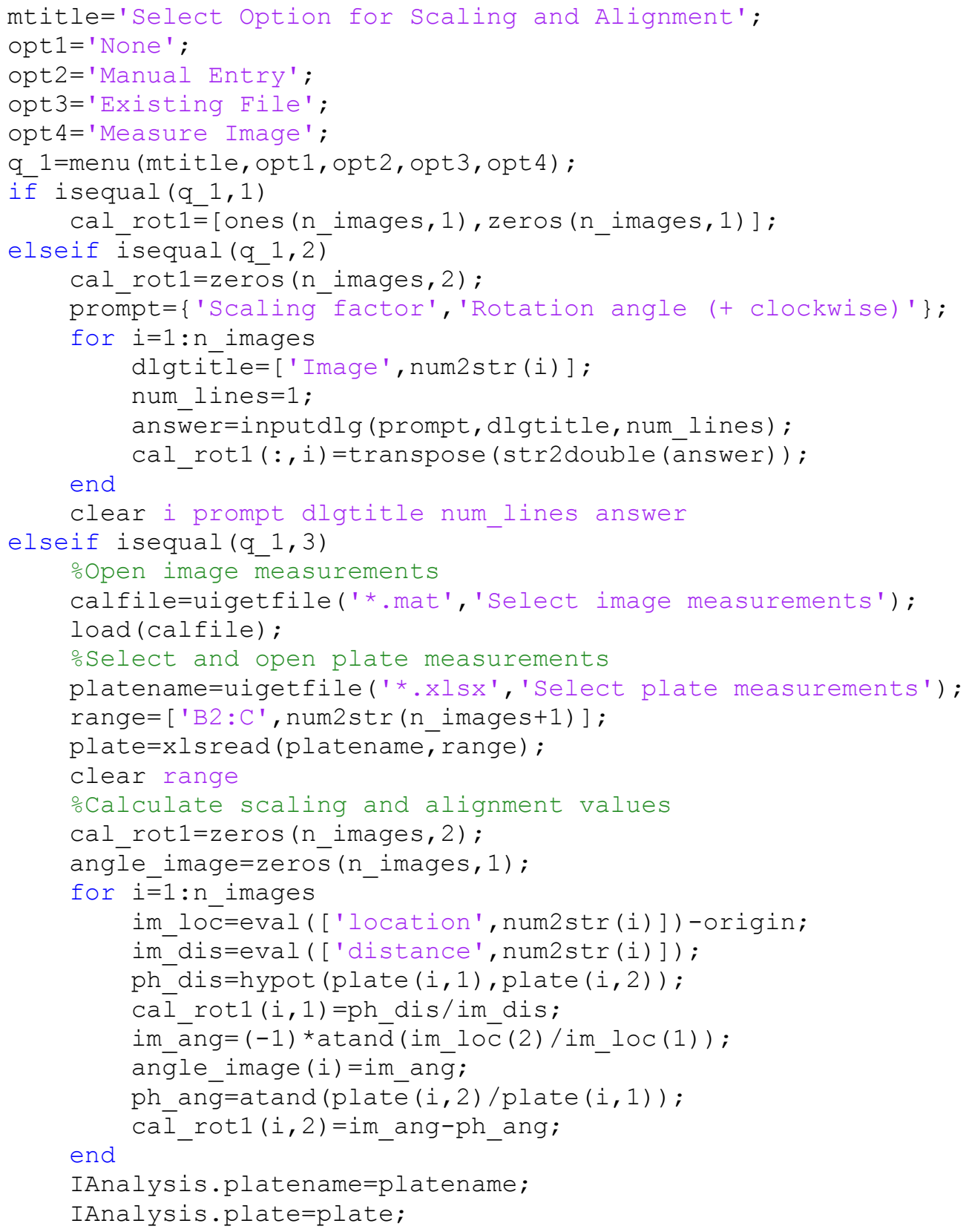




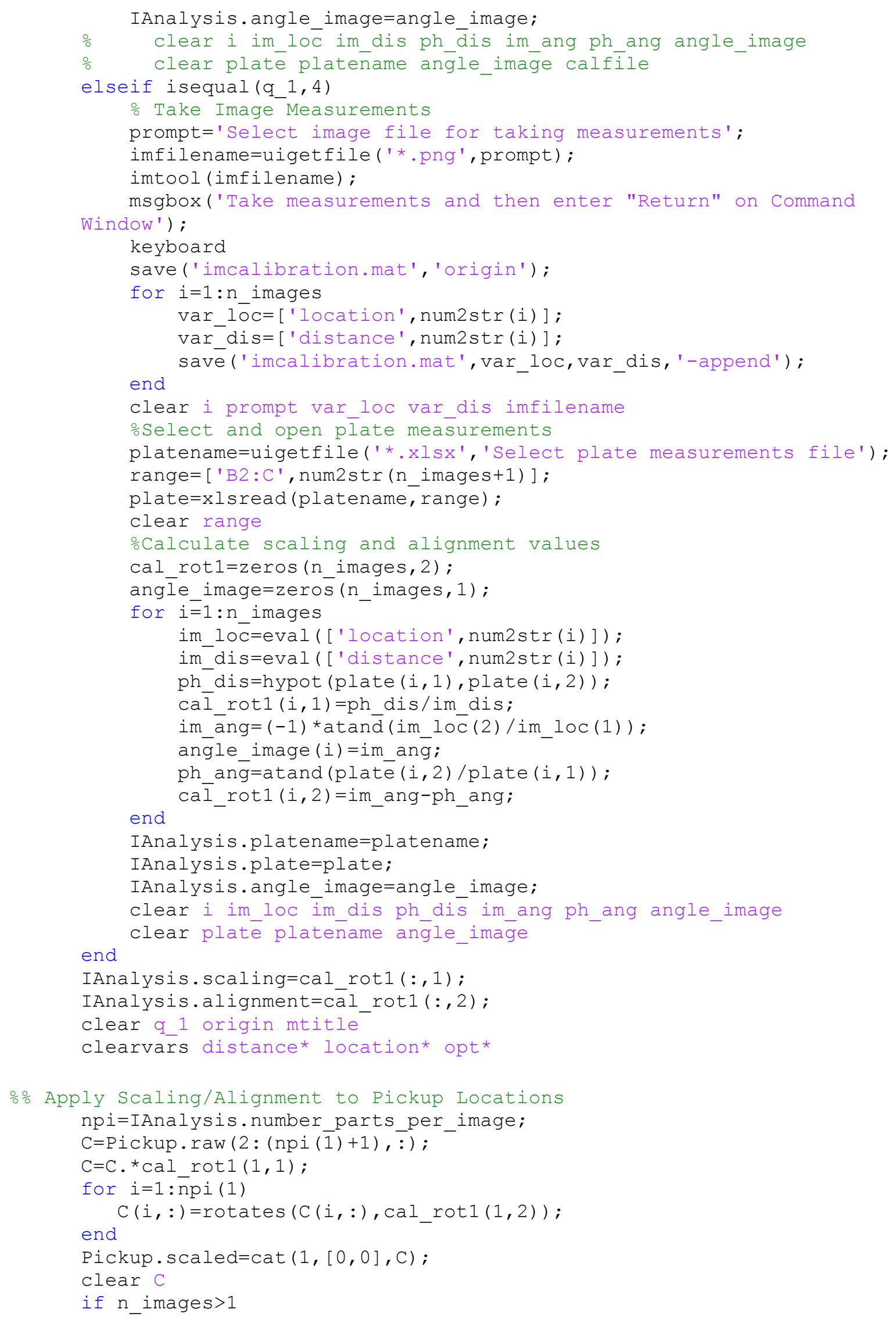




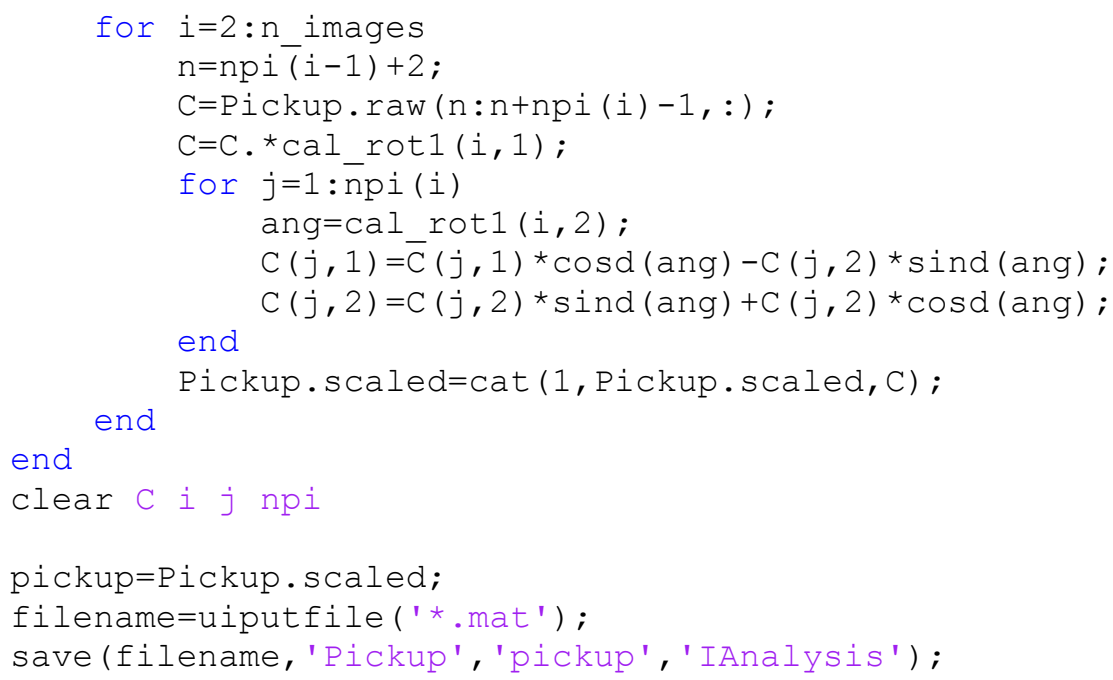

\section{C.2 Matlab Script for Generating GCode Programs that Run the Pick-and-Drop System During SA Tests}

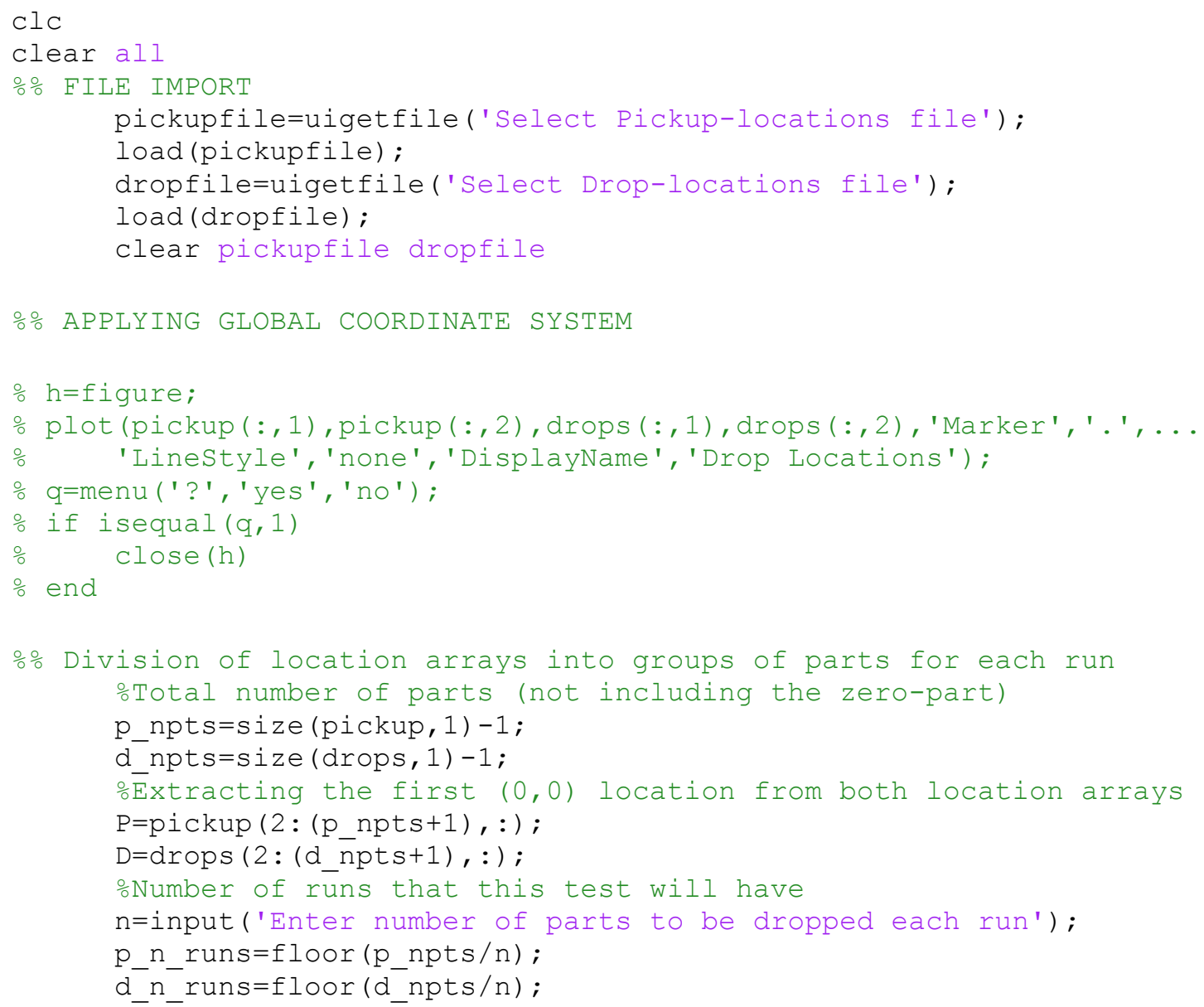




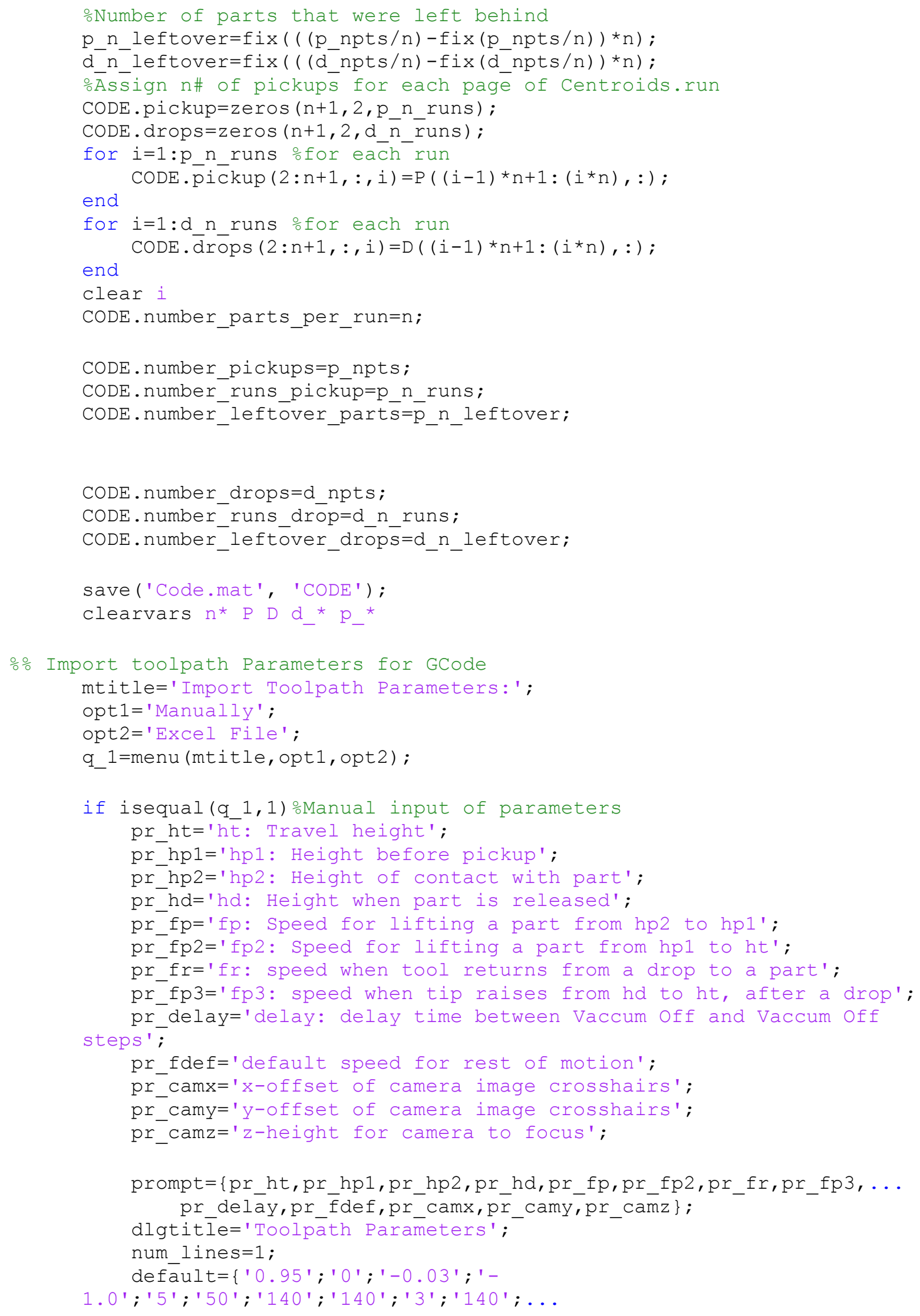




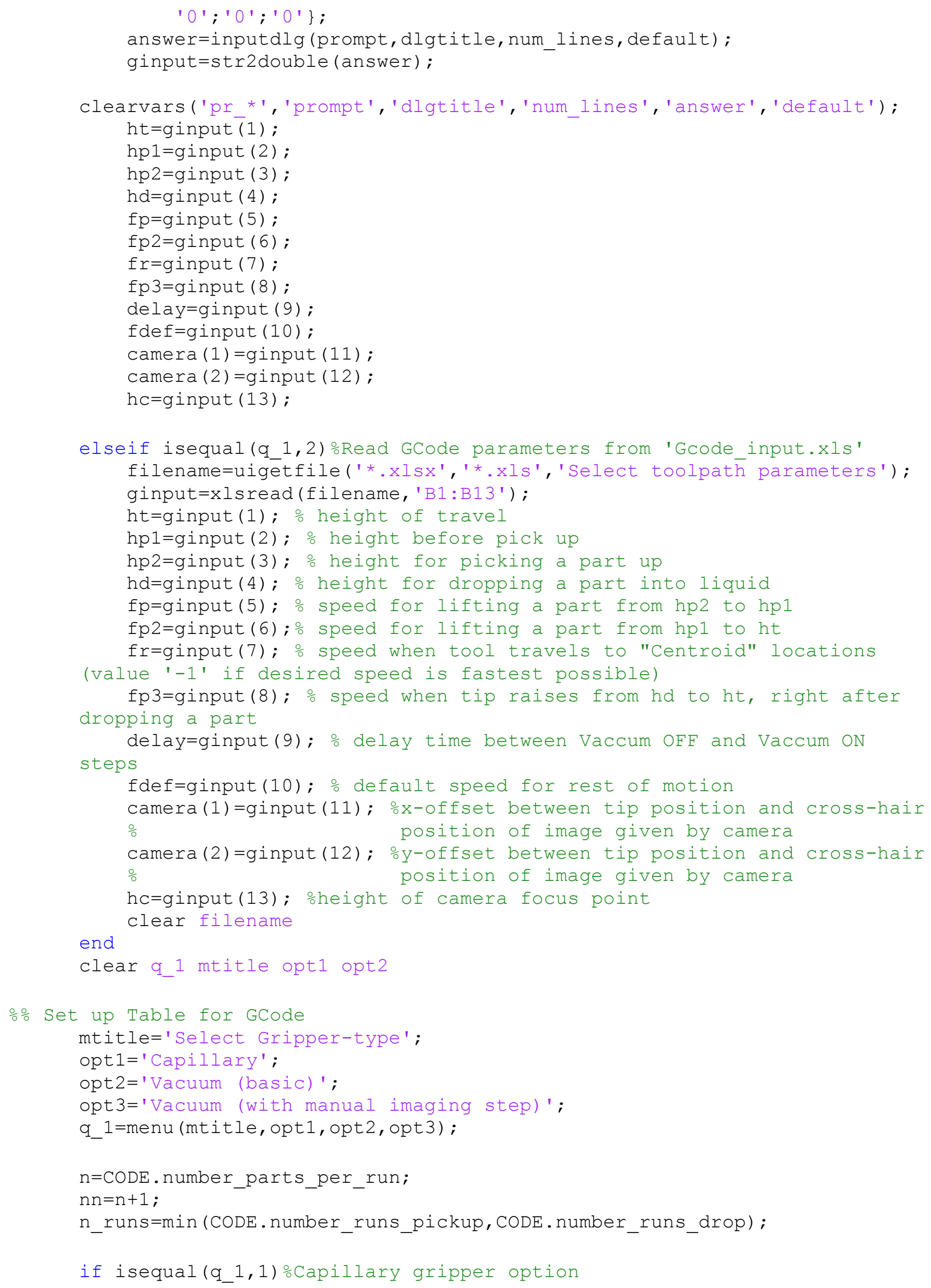




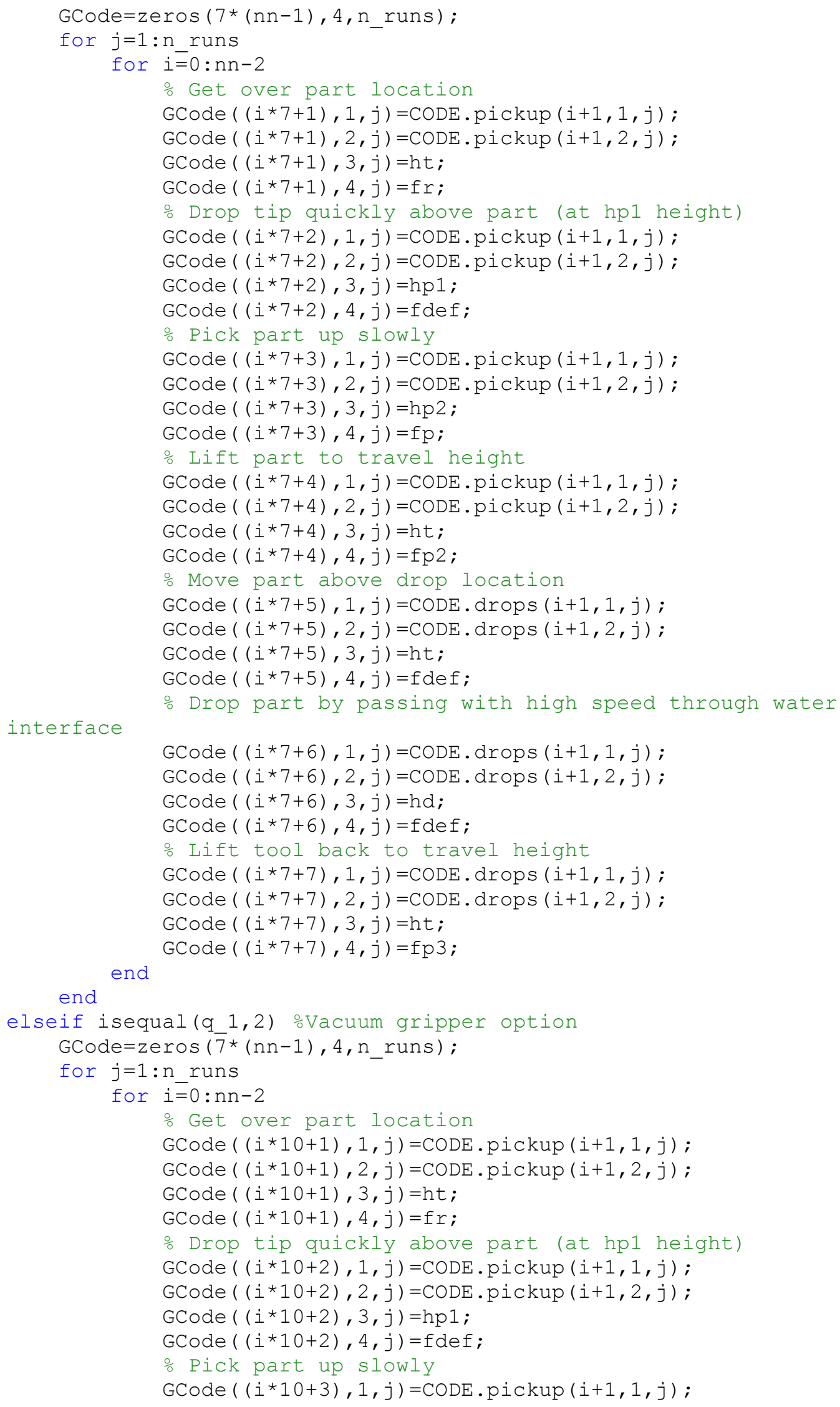




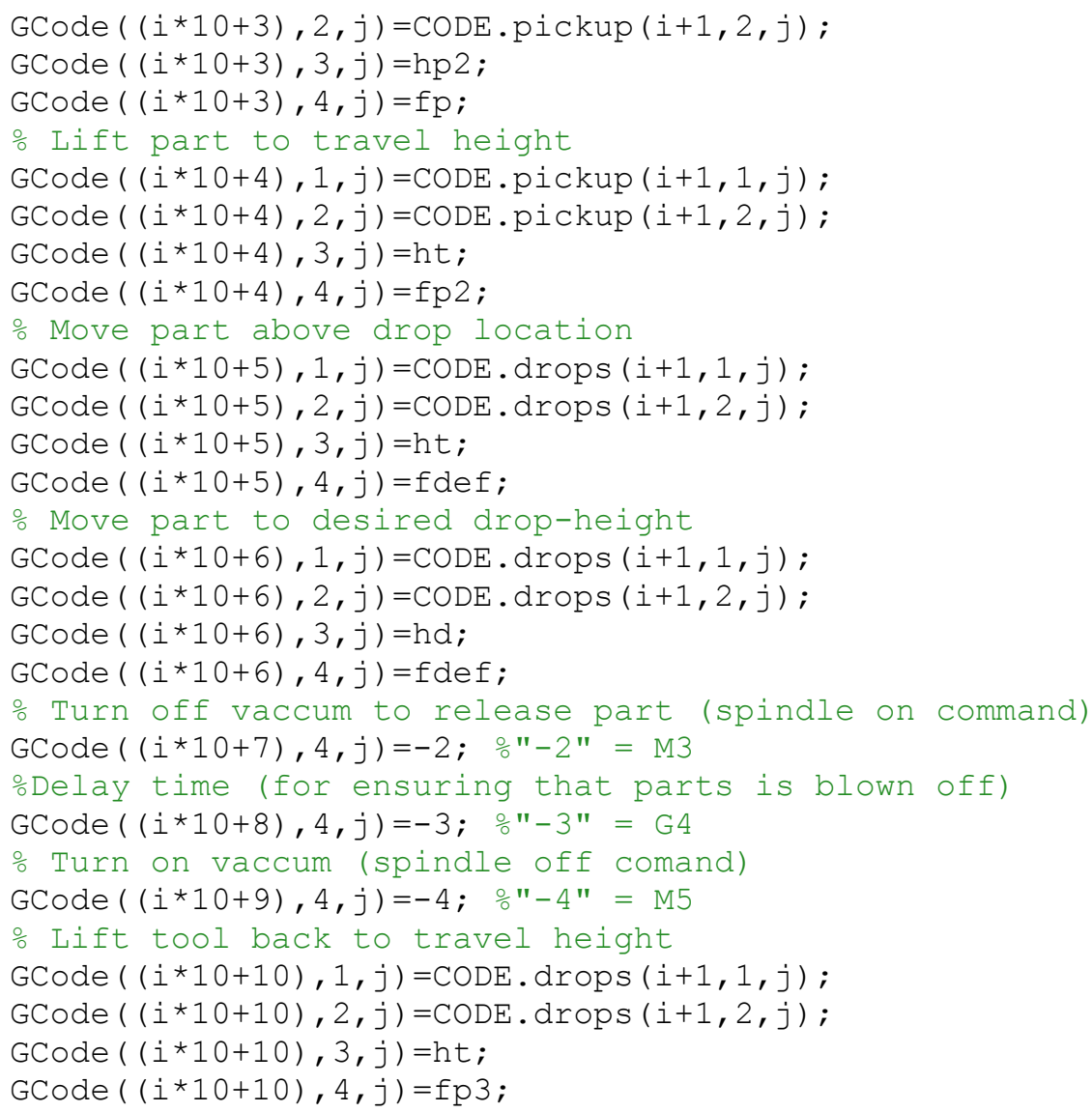




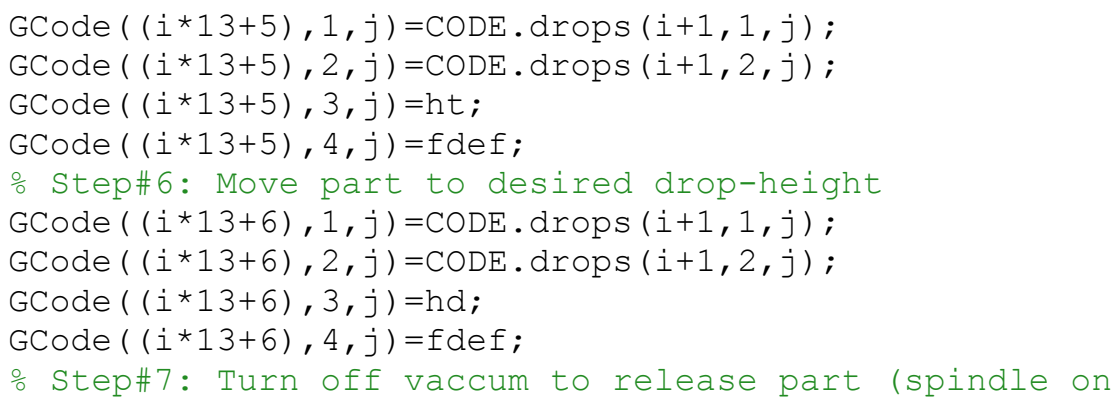




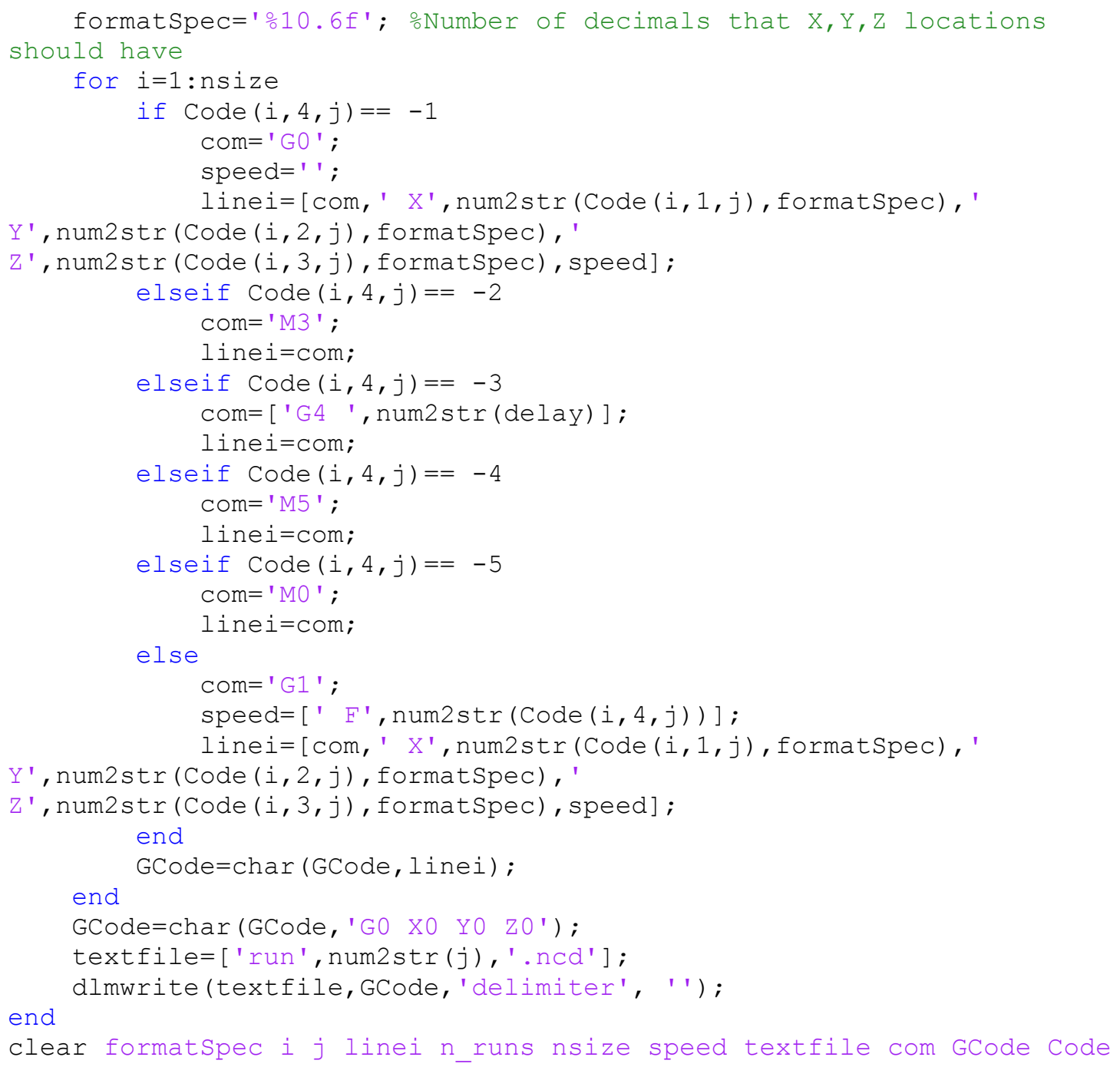




\section{APPENDIX D:}

\section{COPYRIGHT PERMISSION}

The following statement from IEEE permits the reproduction of previously published content (i.e. Figure 1 and Chapter 4 ) in this dissertation.

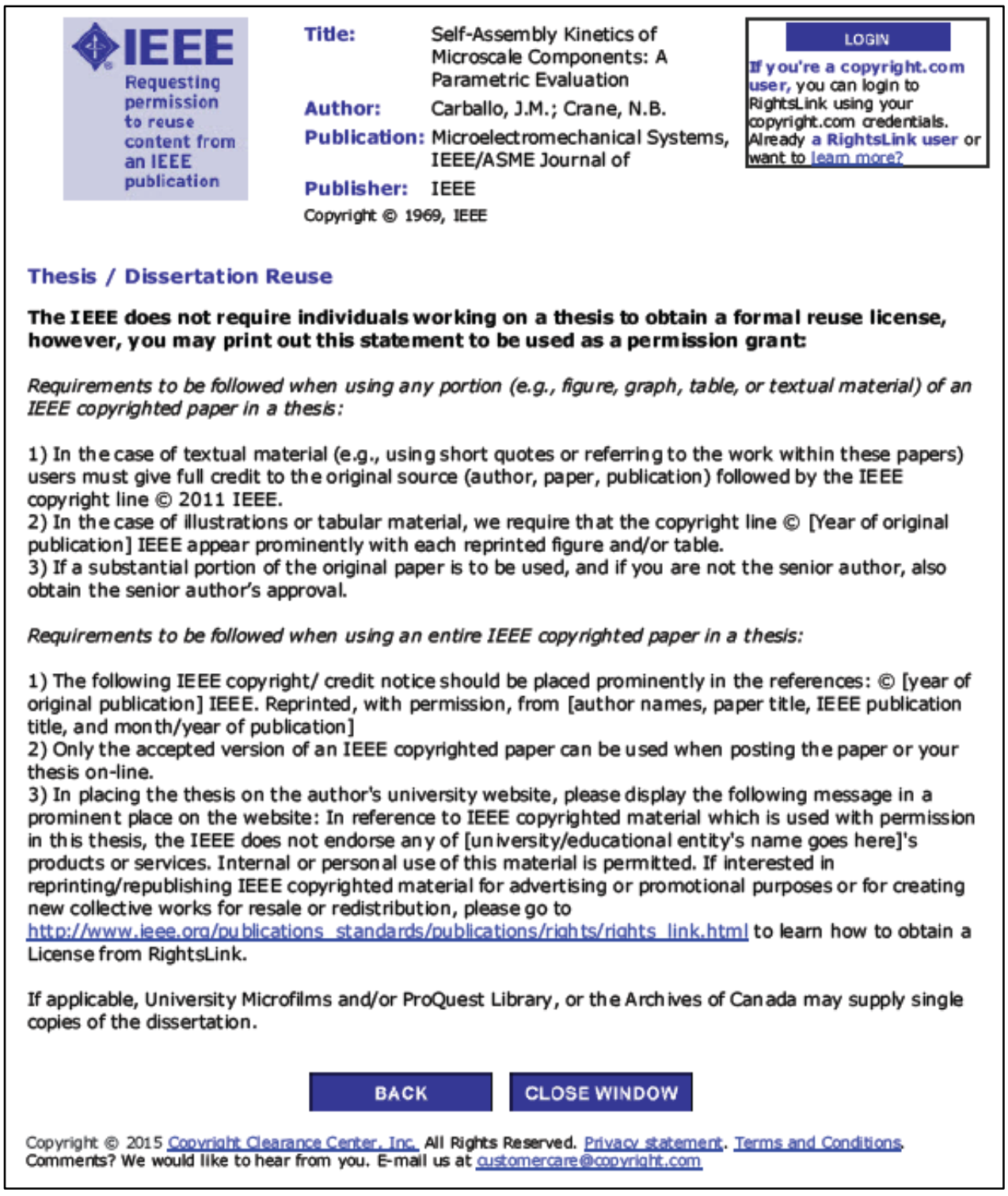




\begin{abstract}
ABOUT THE AUTHOR
Jose graduated from University of South Florida with both a B.S. and an M.S. degree in Mechanical Engineering. During his undergraduate studies, Jose worked as R\&D Co-Op at United Technologies in Farmington, CT. Thereafter and back in Florida, he worked at the USF Thin Films laboratory while pursuing his M.S. degree. Additionally, he worked temporarily for Baxter Healthcare as a Systems Engineering Intern, at Waukegan, IL. After completing his M.S. program, Jose stayed at University of South Florida to pursue his Ph.D. During this time he has been working at the USF Micro-Integration Laboratory and also worked temporarily as an R\&D Intern at Medtronic, Diabetes in Northridge, CA.

Jose currently lives in Florida and seeking to establish a long term career in R\&D where he can leverage his research and industry experience in machine design, microscale fabrication and integration.
\end{abstract}

